\title{
Cancer Associated Fibroblast-Derived IL-6 Determines Unfavorable Prognosis in Cholangiocarcinoma By Affecting Autophagy- Associated Chemoresponse
}

\section{Suyanee Thongchot}

Mahidol University Faculty of Medicine Siriraj Hospital

\section{Chiara Vidoni}

Università del Piemonte Orientale Amedeo Avogadro

Alessandra Ferraresi

Universita' del Piemonte Orientale Amedeo Avogadro

Watcharin Loilome Loilome

Khon Kaen University Faculty of Medicine

Narong Khuntikeo

Khon Kaen University Faculty of Medicine

Prakasit Sa-Ngiamwibool

Khon Kaen University Faculty of Medicine

Attapol Titapun

Khon Kaen University Faculty of Medicine

\section{Ciro Isidoro}

Universita' del Piemonte Orientale Amedeo Avogadro

Nisana Namwat ( $\nabla$ nisana@kku.ac.th )

Khon Kaen University https://orcid.org/0000-0002-6743-5047

\section{Research}

Keywords: cholangiocarcinoma, cancer-associated fibroblasts, interleukin-6, cytokines, autophagy, desmoplastic stroma, cancer therapy

Posted Date: December 2nd, 2020

DOl: https://doi.org/10.21203/rs.3.rs-117710/v1

License: (c) (1) This work is licensed under a Creative Commons Attribution 4.0 International License. Read Full License 


\section{Abstract}

Background: Interleukin-6 (IL-6) massively released by cancer-associated fibroblast (CAFs) has been shown to associate with the malignant behavior of cholangiocarcinoma (CCA). In vitro studies demonstrated the ability of CAFs-derived IL- 6 to inhibit autophagy in CCA cells thus promoting their proliferation and invasiveness potential. Here, we aimed to validate with clinical and molecular data the hypothesis that CAFs infiltration and release of IL- 6 predict poor prognosis in CCA patients following dysregulation of autophagy in cancer cells.

Methods: Stromal IL- 6 and cancer cell-associated autophagy proteins LC3 and p62 were assayed by Tissue MicroArray immunohistochemistry and their expression correlated with overall survival (OS) in a cohort of 70 CCA patients. Additionally, copy number and mRNA expression data of BECN1, MAP1-LC3B, p62/SQSTM1 and IL6 were extracted from a CCA database in TCGA and correlated with OS. 5-FU Cytotoxicity in CCA cells was assessed by cell counting, clonogenic assay, cytofluorometry and western blotting and immunofluorescence of apoptotic-related proteins.

Results: We show that patients bearing a CCA with low production of stromal IL-6 and active autophagy flux in the cancer cells have the best prognosis and this correlates with a more effective response to postoperative chemotherapy. Similar trend was observed in CCA patients from TCGA database. In vitro experiments with primary CAFs isolated from human CCA and epigenetic manipulations showed that IL-6 plays a pivotal role in determining the autophagy-associated apoptotic response to chemotherapeutic drug in cultured human CCA cells.

Conclusions: Our data support a therapeutic strategy that includes autophagy-enhancing drugs along with adjuvants limiting the stromal inflammation (i.e., the secretion of IL-6) to improve the survival of CCA patients.

\section{Background}

Cholangiocarcinoma (CCA), the cancer of bile duct epithelia, is the second most common primary malignancy of the hepato-biliary system, and its incidence rate has significantly increased over the last decades worldwide [1, 2]. CCA remains a major concern especially in the Southeast Asia, where the pathogenesis is essentially associated with chronic liver fluke infection $[3,4]$. Otherwise a deadly disease with a $9 \%$ mortality ratio within three months [5], surgical resection followed by radio- and/or chemotherapies offers survival rates of approximately $20-40 \%$ and $5-10 \%$ at 5 -year and 10 -year, respectively [6, 7]. Surgery and chemotherapy (usually with gemcitabine, cisplatin or 5-FU) elicit however very modest success [8]. Unfortunately, late diagnosis often precludes the possibility of surgical intervention. Additionally, even in this case recurrence and progression with emergence of polychemoresistance are very common, which explain the very poor prognosis of CCA) [9-11].

In the last decades, gene profiling [12-15] and clinical-histopathology [16-20] studies have greatly added to our knowledge of the genes and molecular pathways that are involved in the pathogenesis and 
progression and define the prognosis of CCA. The top genes that were found abnormally expressed in CCA, with a frequency ranging from 10 to $>50 \%$ depending on the topographical location (intra or extrahepatic), the population studied and the method include TP53, KRAS, CDKN2/p16 INK4, FGFR2 gene fusions, $E R B B 2, I D H 1$ and $A R I D 1 A[12,15,21]$. The main molecular pathways that were found altered in CCA include the chromatin rearrangement, epigenetic regulation, proliferation signaling, apoptosis and DNA repair [21-23]. Besides, there are some evidence for involving autophagy in cholangiocarcinogenesis [24-26]. Autophagy, the lysosomal-driven macromolecular degradation pathway playing a major role in tissue homeostasis, is known to be dysregulated in cancer cells subject to changes of the tumor microenvironment in terms of nutrients, oxygen, growth factors, cytokines and other signaling molecules [27-29]. Recently, we have shown that IL-6 secreted by primary Cancer Associated Fibroblasts (CAFs) isolated from human CCA is capable of inhibiting autophagy in CCA cells and by doing so it stimulates their proliferation and invasiveness potential [30]. It remains to determine whether such an effect is indeed happening in CCA bearing patients and, if so, whether it has any prognostic value and significance for therapeutic intervention.

In this work, we address this issue adding clinical evidence and in vitro mechanistic explanations in support of the view that CAFs infiltration indeed causes CCA progression through impairing the autophagy flux in cancer cells thus resulting in reduced chemoresponsiveness. Our experimental data, together with bioinformatic analysis of TCGA data, demonstrate the relevance of IL-6 and of autophagy proteins as prognostic markers in CCA patients. We also show that IL-6 is involved in autophagymediated response to the chemotherapeutic drug 5-FU. The present data have therapeutic implications supporting the inclusion of autophagy-enhancing drugs along with adjuvant therapies capable of dampening stromal inflammation for a better management of CCA.

\section{Methods}

\section{Patients, samples, and Ethical issues}

CCA tissue microarray (CCA-TMAs) of paraffin-embedded cases originated from primary tumors of 70 patients who admitted to surgical wards of Srinagarind Hospital, Khon Kaen University, Khon Kaen, Thailand, collected between 2014-2016. Written informed consent was obtained from all patients in accordance with the Declaration of Helsinki and its later revision. The Human Research Ethics Committee, Khon Kaen University, approved the research protocol (\#HE601063).

\section{Cell lines and primary cancer associated fibroblasts}

The human CCA cell line KKU-213 was obtained from the Liver Fluke and Cholangiocarcinoma Research Center, Khon Kaen University (Thailand). KKU-213 cell line was maintained in standard culture conditions in Ham's F-12 medium supplemented with 10\% heat-inactivated FBS, 1\% Glutamine (Sigma-Aldrich) and $1 \%$ Penicillin/Streptomycin (Sigma-Aldrich) (referred to as CoM, Control Medium), in atmosphere of $5 \%$ $\mathrm{CO}_{2}$ at $37^{\circ} \mathrm{C}$. Primary culture of CCA-associated fibroblasts (CAFs) was isolated using a standard 
procedure from CCA of a patient who underwent surgery at Srinagarind Hospital, Khon Kaen University (Thailand). CAFs were cultivated in Ham's F-12 media containing 10\% FBS for 10-15 days to allow the formation of colonies (designated as passage 0 ). CAFs cells were sub-cultured when $80 \%$ confluent, banked and used for experimental studies at passages $5-13$.

\section{Antibodies and Reagents}

The primary antibodies used for immunohistochemistry (IHC), immunofluorescence (IF) and Western blotting (WB) were purchased from Abcam (Cambridge, MA, USA), anti-LC3 (IHC 1:500; WB 1:1,000; IF 1:100), anti-p62 (IHC 1:500; WB 1:500; IF 1:100), anti-IL-6 (IHC 1:1,000; WB 1:1,000; IF 1:100), BAX (WB 1:2,000; IF 1:100), and BCL-2 (WB 1:1,000; IF 1:100). Peroxidase-conjugated Envision ${ }^{\mathrm{TM}} \mathrm{IHC}$ secondary antibody was purchased from DAKO, Denmark. Horse radish peroxidase (HRP)-conjugated goat antimouse $(1: 2,000)$ or goat anti-rabbit $(1: 2,000)$, both from BioRad (Hercules, CA) were used as secondary antibodies for WB. Alexa Fluor ${ }^{\mathrm{TM}} 555 \mathrm{goal}$ anti-mouse lgG (red fluorescence) from Life technologies or Alexa Fluor ${ }^{\mathrm{TM}} 488 \mathrm{goal}$ anti-rabbit IgG (green fluorescence) from Invitrogen, both from Thermo Fisher Scientific Co. Itd, MA, USA were used for IF. Hoechst, trihydrochloride trihydrate from Invitrogen was added to stain the nucleus.

\section{Cytotoxicity assay}

Cells were plated in triplicate in $96-$ well plates at $1 \times 10^{3}$ cells confluence per well. Twenty-four hours later, the cells were treated with CAFs-CM with or without 5-FU or their combination for $48 \mathrm{~h}$ at the concentrations indicated. Cell viability was measured using a SRB assay. Cell viability numbers were determined by calculating the average OD from three wells and the experiment was repeated in triplicate.

\section{Cell viability}

Cell viability was evaluated using a colorimetric assay. Cell viability was measured based on colonies formation assay stained with $0.5 \%$ crystal violet. Briefly, KKU- 213 cells ( 200 cells/well) were cultured in six-well plates and incubated with 5-FU for 10 days. The medium and substances for treatment were renovated every 3 days (day 3 , day 6 , and day 9 ). At the end of the treatment, the cells were washed with 1X PBS, fixed with $10 \%$ TCA (trichloroacetic acid), stained with a $0.05 \%$ crystal violet solution, and washed with tap water until excess dye was removed. The colony number was counted by photometric measurements using the CellCounter software (Nghia, Ho) version 0.2.1. Three independent experiments were performed for each assay condition. Flow cytometry was performed as previously described using a propidium iodide ( $\mathrm{Pl}, 50 \mathrm{\mu g} / \mathrm{ml}$ final concentration) (Alexis Laboratories, San Diego, CA, USA) and analyzed in a FacScan flow cytometer (Becton Dickinson, USA).

\section{Immunohistochemical analysis}

LC3, p62 and IL- 6 were detected on the CCA-TMAs of 70 paraffin embedded sections using standard immunohistochemistry protocols. In brief, tissue sections were deparaffinized in xylene and rehydrated in 
a series of concentrations of ethanol. Thereafter, antigen retrieval was performed by microwaving sections in $10 \mathrm{mM}$ sodium citrate $(\mathrm{pH} 6)+0.1 \%$ Triton X-100, 10 min for LC3 and p62 protein, and $10 \mathrm{mM}$ sodium citrate $(\mathrm{pH} 6), 10$ min for IL-6 protein antigens retrieval. Then, the sections were endogenous peroxidase activity blocked with $0.3 \% \mathrm{H}_{2} \mathrm{O}_{2}$ for 30 min. Nonspecific binding was blocked by $10 \% \mathrm{skim}$ milk in 1x phosphate-buffered saline (1X PBS) for $30 \mathrm{~min}$. Sections were incubated with the primary antibody at $4^{\circ} \mathrm{C}$ in a moisture chamber, overnight. After that, sections were incubated with peroxidaseconjugated Envision secondary antibody (Dako, Glostrup, Denmark). The color was developed with $0.1 \%$ diaminobenzidine tetrahydrochloride solution for $5 \mathrm{~min}$ and the sections were counterstained with Mayer's hematoxylin. The CCA-TMAs sections were observed under a light microscope (Eclipse, Ni-U, Nikon Instruments Inc. USA) by using the high magnification power $x 200$. The staining frequency of proteins was semiquantitatively scored based on the percentages of positive cells, as follows: $0 \%=$ negative; $1-25 \%=+1 ; 26-50 \%=+2$; and $>50 \%=+3$. The intensity of protein staining was scored as weak $=1$, moderate $=2$, and strong $=3[31]$.

\section{Western blot analysis}

KKU-213 cell line was lysed with radioimmuno-precipitation assay (RIPA) buffer containing protease inhibitor cocktails, 0.5M NaF, 0.2M NaVO 4 , $1 \mathrm{M}$ Tris-HCl pH 7.5, 0.5M EDTA, 2.5M NaCl, 10\% (v/v) NP-40, $10 \%(\mathrm{w} / \mathrm{v}$ ) SDS, Triton X-100 and deionized water. Protein assay using bicinchoninic acid (BCA; Thermo ScientificTM, Rockford, USA). $20 \mu \mathrm{g}$ of liver homogenated was fractionated by SDS-PAGE and transferred to a polyvinylidene fluoride membrane (Whatman, Dassel, Germany). The membranes were incubated overnight at $4^{\circ} \mathrm{C}$ with the primary antibody, followed by incubation with the appropriate secondary antibody at room temperature for $1 \mathrm{~h}$. Enhanced Chemiluminescence Plus solution (GE Healthcare, Buckinghamshire, UK). Band intensity was quantified with ImageQuant Imager and ImageQuant analysis software (GE Healthcare, Uppsala, Sweden). Intensity of the bands was estimated by ImageJ software $(\mathrm{NIH}$, Bethesda, MD, USA). The membranes were also stained for $\beta$-actin as an internal control of protein loading.

\section{Immunofluorescence staining}

CCA cell on coverslips were selected for double immunofluorescence staining with the indicated primary antibody. The appropriate secondary fluorescent antibody and Hoechst were added to stain the nucleus. Antibodies were diluted in $0.1 \%$ Triton X-100 in 1 X PBS $+10 \%$ FBS. Fluorescence was captured with a Carl ZEISS 710 confocal fluorescence microscope (Carl Zeiss Microscopy GmbH, Jena, Germany) equipped with ZEN Software.

\section{Transient knockdown of small interfering RNA for IL-6 into CAFs}

CAFs $\left(1 \times 10^{5}\right.$ cells $)$ isolated from CCA tissues were seeded in a six well plate for $24 \mathrm{~h}$ prior to transfection. Small interfering RNA (si-RNA) transfection was performed using Lipofectamine RNAiMAX (Invitrogen). Stealth RNAi si-RNA for IL-6 (Invitrogen), or stealth RNAi si-RNA negative control (Invitrogen), were transfected at a final concentration of $5 \mathrm{nM}$. After $24 \mathrm{~h}$ of transfection, media was changed to $10 \%$ 
FBS HAM F'12 and cultured for $24 \mathrm{~h}$. CAFs-CM was collected by growing CAFs for 2 days until CAFs reached about $80 \%$ confluence. Then CAFs-CMs were centrifuged at $1,000 \mathrm{~g}$ for $10 \mathrm{~min}$ to remove cell debris, sterile filtered and stored at $-80^{\circ} \mathrm{C}$ until used.

\section{The Cancer Genome Atlas (TCGA)}

We have collected the clinical data on cholangiocarcinomas from cBioportal publicly available online platforms (www.cbioportal.org). The dataset that we interrogated from the Cancer Genomic Atlas Project (TCGA, Firehose Legency) report a total number of 34 cholangiocarcinoma patients/samples (including intrahepatic, extrahepatic and perihilar cancer subtypes). We extracted the information related to messenger RNA (mRNA) expression (at https://genome-cancer.ucsc.edu/proj/site/hgHeatmap), and we analyze the cross-comparison between pair of genes based on mRNA expression groups for BECN1, MAP1-LC3B, p62/SQSTM1 and IL6. All statistical analyses were performed using Excel and SAS software (9.4.version, SAS Institute Inc., Cary, NC) following SAS/STATs and represented in box plot format. The $P$ value $<0.05$ was considered significant.

\section{Statistical analysis}

The relationship between IL-6 and autophagy proteins expression and clinicopathological factors was analyzed using $\chi 2$ test, Fisher's exact test or Kruskal-Wallis test. OS was defined as the period from the date of surgery to the date of death or the last day of follow up. Survival curves were analyzed with the Kaplan-Meier method and compared using the log-rank test. Multivariate analysis of putative prognostic factors was evaluated in a Cox proportional hazards model. Correlation between two continuous variables was calculated using Pearson's correlation coefficient ( $r$ ). Data are presented as mean \pm S.D. All analyses were two-sided $P$-value less than 0.05 was considered statistically significant. Statistical analyses were performed using SPSS 20.0 statistics software (SPSS Inc., Chicago, IL).

\section{Results}

\section{Clinicopathological data of CCA patients}

Seventy cases of CCA tissue micro array (TMA) liver sections, from male (62\%) and female (38\%) patients aged between 32 and 82 years old (median $=60$ years old) were included in the study. Pathological examination confirmed that all cases were intra-hepatic adenocarcinomas from bile ducts. All 70 cases were subjected to surgical resection. Chemotherapy was administered to 6 patients before and to 24 patients after surgery, while 40 patients were not subjected to any chemotherapy. During a median of 1.3 (range, $0.01-5.20$ ) months of follow-up, 34 of the 70 patients (48.57\%) died. Follow-up information were available for the 36 patients surviving up to 100 months. The clinicopathological characteristics of CCA patients, including age, sex, tumor staging, tumor size, tumor-node-metastasis (TMN), histological grading and chemotherapy with respect to the level of LC3 and p62 expression in cancer cells and of IL-6 in CAFs are presented in Table 1. 
Table 1

Clinicopathological variables of and IL-6 in CAFs, LC3 and p62 in cancer cells of 70 CCA samples in the study cohort

\begin{tabular}{|c|c|c|c|c|c|c|c|c|c|c|}
\hline \multirow[t]{2}{*}{ Factor } & \multirow[t]{2}{*}{$n$} & \multicolumn{2}{|l|}{ LC3 } & \multirow[t]{2}{*}{$P$} & \multicolumn{2}{|l|}{ P62 } & \multirow[t]{2}{*}{$P$} & \multicolumn{2}{|c|}{ IL-6 (in CAFs) } & \multirow[t]{2}{*}{$P$} \\
\hline & & Low & High & & Low & High & & Low & High & \\
\hline \multicolumn{11}{|c|}{ Age (years) } \\
\hline$\leq 55$ & 35 & 16 & 19 & 0.316 & 27 & 8 & 0.500 & 20 & 15 & 0.500 \\
\hline$>55$ & 35 & 19 & 16 & & 26 & 9 & & 19 & 16 & \\
\hline \multicolumn{11}{|l|}{ Sex } \\
\hline Female & 27 & 13 & 14 & 0.500 & 22 & 5 & 0.275 & 16 & 11 & 0.412 \\
\hline Male & 43 & 22 & 21 & & 31 & 12 & & 23 & 20 & \\
\hline \multicolumn{11}{|c|}{ Histological types } \\
\hline Non-pap & 33 & 18 & 15 & 0.316 & 26 & 7 & 0.388 & 17 & 16 & 0.335 \\
\hline Papillary & 37 & 17 & 20 & & 27 & 10 & & 22 & 15 & \\
\hline \multicolumn{11}{|c|}{ Tumor staging } \\
\hline I/II & 39 & 17 & 22 & 0.168 & 29 & 10 & 0.496 & 22 & 17 & 0.544 \\
\hline III/IV & 31 & 18 & 13 & & 24 & 9 & & 17 & 14 & \\
\hline \multicolumn{11}{|c|}{ Tumor size (T stage) } \\
\hline $\mathrm{T} 1 / \mathrm{T} 2$ & 40 & 18 & 22 & 0.235 & 32 & 8 & 0.246 & 22 & 18 & 0.542 \\
\hline $\mathrm{T} 3 / \mathrm{T} 4$ & 30 & 17 & 13 & & 8 & 9 & & 17 & 13 & \\
\hline \multicolumn{11}{|c|}{ Metastasis to lymph nodes ( $\mathrm{N}$ stage) } \\
\hline NO & 44 & 23 & 21 & 0.342 & 33 & 11 & 0.099 & 25 & 19 & 0.412 \\
\hline N1/N2 & 26 & 12 & 14 & & 20 & 6 & & 14 & 12 & \\
\hline \multicolumn{11}{|c|}{ Metastasis to organs (M stage) } \\
\hline M0 & 65 & 34 & 31 & 0.178 & 51 & 14 & 0.088 & 37 & 28 & 0.391 \\
\hline M1 & 5 & 1 & 4 & & 2 & 3 & & 2 & 3 & \\
\hline \multicolumn{11}{|c|}{ Resection status } \\
\hline R0 & 49 & 24 & 25 & 0.500 & 37 & 12 & 0.604 & 26 & 23 & 0.339 \\
\hline
\end{tabular}

Correlation between IHC score and clinicopathological by Fisher's exact probability test.

* $p$ value less than 0.05 was considered statistically significant. 


\begin{tabular}{|c|c|c|c|c|c|c|c|c|c|c|}
\hline \multirow[t]{2}{*}{ Factor } & \multirow[t]{2}{*}{$n$} & \multicolumn{2}{|l|}{ LC3 } & \multirow[t]{2}{*}{$P$} & \multicolumn{2}{|l|}{ P62 } & \multirow[t]{2}{*}{$P$} & \multicolumn{2}{|c|}{ IL-6 (in CAFs) } & \multirow[t]{2}{*}{$P$} \\
\hline & & Low & High & & Low & High & & Low & High & \\
\hline R1 & 21 & 11 & 10 & & 16 & 5 & & 13 & 8 & \\
\hline \multicolumn{11}{|c|}{ Drug regimen after surgical resection } \\
\hline No & 46 & 24 & 22 & 0.401 & 38 & 8 & 0.604 & 30 & 16 & $0.025^{\star}$ \\
\hline Yes & 24 & 11 & 13 & & 15 & 9 & & 9 & 15 & \\
\hline \multicolumn{11}{|c|}{ Recurrence after surgical resection } \\
\hline No & 51 & 23 & 28 & 0.141 & 36 & 15 & 0.088 & 29 & 22 & 0.480 \\
\hline Yes & 19 & 12 & 7 & & 17 & 2 & & 10 & 9 & \\
\hline
\end{tabular}

\section{IL-6 expression in fibrotic stroma associates with poor prognosis in CCA patients}

Immunohistochemical (IHC) staining of IL-6 and of the autophagy markers LC3 and p62 was performed and the staining intensity scored in epithelial and in stromal (namely, CAFs) cells. Faintly to negative staining of all the three proteins tested was found in normal bile duct epithelia (Fig. 1a). In contrast, CAFs and cancer cells showed positivity, yet to different extent. On the whole, the percentage of positive cells in human CCA tissues was $50 \%$ for IL-6, $24 \%$ for LC3 and $44 \%$ for p62. The IHC for IL- 6 expression in the cytoplasm was scored separately for epithelial cancer cells and in fibrotic (CAFs-enriched) area (Fig. 1). The staining ranged from absent (score 0 ) to strong (score 3) in CCA tissues. Twenty four of 70 cases (34\%) were positive in only fibrotic area (Fig. 1a-iii), 7 cases (10\%) were positive in cancer epithelial cells (Fig. 1a-iv), and 39 cases (56\%) were positive in both cancer epithelial cells and fibrotic areas (Fig. 1a-v). No significant differences between staining patterns and clinicopathological features, such as age, sex, presence of lymph node metastasis or distant metastasis, grading, as well as tumor location and size were observed (Table 1). To be noted, Fisher's exact test indicated a significant inverse correlation of IL-6 positive staining in cancer cell and fibrotic areas and the drug regimen status (Table $1, P<0.025)$. Further, and most importantly, survival analysis demonstrated that high IL-6 in CAFs-containing fibrotic area was significantly associated with a shorter 5 -year overall survival, in univariate (Fig. $1 \mathrm{c} ; \mathrm{I}=0.024)$ and multivariate analyses (Table $2 ; \mathrm{HR}=0.562 ; \mathrm{Cl}=0.338-0.934 ; P=0.026)$. 
Table 2

Multivariate Cox regression model for disease-free survival including IL-6 in CAFs, LC3 and p62 in cancer cells

\begin{tabular}{|c|c|c|c|c|}
\hline $\begin{array}{l}\text { Variable } \\
\text { (no. of } \\
\text { patients) }\end{array}$ & $\begin{array}{l}\text { No. of patients } \\
\text { who died (5-year } \\
\text { survival cut-off) }\end{array}$ & $\begin{array}{l}\text { Hazard ratio } \\
(\mathrm{HR})\end{array}$ & $\begin{array}{l}\text { 95\% Confidence } \\
\text { interval (Cl) }\end{array}$ & $P$-value \\
\hline \multicolumn{5}{|c|}{ LC3 (in cancer) IHC score } \\
\hline Low & 35 & 1 & & \\
\hline High & 35 & 0.401 & $0.236-0.681$ & $0.001^{\star *}$ \\
\hline \multicolumn{5}{|c|}{ p62 (in cancer) IHC score } \\
\hline Low & 53 & 1 & & \\
\hline High & 17 & 0.699 & $0.400-1.221$ & 0.208 \\
\hline \multicolumn{5}{|c|}{ IL-6 (in CAFs) IHC score } \\
\hline Low & 39 & 1 & & \\
\hline High & 31 & 2.004 & $1.138-3.527$ & $0.016^{*}$ \\
\hline \multicolumn{5}{|l|}{ Age (years) } \\
\hline$\leq 55$ & 35 & 1 & & \\
\hline$>55$ & 35 & 0.505 & $0.308-0.828$ & 0.252 \\
\hline \multicolumn{5}{|l|}{ Sex } \\
\hline Female & 27 & 1 & & \\
\hline Male & 43 & 0.844 & $0.507-1.407$ & 0.516 \\
\hline \multicolumn{5}{|c|}{ Histological types } \\
\hline Non-papillary & 33 & 1 & & \\
\hline Papillary & 37 & 1.174 & $0.725-1.903$ & 0.514 \\
\hline \multicolumn{5}{|c|}{ Tumor staging } \\
\hline I & 11 & 1.336 & $0.380-2.129$ & 0.900 \\
\hline II & 19 & 1.000 & $0.339-0.839$ & 0.511 \\
\hline
\end{tabular}

Multivariate analysis by Cox proportional hazard regression.

Cl 95\% indicates 95\% confidence interval.

${ }^{*} p$ value less than 0.05 was considered statistically significant. 


\begin{tabular}{|lllll|}
\hline $\begin{array}{l}\text { Variable } \\
\text { (no. of } \\
\text { patients) }\end{array}$ & $\begin{array}{l}\text { No. of patients } \\
\text { who died (5-year } \\
\text { survival cut-off) }\end{array}$ & $\begin{array}{l}\text { Hazard ratio } \\
\text { (HR) }\end{array}$ & $\begin{array}{l}\text { 95\% Confidence } \\
\text { interval (Cl) }\end{array}$ & P-value \\
\hline III & 19 & 4.670 & $1.698-12.33$ & 0.414 \\
\hline IV & 21 & 2.070 & $0.330-0.869$ & $0.013^{*}$ \\
\hline Multivariate analysis by Cox proportional hazard regression. & \\
\hline Cl 95\% indicates $95 \%$ confidence interval. & & \\
\hline * $p$ value less than 0.05 was considered statistically significant. & \\
\hline
\end{tabular}

High expression of LC3 along with low expression of p62 in CCA cells correlate with better prognosis

Given the potential involvement of autophagy in CCA progression [24-26, 32-34], we sought to assess the IHC expression profile of the autophagy proteins LC3 (a marker of autophagosome) and p62/SQSTM1 (a marker of the autophagy cargo) in CCA TMAs (Fig. 2). The high IHC score of LC3 (Fig. 2a) showed a positive association with longer overall survival of CCA patients, in univariate (Fig. 2b; green line; $P=0.001$ ) and multivariate analysis (Table $2 ; \mathrm{HR}=0.401 ; \mathrm{Cl}=0.236-0.681 ; P=0.001$ ). Of note, no significant associations between p62 IHC staining and clinical outcome was found (Fig. 2c). Consistently, the prognosis was better in patients bearing a CCA with high expression of LC3 along with low (green line) or high (violet line) p62 expression, compared to patients bearing a CCA with low expression of LC3 (Fig. 2d). The Spearman's correlation test was performed to examine the relationship between LC3 and p62 expressions in epithelial cancer cells (Fig. 2e). The pattern of high LC3 but low p62 showed a positive correlation in CCA tissues (Fig. 2e; rho $=0.518 ; P=0.000$ ), supporting the view that high LC3 was reflecting effective autophagy degradation of the cargo. Remarkably, the combined pattern of high LC3 and low p62 showed a significant correlation with the best overall survival, in univariate (Fig. 2d; green line; $P=0.001)$ and multivariate analysis $(\mathrm{HR}=2.344 ; \mathrm{Cl}=1.222-4.496 ; P=0.01)$.

\section{Correlation between IL-6, LC3 and p62 expression and with clinicopathologic features of CCA patients}

At this point, it was mandatory to check whether inflammation (marked as IL-6 production in fibrotic stroma) and autophagy (marked as LC3 up-expression and p62 down-expression in epithelial cancer cells) were correlated and how the various combinations would correlate with clinical prognosis. With regard to the IHC protein expression scores, of the 70 cases, 18 cases (25.7\%) were classified as low IL-6, low LC3 and low p62 (L/L/L); 11 cases (15.7\%) were classified as low IL-6, high LC3 and low p62 (L/H/L); 1 case $(1.4 \%)$ was classified as low IL-6, low LC3 and high p62 (L/L/H); 9 cases (12.9\%) were classified as low IL-6, high LC3 and low p62 (L/H/H); whereas 14 cases $(20.0 \%)$ were classified as high IL-6, low LC3 and low p62 (H/L/L); 9 cases (12.9\%) were classified as high IL-6, high LC3 and low p62 (H/H/L); 2 cases (2.9\%) were classified as high IL-6, low LC3 and high p62 (H/L/H); and, finally, 6 cases $(8.6 \%)$ were classified as high IL-6, high LC3 and high p62 $(\mathrm{H} / \mathrm{H} / \mathrm{H})$. Assuming the autophagy flux proceeds to 
completion when LC3 is up-expressed along with p62 down-expressed, the autophagy flux was clearly effective in 20 cases, of which 11 presented with low and 9 presented with high expression of IL-6. These numbers do not allow to draw any convincing correlation between the level of IL- 6 in the stroma and the level of autophagy in cancer cells.

Next, we calculated the overall survival for the patients classified according to the above combinations. The Kaplan-Meier curves are shown in Fig. 3. It was found that the pattern of $L / H / L$, representing a low inflammatory stroma (low IL-6 staining) and an efficient autophagy flux in cancer cells (high LC3 and low p62) was significantly associated with the best prognostic clinical outcome (Fig. 3, green line; $P=0.007$ ). In multivariate analysis, this scoring pattern was an independent and significant variable that predicted a favorable prognosis. The hazard ratio [HR] for death based on this variable was 2.535 (95\% confidence interval [Cl] 1.122-5.727; $P=0.025$, Table 3). To be noted, the pattern with low IL-6 in the stroma and high LC3/high p62 in cancer cells (violet line; L/H/H; HR: 1.659, 95\% Cl: 0.813-3.384) also showed a good prognosis when compared to the other patterns having high IL-6 and/or low LC3 expression. Thus, disregarding the expression of p62, the combination of low (stromal) IL-6 with high (cancer) LC3 seems to provide the patients with a higher survival probability (HR: $0.317,95 \% \mathrm{Cl}: 0.147-0.687 \mathrm{for} \mathrm{L} / \mathrm{H} / \mathrm{L}$ and HR: $1.659,95 \% \mathrm{Cl}: 0.813-3.384$ for $\mathrm{L} / \mathrm{H} / \mathrm{H}$; see also Table 3 ), consistent with the data shown in Fig. $2 \mathrm{~d}$. 
Table 3

Multivariate Cox regression model for disease-free survival including IL-6 in CAFs, LC3 and p62 in cancer cells (combined model)

\begin{tabular}{|c|c|c|c|c|}
\hline $\begin{array}{l}\text { Variable } \\
\text { (no. of patients) } \\
\text { (IL-6/LC3/p62) }\end{array}$ & $\begin{array}{l}\text { No. of patients } \\
\text { who died (5-year } \\
\text { survival cut-off) }\end{array}$ & $\begin{array}{l}\text { Hazard ratio } \\
(\mathrm{HR})\end{array}$ & $\begin{array}{l}\text { 95\% Confidence } \\
\text { interval (Cl) }\end{array}$ & $P$-value \\
\hline \multicolumn{5}{|l|}{ Low/Low/Low } \\
\hline No & 52 & 1 & & \\
\hline Yes & 18 & 0.577 & $0.312-1.069$ & 0.081 \\
\hline \multicolumn{5}{|l|}{ Low/High/Low } \\
\hline No & 59 & 1 & & \\
\hline Yes & 11 & 0.317 & $0.147-0.687$ & $0.004^{\star *}$ \\
\hline \multicolumn{5}{|l|}{ Low/Low/High } \\
\hline No & 69 & 1 & & \\
\hline Yes & 1 & 0.322 & $0.043-2.405$ & 0.269 \\
\hline \multicolumn{5}{|l|}{ Low/High/High } \\
\hline No & 61 & 1 & & \\
\hline Yes & 9 & 1.659 & $0.813-3.384$ & 0.164 \\
\hline \multicolumn{5}{|l|}{ High/Low/Low } \\
\hline No & 56 & 1 & & \\
\hline Yes & 14 & 1.901 & $1.028-3.513$ & $0.040^{*}$ \\
\hline \multicolumn{5}{|l|}{ High /High/Low } \\
\hline No & 61 & 1 & & \\
\hline Yes & 9 & 0.612 & $0.298-1.256$ & 0.181 \\
\hline \multicolumn{5}{|l|}{ High /Low/High } \\
\hline No & 68 & 1 & & \\
\hline Yes & 2 & 0.944 & $0.229-3.889$ & 0.937 \\
\hline
\end{tabular}

Multivariate analysis by Cox proportional hazard regression.

Cl 95\% indicates $95 \%$ confidence interval.

${ }^{*} p$ value less than 0.05 was considered statistically significant. 


\begin{tabular}{|lllll|}
\hline $\begin{array}{l}\text { Variable } \\
\text { (no. of patients) } \\
\text { (IL-6/LC3/p62) }\end{array}$ & $\begin{array}{l}\text { No. of patients } \\
\text { who died (5-year } \\
\text { survival cut-off) }\end{array}$ & $\begin{array}{l}\text { Hazard ratio } \\
\text { (HR) }\end{array}$ & $\begin{array}{l}\text { 95\% Confidence } \\
\text { interval (Cl) }\end{array}$ & P-value \\
\hline High /High/High & & & & \\
\hline No & 64 & 1 & $0.460-2.478$ & 0.880 \\
\hline Yes & 6 & 1.067 & \\
\hline Multivariate analysis by Cox proportional hazard regression. & \\
\hline Cl 95\% indicates 95\% confidence interval. & & \\
\hline$* p$ value less than 0.05 was considered statistically significant. & \\
\hline
\end{tabular}

The role of adjuvant chemotherapy and of inflammatory and autophagy markers expression in patient survival

Next, we assessed how and whether postoperative chemotherapy had affected the patient survival depending on the inflammatory and autophagy levels in the cancer. Thirty patients received chemotherapy ( 6 before and 24 after surgery), which included gemcitabine for 8 patients $(26.7 \% ; 12.29 \%$ of total), cisplatin for 7 patients $(23.3 \% ; 10.61 \%$ of total) and 5 -FU for 15 patients $(50 \% ; 21.33 \%$ of total). In an attempt to clarify the respective role of chemotherapy versus CCA inflammation/autophagy status in the clinical outcome, we estimated the overall survival in the patients with favorable status (based on above analysis), i.e., with low IL-6, high LC3 and low p62, that were subjected or not to chemotherapy (see Fig. S1). Additionally, to see whether the chemotherapy per se affected the clinical outcome, we have estimated the overall survival also for the other patients. The Kaplan-Meier curves are shown in Fig. 4. It is clearly apparent that patients who could not benefit of chemotherapy had the poorest outcome (Fig. 4a). Strikingly, the patients bearing a CCA with low IL-6 in fibroblasts and high LC3 and low p62 pattern in cancer cells $(\mathrm{L} / \mathrm{H} / \mathrm{L})$ were the ones most benefiting the adjuvant chemotherapy. This outcome was statistically significant (Fig. 4a, purple line; L/H/L; $P<0.01$ ). The univariate and multivariate analyses were performed to evaluate the prognostic factors affecting overall survival for the low IL-6, high LC3 and low p62 pattern with drug-based therapy (Table 4). Multivariate analysis indicated that the status of drugtreated plus low IL-6 plus high LC3 plus low p62 was an independent factor associated with longer overall survival. Overall, we found a positive correlation between drug-treated and low IL-6 (in fibroblasts) plus high LC3 plus low p62 in cancer cells (Table 5; Pearson $r=0.898, P<0.01$ ). Based on the previous observations, we assumed that high expression of LC3 could impact the chemoresponsivity regardless of the level of p62 expression 
Table 4

Multivariate Cox regression model for disease-free survival including drug receivable, IL-6 in CAFs, LC3 and p62 in cancer cells (combined model)

\begin{tabular}{|llll|}
\hline $\begin{array}{l}\text { Variable } \\
\text { Drug regimen/IL-6/LC3/p62 }\end{array}$ & $\begin{array}{l}\text { Hazard ratio } \\
\mathbf{( H R})\end{array}$ & $\begin{array}{l}\text { 95\% Confidence } \\
\text { interval (Cl) }\end{array}$ & P-value \\
\hline No/Others & 1 & $0.082-4.300$ & 0.607 \\
\hline Yes/Others & 0.595 & & \\
\hline No/Low/High/Low & 1 & $0.019-1.267$ & 0.082 \\
\hline & 0.154 & & \\
\hline Yes/ Low/High/Low & 1 & $0.080-4.227$ & 0.592 \\
\hline & 0.581 & & \\
\hline Multivariate analysis by Cox proportional hazard regression. & \\
\hline Cl 95\% indicates 95\% confidence interval. & \\
\hline * $p$ value less than 0.05 was considered statistically significant. & \\
\hline
\end{tabular}

Table 5

Pearson correlation coefficients between IHC scores of t drug receivable, IL-6 in CAFs, LC3 and p62 in cancer cells (combined model) components in human CCA tissues

\begin{tabular}{|lll|}
\hline & Drug regimen & Low IL-6/High LC3/Low p62 \\
\hline Drug regimen Pearson Correlation & 1 & $.898^{* \star}$ \\
Sig. (2-tailed) & 70 & .000 \\
$N$ & & 70 \\
\hline Low IL-6/High LC3/Low p62 & $.898^{* *}$ & 1 \\
Pearson Correlation & .000 & 70 \\
Sig. (2-tailed) & 70 & \\
$N$ & & \\
\hline$* p$ value less than 0.05 was considered statistically significant. \\
\hline
\end{tabular}

To test this hypothesis, we have estimated the OS in the cohort of 30 patients subjected to chemotherapy considering the group bearing a high LC3-expressing CCA along with low IL-6 in CAFs (regardless of p62 expression) $(n=13) v s$ the other combinations $(n=17)$. Again, the former group showed a better OS 
(Fig. 4b). Finally, we compared the OS of this group (13 patients) $v s$ the group of patients bearing a tumor with high stromal IL- 6 and low cancer cell LC3 $(n=7)$ and the group of patients bearing a tumor with other combinations $(n=10)$, and again the former group showed a better survival (Fig. 4c).

\section{Expression of autophagy markers and clinical outcome in CCA-bearing patients from TCGA database}

Above data refer to a cohort of patients living in a specific geographic area (province of North-Eastern part in Thailand) where liver fluke $O$. viverrini infection is a recognized cause of CCA. To see whether our observation could be extended to CCA cases from other countries, and likely with a different pathogenesis, we have interrogated the TCGA database. Thirty-four cases are reported in the database for which are available, with some exceptions, data on the mRNA expression and Copy Number Variation (CNV) of IL-6 and of the autophagy genes LC3, p62/SQSTM1, and BECN1, along with information on Overall Survival (OS). BECN1 is the first identified mammalian autophagy gene, and it is a haploinsufficient tumor suppressor coding for the BECLIN1 protein [35]. Details of this cohort of patients are provided in Table 1S. The oncoprint showing the alterations in BECN1 and MAP-LC3B gene expression is shown in Fig. S2a. Data on mRNA expression of $L C 3$ were available for 33 CCA, of which 4 with high expression (2 patients underwent chemotherapy) and 29 with low expression (only 7 patients underwent chemotherapy). Though not statistically significant because of the small numbers, the trend shows that the patients bearing a CCA highly expressing LC3 have a better prognosis seen as OS (Fig. 5, panels $a$ and $b$ ). Consistently, better prognosis was observed in patients bearing CCA with MAP-LC3B gene amplification $(n=5)$ compared to patients bearing CCA with diploid CNV $(n=22)$ or with shallow (monoallelic) deletion $(n=6)$ (not shown). Data on BECN1 mRNA expression was available for 34 patients (Fig. S2b). Quite surprisingly, the 29 patients bearing a CCA expressing low level of BECN1 showed a better OS (not significant; $P=0.23$ ) than the 5 patients bearing a CCA expressing high level of $B E C N 1$ (Fig. S2c). It should be considered, however, that the latter patients were not subjected to chemotherapy whereas in the group of low BECN1 expressors 9 patients were subjected to chemotherapy. Also, to be noted, the 5 CCA with high BECN1 expressed low level of LC3 (Fig. S2d). Interestingly, 4 patients with low BECN1 and high $L C 3$ tumor (2 of them underwent chemotherapy and 2 did not) showed a much better prognosis (not significant; $P=0.37$ ) than 5 patients with high BECN1 and low $L C 3$ tumor (Fig. S2e). Data on p62/SQSTM1 gene alteration and OS were available for 33 patients (Fig. S3). Though not statistically significant $(P=0.99)$ because of the small numbers, we found that 29 patients bearing CCA with low level of $p 62$ (only 8 underwent chemotherapy) showed a much better prognosis than 4 patients bearing CCA with high level of $p 62$ (no one underwent chemotherapy) (Fig. S3). Assuming that low accumulation of p62 in cancer cells is indicative of increased autophagy flux, these data are consistent with the above data on LC3. Data on $I L-6$ gene alteration and OS were available for 34 patients (Fig. S4), of which the three bearing CCA with high expression showed the worse prognosis (not significant; $P=0.71$ ). Unfortunately, there are no data available in the TCGA dataset for the level of IL-6 specifically expressed in the stroma.

\section{IL- 6 secreted by CAFs inhibits autophagy and reduces the chemosensitivity of CCA cells}


To explain the above data, we hypothesized that IL- 6 released by CAFs negatively affected the chemosensitivity of neighboring CCA cells via inhibiting the autophagy stress-response to the drug. To this end, we have specifically inhibited the production of IL-6 by transfecting the CAFs with an appropriate si-RNA. The data shown in Fig. 6 prove that the si-RNA transfection effectively down-regulated the expression and secretion in the medium of IL- 6 by CAFs, without altering their myofibroblast-like phenotype (as monitored by a-SMA expression).

Next, we tested whether and how the conditioned media from control or si-RNA-transfected CAFs would affect the autophagy regulation and the chemosensitivity of CCA cells. To this end, we employed the KKU213 cell line that was shown to be very aggressive in previous study [30]. As a representative of chemotherapeutics, we chose 5-FU because it is the one mostly used for the adjuvant therapy in our cohort of patients. We monitored the cell growth of KKU-213 cells incubated for up to $96 \mathrm{~h}$ in medium from control or si-IL-6-transfected CAFs and exposed to 5-FU. SRB staining, which reflects the protein mass in the culture, indicated that cell growth was stimulated by CAFs-conditioned (scramble) medium while it was inhibited by conditioned medium derived from si-IL6-transfected CAFs (Fig. 7a). More importantly, the growth was greatly inhibited by $5-\mathrm{FU}$, and even more when the treatment was performed in the cells incubated with the CAFs-conditioned medium lacking IL-6 (Fig. 7a). The clonogenic assay confirmed that 5-FU could inhibit the proliferation of KKU-213 cells more effectively when incubated in the medium of CAFs avoid of IL-6 (Fig. 7b and 7d). Cytofluorometer analysis further proved that this effect was not merely due to a block of cell proliferation and instead was due to induction of cell death, very likely apoptosis based on the hypodiploid subG1 peak (Fig. 7c and 7e). To be noted, when treated with 5FU the percentage of the subG1 population (referable to apoptotic cells) in control medium was of approx. 33\% while in CAFs-derived medium was of approx. 23\% (i.e., one-third less) and in IL-6 deficient CAFs-derived medium was of approx. $57 \%$ (i.e., it nearly doubled). Finally, we asked whether the CAFsderived effects on chemo-responsivity to 5-FU was linked to autophagy in CCA cells. We further investigated on the link between the CAFs secretions (from scramble si-RNA- or siRNA IL-6-transfected) and the autophagy-dependent response to 5-FU. To this end, we looked at the expression of the autophagy proteins LC3 and p62, of the pro-apoptotic protein BAX and of the ant-apoptotic protein BCL-2. In drug-untreated cultures, compared to the protein expression in cells cultivated in standard medium, the IL-6 rich conditioned CAFs-medium limited the activation of autophagy (reduced conversion of LC3-I into LC3-II; accumulation of p62) and reduced the expression of BAX, whereas the IL-6 deficient CAFs medium (upon si-IL-6 transfection) greatly stimulated the autophagy flux, reduced the expression of BCL-2 and restored the expression of BAX (Fig. 8a-b). On treatment with 5-FU, autophagy was stimulated along with increased expression of BAX in the cells cultivated in standard medium, consistent with induction of a toxic stress response and onset of apoptosis (Fig. 8a-b). Remarkably, this response to 5-FU was largely impaired in the cells incubated with IL-6-rich CAFs medium (from scramble siRNA transfected) while it was enhanced when the cells were treated in the CAFs medium lacking IL-6 (Fig. 8a-b). To definitively assess the functional link between autophagy and apoptosis in response to 5-FU we performed a doubleimmunostaining for LC3 (to mark the cells with ongoing autophagy) and for BAX (to mark the cells undergoing apoptosis). The images in Fig. 8c show that 5-FU can induce BAX-mediated apoptosis only in 
the cells cultivated in standard medium or in IL-6 deficient CAFs-medium while it is not effective in cells cultivated in IL-6-rich CAFs-medium. The double-staining also demonstrates that apoptosis (BAX-positive) ensued in the same cells in which autophagy (LC3-positive) was induced (Fig. 8c).

\section{Discussion}

CCA is a prototype of inflammatory cancer with a highly desmoplastic stroma particularly rich of activated CAFs with myofibroblast-like phenotype [36, 37]. Fibroblasts isolated from CCA tissues have been shown to release a range of soluble factors with pro-tumorigenic activity [38]. Proinflammatory cytokines, particularly IL-6, secreted by both cancer cells and CAFs, determine a malignant-driven tumor microenvironment that promote CCA progression [39]. Consistently, CCA cells exhibit a very aggressive behavior, both in vitro and in vivo, when exposed to IL-6 [40-45]. IL-6 may contribute to CCA malignancy through a variety of pathways, including the inhibition of autophagy in CCA cells [30,41]. Autophagy (precisely, macroautophagy) is a quality-control process that directs redundant, aged, and non-functional cell components to lysosomes for their complete degradation [46]. Autophagy runs regularly at basal level to maintain cell homeostasis and can be upregulated in response to endogenous or exogenous stresses [47]. Insufficient autophagy has been shown to favor carcinogenesis, and on the other hand its up-regulation may confer a survival advantage to cancer cells exposed to genotoxic, proteotoxic and metabolic stresses [48]. Thus, to exploit autophagy as a therapeutic target in cancer management it is fundamental to determine how it is dysregulated within the tumor [49].

It is now accepted the idea that the actual level and functional outcome of autophagy in cancer cells depend on several factors [50], precisely: 1. the genetic background (i.e., the mutation in relevant oncogenes and tumor suppressor genes as well as in genes controlling the regulatory pathways) of the cancer cell; 2. The presence of growth factors, and the level of inflammation and of secreted inflammatory factors in the TME; 3 . The structural and metabolic status of the TME (vascularization, availability of nutrients and of oxygen); 4 . The presence of epigenetic modifiers in the TME. Further, autophagy is a dynamic process that adjust its regulation in the cells depending on the microenvironment. Therefore, the expression of autophagy markers reflects the situation in the tumor mass context at the time of the analysis.

The literature on the involvement of autophagy in CCA development and progression reports some contradictory data about its pro-tumorigenic or anti-tumorigenic role [49]. For instance, in vitro experiments with CCA cells showed that disruption of BECLIN 1-dependent autophagy could either sensitize to or abrogate cytotoxicity by chemotherapeutic drugs [26,30]. On the other hand, data from CCA xenograft experiments in nude mice might not truly resemble the situation in humans because of the lack of an efficacious immune-inflammatory response. Indeed, the uncertainty about the role of autophagy in cholangiocarcinogesis and the therapeutic response and clinical outcome likely reflects our lack of knowledge about the functional relationship between the level of inflammation in the stroma and the actual level of autophagy in CCA cells. The present study attempts to fill in this gap of knowledge. Briefly, we found that: 1 . high IL-6 in CAFs-containing fibrotic area was significantly associated with a 
shorter 5-year overall survival; 2. the combined pattern of high LC3 and low p62 in CCA cells showed a significant correlation with the best overall survival; 3 . the pattern of $L / H / L$, representing a low inflammatory stroma (low IL-6 staining) and an efficient autophagy flux in cancer cells (high LC3 and low p62) was significantly associated with the best prognostic clinical outcome; 4 . The tumors with low stromal IL- 6 and high cancer cell LC3 expression showed more responsive to chemotherapy than tumors with any other combination. To generalize the conclusion of our study, we have interrogated the TCGA database for CCA. Unfortunately, the limited number of cases and the lack of complete information on the expression of autophagy markers do not allow to draw conclusions statistically significant with regard to their prognostic value, though the available data consistently suggest that increased autophagy flux (with high LC3 and low p62) associates with better prognosis. In apparent contradiction, the TCGA data also indicated that CCA with high expression of BECN1 (one of the most important autophagy gene) had worse prognosis. However, a more depth analysis revealed that many of these patients were not subjected to chemotherapy. In addition, many CCAs with high BECN1 mRNA were indeed expressing low LC3 mRNA, indicating that autophagy in those samples was not active. In fact, the functional link between BECLIN1-autophagy and clinical outcome should be based on the true assessment of autophagy at protein level with particular attention to the expression of BECLIN 1, LC3 and p62 along with that of BCL-2 (an inhibitory interactor of BECLIN 1 that confers apoptosis resistance) in the same cells [51]. Supporting this interpretation, it was found that CCAs with low expression of BECLIN 1 were significantly associated with lymph node metastasis and worse OS, while cases with no lymph node invasion showed the highest expression of BECLIN 1 [24, 32]. Higher levels of LC3, BECLIN 1 and p62/SQSTM1 proteins were found in specimens of CCAs at any step of progression, from pre-invasive to invasive carcinomas, compared to non-neoplastic large bile ducts and peribiliary gland tissues [25]. The concomitant high expression of both LC3 and p62 could be interpreted as a defective autophagy flux since the very early steps of cholangiocarcinogenesis, which would be consistent with a tumor suppressive role of autophagy in cholangiocarcinogenesis. Thus, again, it appears fundamental to clearly assess the efficiency of the autophagy flux before drawing conclusion about the functional role of this process in cancer progression and chemotherapy response. To better understand this aspect, we attempted to reproduce (at best) in vitro the effect of IL- 6 on the chemotherapeutic response in human CCA cells and to assess the role of autophagy in such response. Primary CAFs were isolated from human CCA and genetically impaired to produce IL- 6 through specific siRNA transfection. CCA cells were then exposed to 5-FU (a prototype chemotherapeutic for CCA treatment) diluted in the conditioned medium of control (scramble siRNA-transfected) or of IL- 6 depleted (IL-6 siRNA-transfected) CAFs, and cell growth, colony formation and apoptosis were assessed. IL- 6 rich medium from CAFs stimulated CCA growth and prevented 5-FU cytotoxicity along with inhibition of autophagy. Interestingly, 5-FU was more effective when administered in CCA cultures devoid of IL-6, and BAX-mediated apoptosis was shown to occur in CCA cells accumulating LC3-positive vacuoles. This outcome can be explained by the two-hit model [52], which predicts that in cells with active autophagy (first stressing hit) any additional stress (in this case, the chemotherapeutic) further stimulates autophagy precipitating autophagy-associated apoptosis. 
Our study has several clinical implications. First, it confirms the need for determining the level of stromal inflammation (namely, the level of CAFs and of IL-6) to better design the therapy and predicts the prognosis. Second, in view of a personalized treatment, assessing thoroughly autophagy with several markers is mandatory for a better stratification of the patients that could benefit of drugs specifically targeting autophagy. Third, it supports the inclusion of autophagy-enhancing drugs to improve the clinical response. In this regard, some natural products such as pristimerin [53], dihydroartemisinin [30], pterostilbene [54] and resveratrol [41] seem very promising. We propose to use such autophagy enhancing drugs along with navitoclax that has been shown to kill CAFs in CCA [23], or with resveratrol, that has been shown to switch-off the synthesis of IL-6 by CCA-derived CAFs [41].

\section{Conclusions}

This is the first demonstration that the correlation between IL- 6 in the stroma and of autophagy markers in cancer cells may provide a prognostic value and help to stratify responding and non-responding patients bearing the CCA. This study collectively suggested that the therapeutic strategy of autophagyenhancing drugs given along with an adjuvant chemotherapy is an approach to fighting the stromal inflammation to improve the better outcome of CCA patients.

\section{Abbreviations}

CAFs, cancer-associated fibroblast; CCA, cholangiocarcinoma; CoM, control medium; $\mathrm{Cl}$, confidence interval; HR, Hazard ratio; IL-6, interleukin-6; TCGA, the cancer genome altas; TMA, tumor microarray

\section{Declarations}

\section{Acknowledgments}

Not applicable

\section{Authors' contributions}

$\mathrm{NN}, \mathrm{Cl}$ and WL conceived and designed the study, which were performed the experiments by ST. ST, CV, AF, NK, PS and AT collected and interpreted the data. ST, NN and Cl analyzed the data and wrote the manuscript. All authors reviewed the results and approved the final version of the manuscript.

\section{Funding}

This work was supported by Khon Kaen University to NN, a grant from a Mid-Career Grant (RSA5980012) of Thailand Research Fund to NN, a grant from Khon Kaen University to NN (KKU63), and a grant from CASCAP Program to NN. AF is recipient of a post-doctoral fellowship "Paolina Troiano" (id. 24094) granted by Associazione Italiana per la Ricerca sul Cancro (AIRC, Milan, Italy). CV was supported with a fellowship from Associazione per la Ricerca Medica Ippocrate-Rhazi (ARM-IR, Novara, Italy). 
Availability of data and materials

The data sets used and analyzed during the current study are available from the corresponding author on reasonable request.

\section{Ethics approval and consent to participate}

This study was approved by The Human Research Ethics Committee, Khon Kaen University, approved the research protocol (\#HE601063), all patients in accordance with the Declaration of Helsinki and its later revision.

\section{Consent for publication}

All patients involved in this study agree the publication of the data.

\section{Competing interests}

The authors have declared that no conflict of interest exists.

\section{References}

1. Blechacz B, Gores GJ. Cholangiocarcinoma: advances in pathogenesis, diagnosis, and treatment. Hepatology. 2008;48:308-21.

2. Doherty B, Nambudiri VE, Palmer WC. Update on the diagnosis and treatment of cholangiocarcinoma. Curr Gastroenterol Rep. 2017;19:2.

3. Zhang XF, Dong M, Pan YH, Chen JN, Huang XQ, Jin Y, et al. Expression pattern of cancer-associated fibroblast and its clinical relevance in intrahepatic cholangiocarcinoma. Hum Pathol. 2017;65:92100.

4. Prueksapanich P. Piyachaturawat P, Aumpansub P, Ridtitid W, Chaiteerakij R, Rerknimitr R. Liver flukeassociated biliary tract cancer. Gut Liver. 2018;12(3):236-45.

5. Franken LC, Schreuder AM, Roos E, Dieren SV, Busch OR, et al. Morbidity and mortality after major liver resection in patients with perihilar cholangiocarcinoma: A systematic review and meta-analysis. Surgery. 2019;165(5):918-28.

6. Moris D, Kostakis ID, Machairas N, Prodromidou A, Tsilimigras DI, Ravindra KV, et al. Comparison between liver transplantation and resection for hilar cholangiocarcinoma: A systematic review and meta-analysis. PloS One. 2019;14(7):e0220527.

7. Kennedy L, Hargrove L, Demieville J, Francis N, Seils R, Villamaria S, et al. Recent advances in understanding cholangiocarcinoma. F1000Res. 2017;6:1818.

8. Valle J, Wasan H, Palmer DH, Cunningham D, Anthoney A, Maraveyas A, et al. Cisplatin plus gemcitabine versus gemcitabine for biliary tract cancer. N Eng J Med. 2010;362(14):1273-81. 
9. Luvira V, Eurboonyanun Ch, Bhudhisawasdi V, Pugkhem A, Pairojkul Ch, Luvira V, et al. Patterns of recurrence after resection of mass-forming type intrahepatic Cholangiocarcinomas. Asian Pac $\mathrm{J}$ Cancer Prev. 2017;17(10):4735-9.

10. Watanabe A, Suzuki H, Yokobori T, Tsukagoshi M, Altan B, Kubo N, et al. Stathmin1 regulates p27 expression, proliferation and drug resistance, resulting in poor clinical prognosis in cholangiocarcinoma. Cancer Sci. 2014;105(6):690-6.

11. Wirasorn K, Ngamprasertchai T, Khuntikeo N, Pakkhem A, Ungarereevittaya P, Chindaprasirt J, et al. Adjuvant chemotherapy in resectable cholangiocarcinoma patients. J Gastroenterol Hepatol. 2013;28(12):1885-91.

12. Wardell CP, Fujita M, Yamada T, Simbolo M, Fassan M, Karlic R, et al. Genomic characterization of biliary tract cancers identifies driver genes and predisposing mutations. J Hepatol. 2018;68(5):95969.

13. Zhong W, Dai L, Liu J, Zhou S. Cholangiocarcinoma-associated genes identified by integrative analysis of gene expression data. Mol Med Rep. 2018;17(4):5744-53.

14. Zhang C, Zhang B, Meng D, Ge C. Comprehensive analysis of DNA methylation and gene expression profiles in cholangiocarcinoma. Cancer Cell Int. 2019;19:352.

15. Lowery MA, Ptashkin R, Jordan E, Berger MF, Zehir A, Capanu M, et al. Comprehensive molecular profiling of intrahepatic and extrahepatic cholangiocarcinomas: potential targets for intervention. Clin Cancer Res. 2018;24(17):4154-61.

16. Ueasilamongkol P, Khamphaya T, Guerra MT, Rodrigues MA, Gomes DA, Kong Y, et al. Type 3 inositol 1, 4, 5-trisphosphate receptor is increased and enhances malignant properties in cholangiocarcinoma. Hepatology. 2020;71(2):583-99.

17. Sittithumcharee G, Suppramote O, Vaeteewoottacharn K, Sirisuksakun C, Jamnongsong S, Laphanuwat $\mathrm{P}$, et al. Dependency of Cholangiocarcinoma on Cyclin D-Dependent Kinase Activity. Hepatology. 2019;70(5):1614-30.

18. Ishida K, Osakabe M, Eizuka M, Tai S, Sugimoto R, Fujita Y, et al. The expression of gastrointestinal differentiation markers in extrahepatic cholangiocarcinoma: clinicopathological significance based on tumor location. Hum Pathol. 2019;92:91-100.

19. Wang Y, Li J, Xia Y, Gong R, Wang K, Yan Z, et al. Prognostic nomogram for intrahepatic cholangiocarcinoma after partial hepatectomy. J Clin Oncol. 2013;31(9):1188-95.

20. Sasaki M, Matsubara T, Kakuda Y, Sato Y, Nakanuma Y. Immunostaining for polycomb group protein EZH2 and senescent marker p16INK4a may be useful to differentiate cholangiolocellular carcinoma from ductular reaction and bile duct adenoma. Am J Surg Pathol. 2014;38(3):364-9.

21. Sohal DP, Shrotriya S, Abazeed M, Cruise M, Khorana A. Molecular characteristics of biliary tract cancer. Crit Rev Oncol Hematol. 2016;107:111-8.

22. Simile MM, Bagella P, Vidili G, Spanu A, Manetti R, Seddaiu MA, et al. Targeted therapies in cholangiocarcinoma: emerging evidence from clinical trials. Medicina. 2019;55(2):42. 
23. Mertens JC, Fingas CD, Christensen JD, Smoot RL, Bronk SF, Werneburg NW, et al. Therapeutic effects of deleting cancer-associated fibroblasts in cholangiocarcinoma. Cancer Res. 20113;73(2):897-907.

24. Wang TT, Cao QH, Chen MY, Xia Q, Fan XJ, Ma XK, et al. Beclin 1 deficiency correlated with lymph node metastasis, predicts a distinct outcome in intrahepatic and extrahepatic cholangiocarcinoma. PLoS One. 2013;8(11):e80317.

25. Sasaki M, Nitta T, Sato Y, Nakanuma Y. Autophagy may occur at an early stage of cholangiocarcinogenesis via biliary intraepithelial neoplasia. Hum Pathol. 2015;46(2):202-9.

26. Hou YJ, Dong WL, Tan YX, Yang GZ, Pan YF, Li Z, et al. Inhibition of active autophagy induces apoptosis and increases chemosensitivity in cholangiocarcinoma. Lab Invest. 2011;91(8):1146-57.

27. Folkerts H, Hilgendorf S, Vellenga E, Bremer E, Wiersma VR. The multifaceted role of autophagy in cancer and the microenvironment. Med Res Rev. 2019;39(2):517-60.

28. Mowers EE, Sharifi MN, Macleod KF. Functions of autophagy in the tumor microenvironment and cancer metastasis. FEBS J. 2018;285(10):1751-66.

29. Thuwajit $C$, Ferraresi A, Titone R, Thuwajit $P$, Isidoro $C$. The metabolic cross-talk between epithelial cancer cells and stromal fibroblasts in ovarian cancer progression: autophagy plays a role. Med Res Rev. 2018;38(4):1235-54.

30. Thongchot S, Vidoni C, Ferraresi A, Loilome W, Yongvanit P, et al. Dihydroartemisinin induces apoptosis and autophagy-dependent cell death in cholangiocarcinoma through a DAPK1-BECLIN1 pathway. Mol Carcinog. 2018;57(12):1735-50.

31. Namwat N, Puetkasichonpasutha J, Loilome W, Yongvanit P, Techasen A, Puapairoj A, et al. Downregulation of reversion-inducing-cysteine-rich protein with Kazal motifs (RECK) is associated with enhanced expression of matrix metalloproteinases and cholangiocarcinoma metastases. $J$ Gastroenterol. 2011;46(5):664-75.

32. Dong LW, Hou YJ, Tan YX, Tang L, Pan YF, Wang M, et al. Prognostic significance of Beclin 1 in intrahepatic cholangiocellular carcinoma. Autophagy. 2011;7(10):1222-9.

33. Huang JL, Hezel AF. Autophagy in intra-hepatic cholangiocarcinoma. Autophagy. 2012;8(7):1148-9.

34. Nitta T, Sato Y, Ren XS, Harada K, Sasaki M, Hirano S, et al. Autophagy may promote carcinoma cell invasion and correlate with poor prognosis in cholangiocarcinoma. Int J Ckin Exp Pathol. 2014;7(8):4913-21.

35. Qu X, Yu J, Bhagat G, Furuya N, Hibshoosh H, Troxel A, et al. Promotion of tumorigenesis by heterozygous disruption of the beclin 1 autophagy gene. J Clin Invest. 2003;112(12):1809-20.

36. Chuaysri C, Thuwajit P, Paupairoj A, Chau-In S, Suthiphongchai T, Thuwajit C. Alpha-smooth muscle actin-positive fibroblasts promote biliary cell proliferation and correlate with poor survival in cholangiocarcinoma. Oncol Rep. 2009;21(4):957-69.

37. Vaquero J, Aoudjehane L, Fouassier L. Cancer-associated fibroblasts in cholangiocarcinoma. Curr Opin Gastroenterol. 2020;36(2):63-9. 
38. Utispan K, Thuwajit P, Abiko Y, Charngkaew K, Paupairoj A, Chau-in S, et al. Gene expression profiling of cholangiocarcinoma-derived fibroblast reveals alterations related to tumor progression and indicates periostin as a poor prognostic marker. Mol Cancer. 2010;9:13.

39. Johnson C, Han Y, Hughart N, McCarra J, Alpini G, Meng F. Interleukin-6 and its receptor, key players in hepatobiliary inflammation and cancer. Transl Gastrointest Cancer. 2012;1(1):58-70.

40. Goydos JS, Brumfield AM, Frezza E, Booth A, Lotze MT, Carty SE. Marked elevation of serum interleukin-6 in patients with cholangiocarcinoma: validation of utility as a clinical marker. 1998;227(3):398-404.

41. Thongchot S, Ferraresi A, Vidoni C, Loilome W, Yongvanit P, Namwat N, et al. Resveratrol interrupts the pro-invasive communication between cancer associated fibroblasts and cholangiocarcinoma cells. Cancer Lett. 2018;430:160-71.

42. Tangkijvanich $P$, Thong-ngam $D$, Theamboonlers A, Hanvivatvong O, Kullavanijaya, et al. Diagnostic role of serum interleukin 6 and CA $19-9$ in patients with cholangiocarcinoma.

Hepatogastroenterology. 2004;51:15-9.

43. Frampton G, Invernizzi P, Bernuzzi F, Pae HY, Quinn M, Horvat D, et al. Interleukin-6-driven progranulin expression increases cholangiocarcinoma growth by an Akt-dependent mechanism. Gut. 2012;61(2):268-77.

44. Park J, Tadlock L, Gores GJ, Patel T. Inhibition of interleukin 6-mediated mitogen-activated protein kinase activation attenuates growth of a cholangiocarcinoma cell line. Hepatology. 1999;30(5):1128-33.

45. Daya M, Loilome W, Techasen A, Thanee M, SaNgiamwibool P, Titapun A, et al. Progranulin modulates cholangiocarcinoma cell proliferation, apoptosis, and motility via the PI3K/pAkt pathway. Onco Targets Ther. 2018;11:395-408.

46. Yu L, Chen Y, Tooze SA. Autophagy pathway: cellular and molecular mechanisms. Autophagy. 2018;14(2):207-15.

47. Kroemer G, Mariño G, Levine B. Autophagy and the integrated stress response. Mol Cell. 2010;40(2):280-93.

48. Chavez-Dominguez R, Perez-Medina M, Lopez-Gonzalez JS, Galicia-Velasco M, Aguilar-Cazares D. The double-edge sword of autophagy in cancer: From tumor suppression to pro-tumor activity. Front Oncol. 2020;10:578418.

49. Pérez-Hernández M, Arias A, Martínez-García D, Pérez-Tomás R, Quesada R, et al. Targeting autophagy for cancer treatment and tumor chemosensitization. Cancers (Basal). 2019;11(10):1599.

50. Ferraresi A, Girone C, Esposito A, Vidoni C, Vallino L, Secomandi E, et al. How autophagy shapes the tumor microenvironment in ovarian cancer. Front Oncol Accepted. 2020. doi:10.3389/fonc.2020.599915.

51. Valente G, Morani F, Nicotra G, Fusco N, Peracchio C, Titone R, et al. Expression and clinical significance of the autophagy proteins BECLIN 1 and LC3 in ovarian cancer. Biomed Res Int. 2014;2014:462658. 
52. Castino R, Isidoro C, Murphy D. Autophagy-dependent cell survival and cell death in an autosomal dominant familial neurohypophyseal diabetes insipidus in vitro model. FASEB J. 2005;19(8):1024-6.

53. Sun JM, Xu HT, Zhao L, Zhang YB, Kang PC, Song ZF, et al. Induction of cell-cycle arrest and apoptosis in human cholangiocarcinoma cells by pristimerin. J Cell Biochem. 2019;120(7):12002-9.

54. Wang $\mathrm{D}$, Guo $\mathrm{H}$, Yang $\mathrm{H}$, et al. Pterostilbene, an active constituent of blueberries, suppresses proliferation potential of human cholangiocarcinoma via enhancing the autophagic flux. Front Pharmacol. 2019;10:1238.

\section{Figures}


A

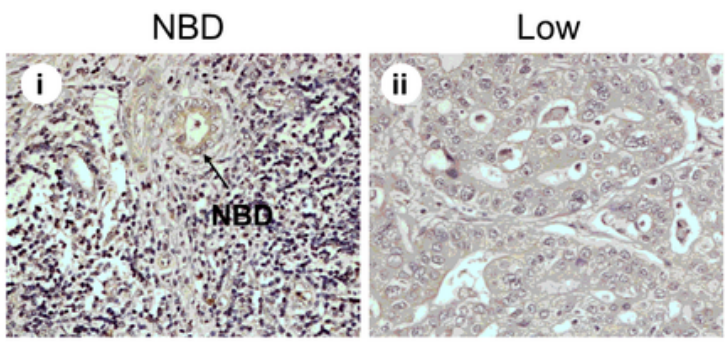

High

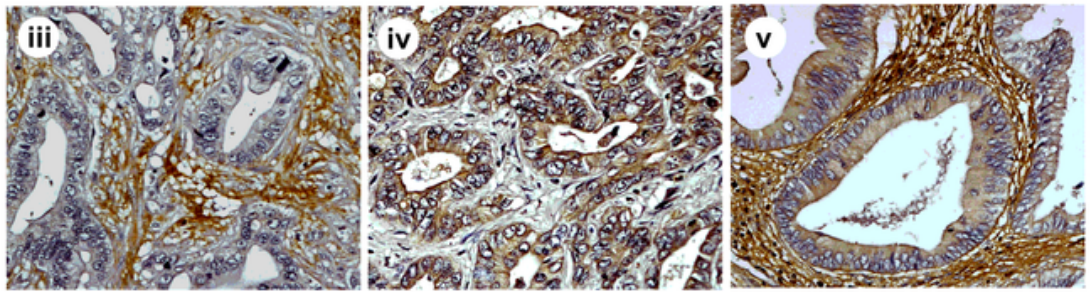

B

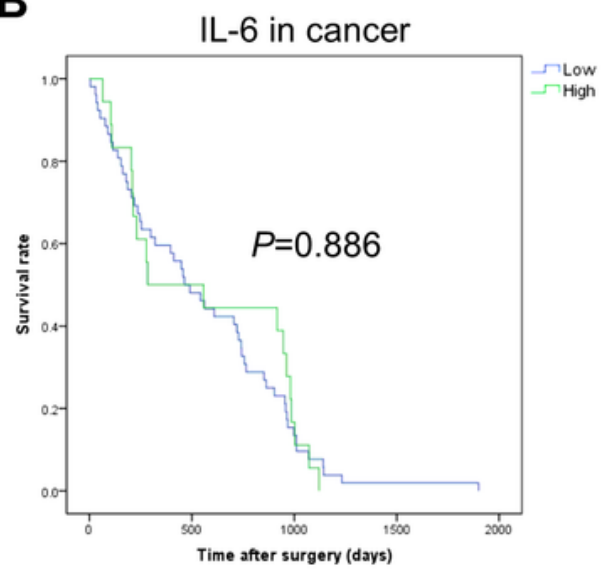

D

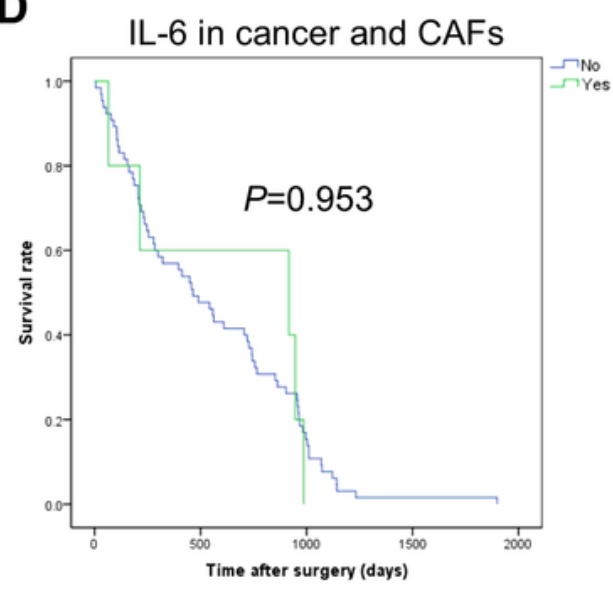

C

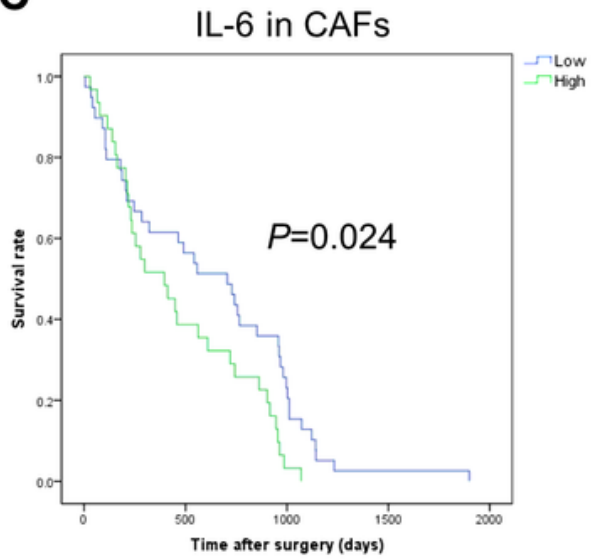

E

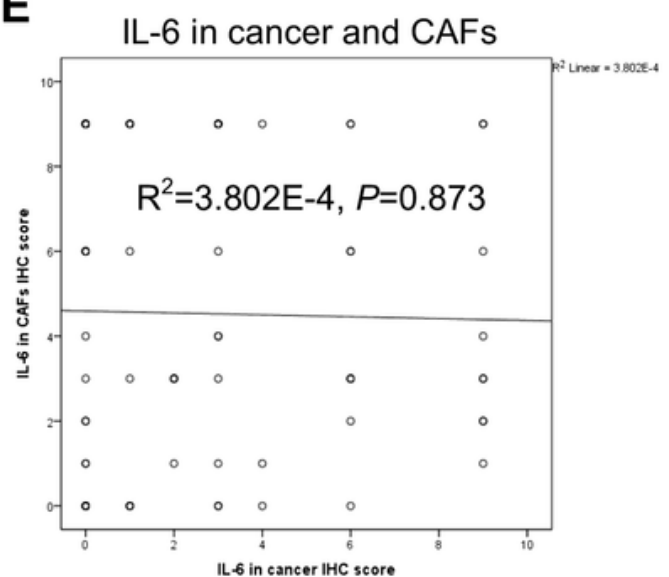

\section{Figure 1}

High expression of IL-6 in fibrotic stromal cells significantly correlates with shorter survival in CCA patients. a Representative images of IL-6 immunohistochemistry (IHC) in human CCA tissues showing faintly staining (negative) in normal bile duct (NBD) (i) and in some cancer areas (ii), while showing strongly intense (positive) in fibrotic area rich of CAFs (iii), in a few cancer areas (iv), and in both cancer epithelial cells and fibrotic areas (v). b-d Kaplan-Meier survival analysis for IL-6 IHC staining in cancer 
area (b), or in CAFs area (c), or in cancer and CAF area (d). e The relationship between IL-6 protein expression in cancer and CAFs is illustrated as scattered plots with linear regression lines. (Magnification 40X).

A
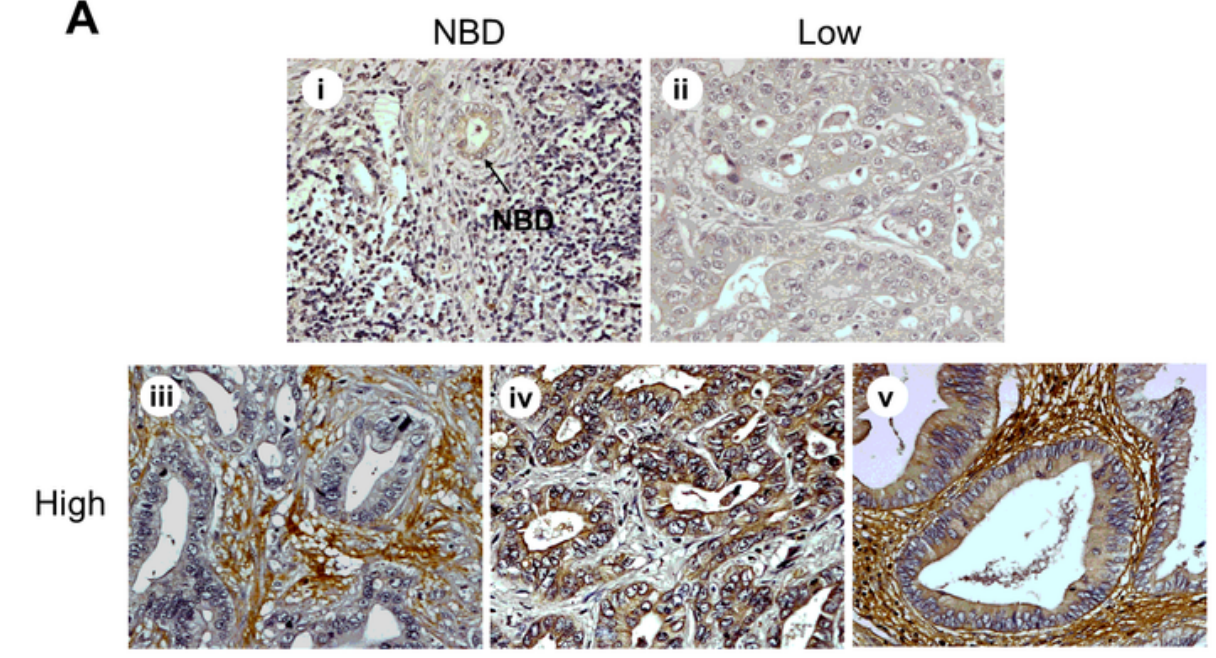

B

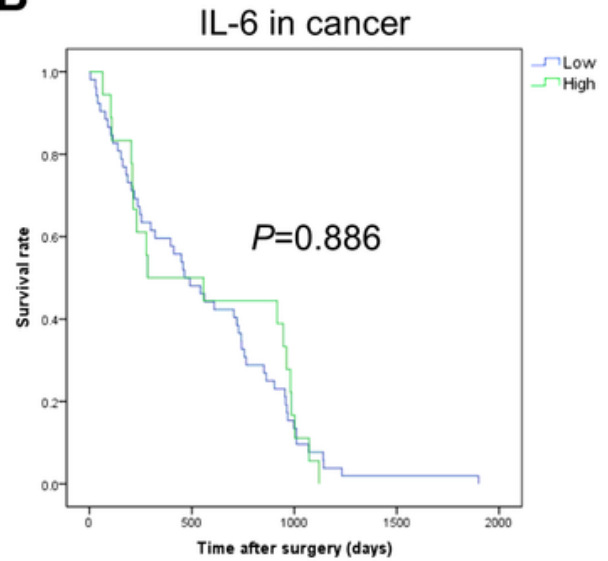

D

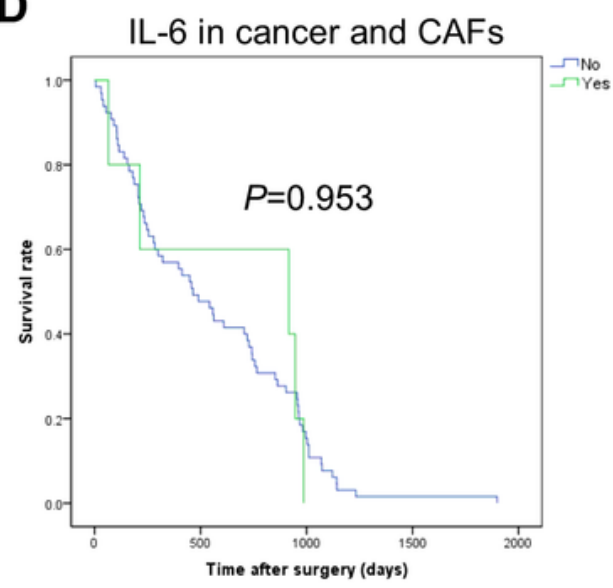

C

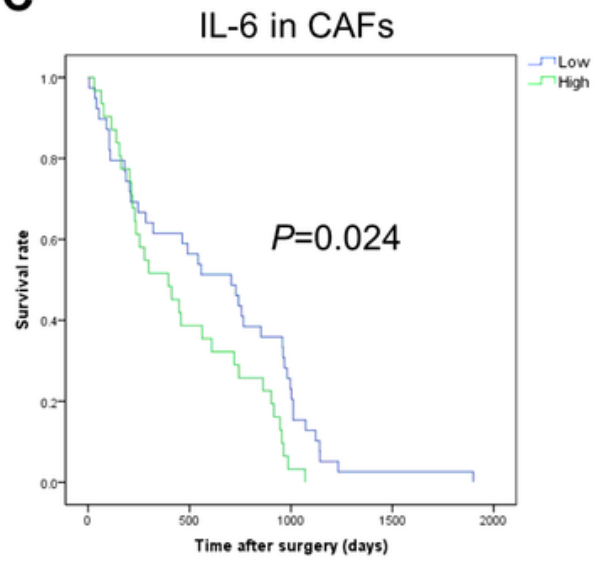

E

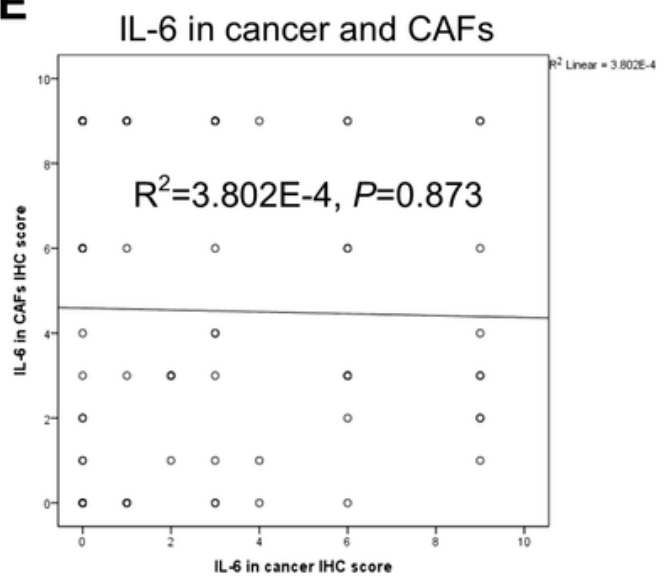

\section{Figure 1}

High expression of IL-6 in fibrotic stromal cells significantly correlates with shorter survival in CCA patients. a Representative images of IL-6 immunohistochemistry (IHC) in human CCA tissues showing 
faintly staining (negative) in normal bile duct (NBD) (i) and in some cancer areas (ii), while showing strongly intense (positive) in fibrotic area rich of CAFs (iii), in a few cancer areas (iv), and in both cancer epithelial cells and fibrotic areas (v). b-d Kaplan-Meier survival analysis for IL-6 IHC staining in cancer area (b), or in CAFs area (c), or in cancer and CAF area (d). e The relationship between IL-6 protein expression in cancer and CAFs is illustrated as scattered plots with linear regression lines. (Magnification 40X).

A

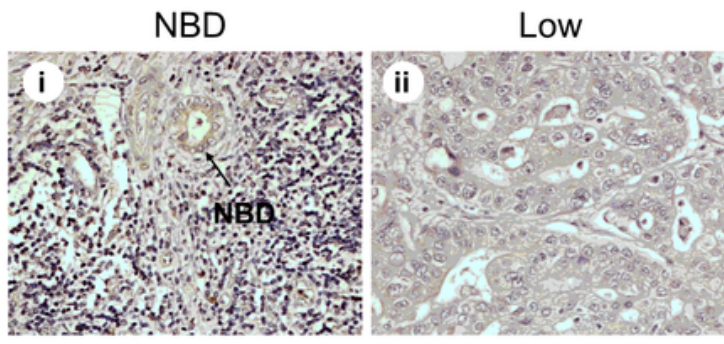

High

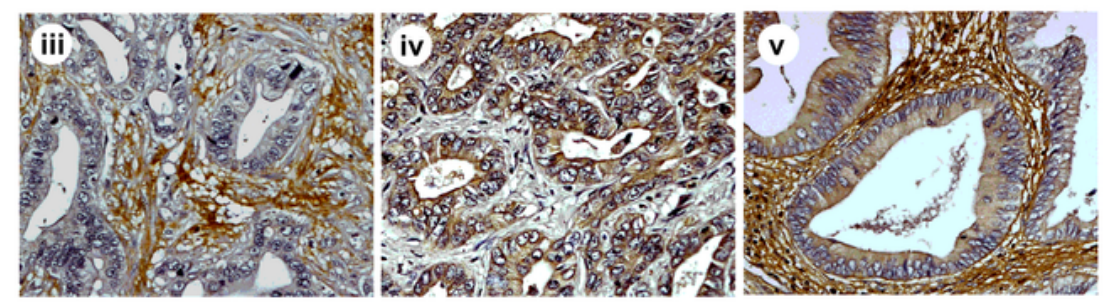

B

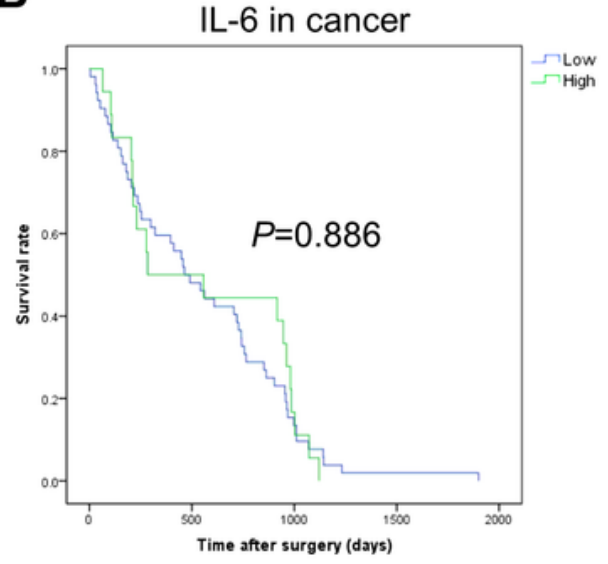

D

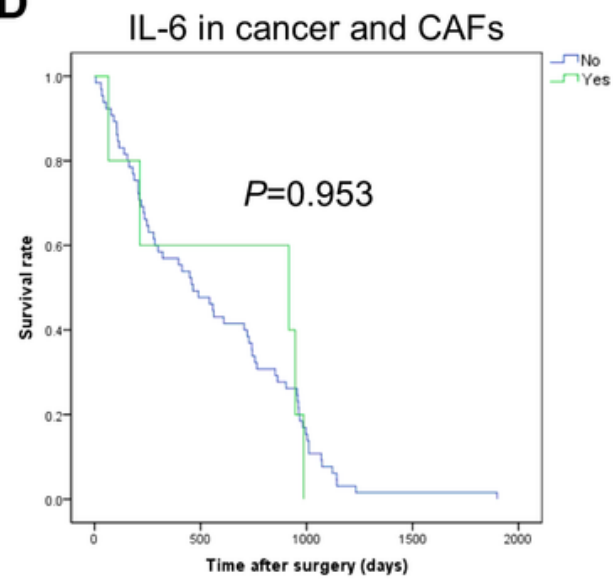

C

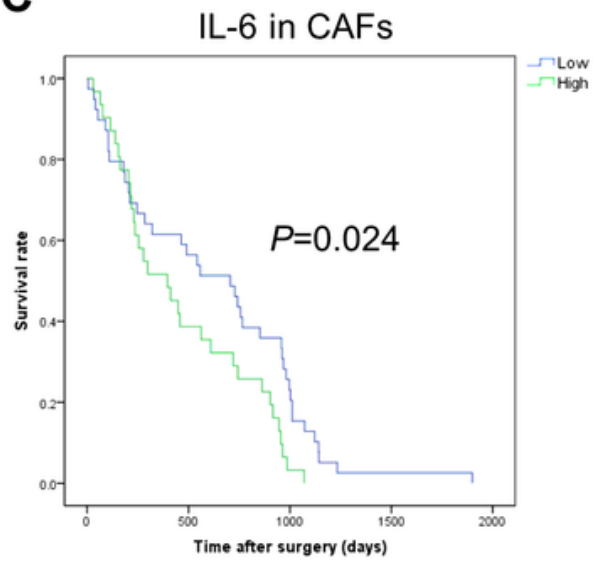

E

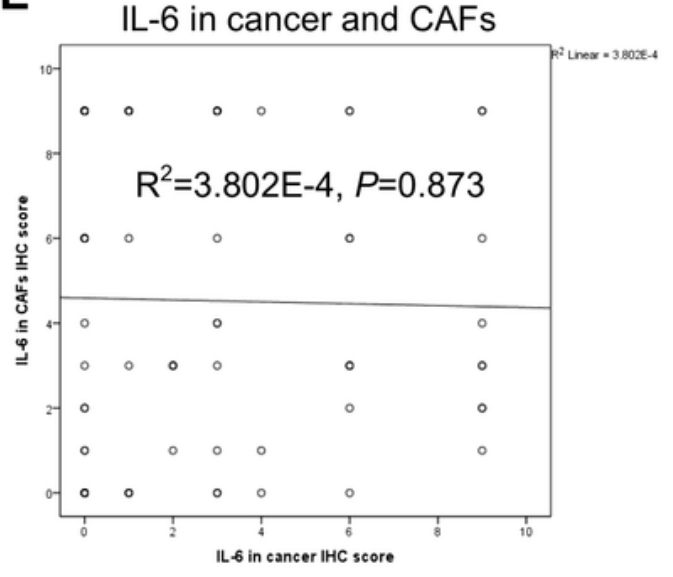


High expression of IL-6 in fibrotic stromal cells significantly correlates with shorter survival in CCA patients. a Representative images of IL-6 immunohistochemistry (IHC) in human CCA tissues showing faintly staining (negative) in normal bile duct (NBD) (i) and in some cancer areas (ii), while showing strongly intense (positive) in fibrotic area rich of CAFs (iii), in a few cancer areas (iv), and in both cancer epithelial cells and fibrotic areas (v). b-d Kaplan-Meier survival analysis for IL-6 IHC staining in cancer area (b), or in CAFs area (c), or in cancer and CAF area (d). e The relationship between IL-6 protein expression in cancer and CAFs is illustrated as scattered plots with linear regression lines. (Magnification 40X). 


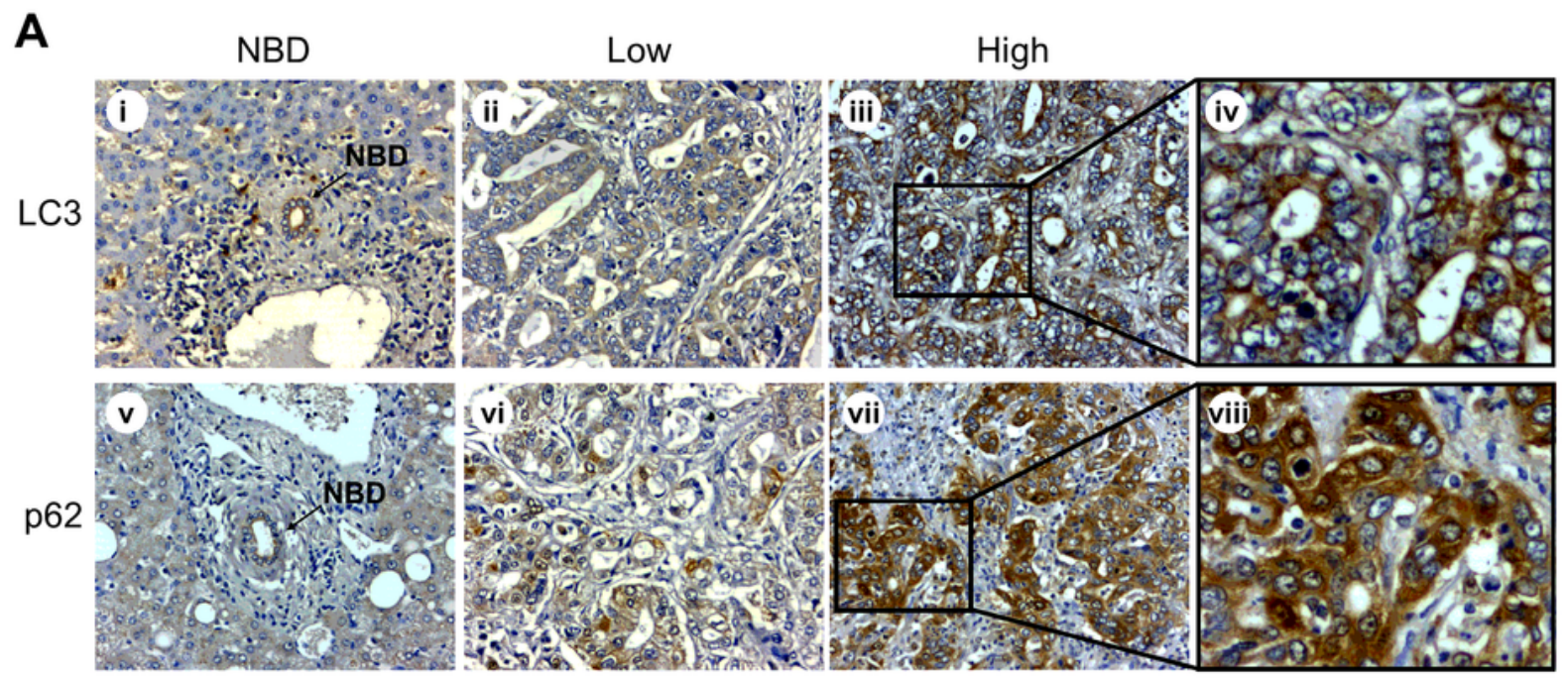

B

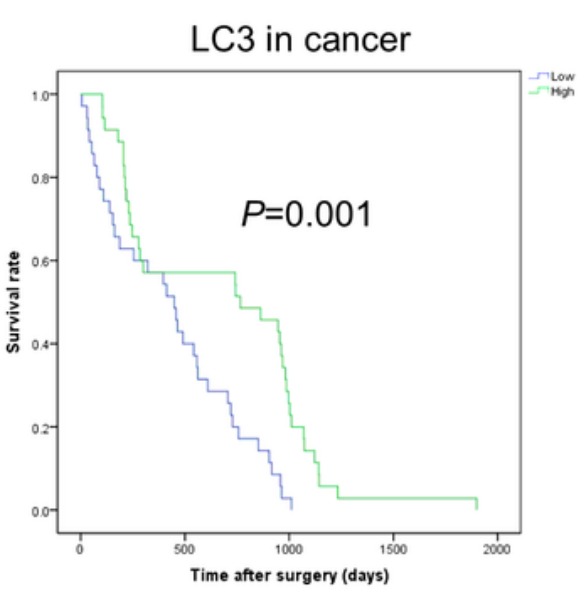

D

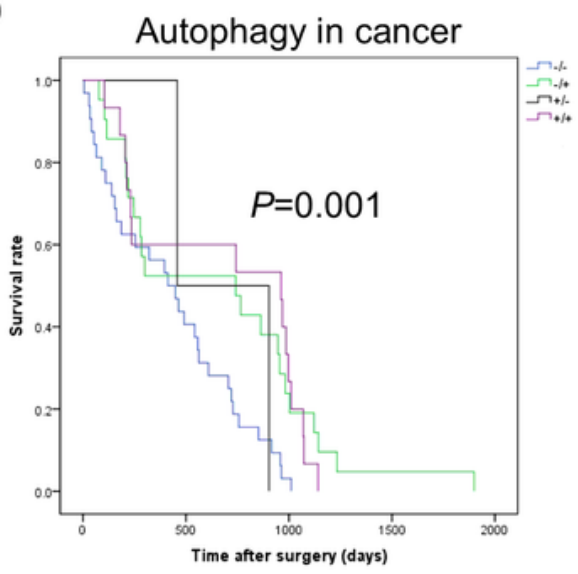

C

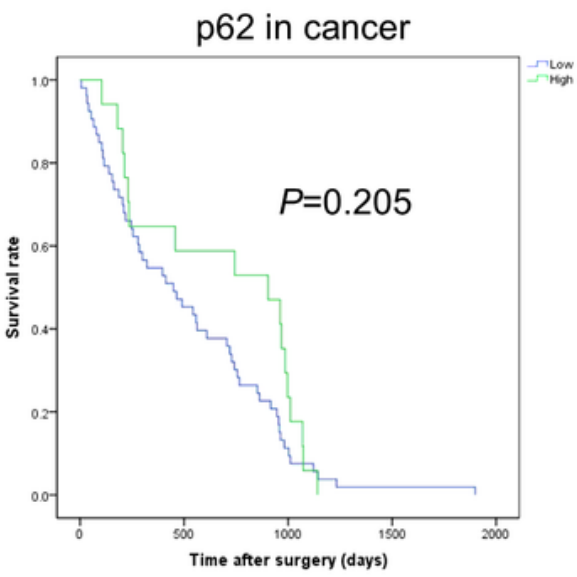

E

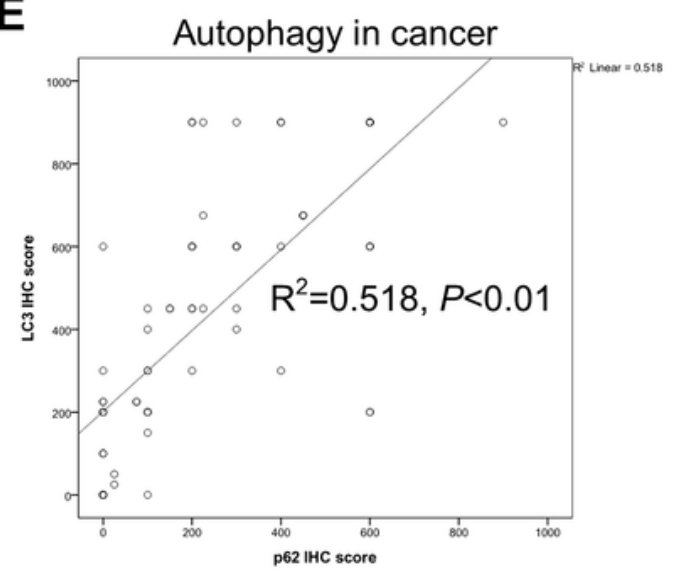

\section{Figure 2}

Immunohistochemistry expression of LC3 and p62 in cancer cells and its correlation with overall survival of CCA patients. a Representative cases showing LC3 and p62 immunostaining in normal bile ducts (NBD) (panels $i$ and v) and in cancer areas (panels ii-iv and vi-viii). Note that p62 staining is at all times higher than that of LC3 in the cancer area considered. Original magnification is $\times 200$. b-d Cumulative survival analysis shows a significant longer survival rate of CCA patients with high LC3 and low p62 
expression, indicative of ongoing autophagy. e The relationship between LC3B and cytoplasmic p62 expression exhibits positively correlation. (Magnification 20X and 40x).

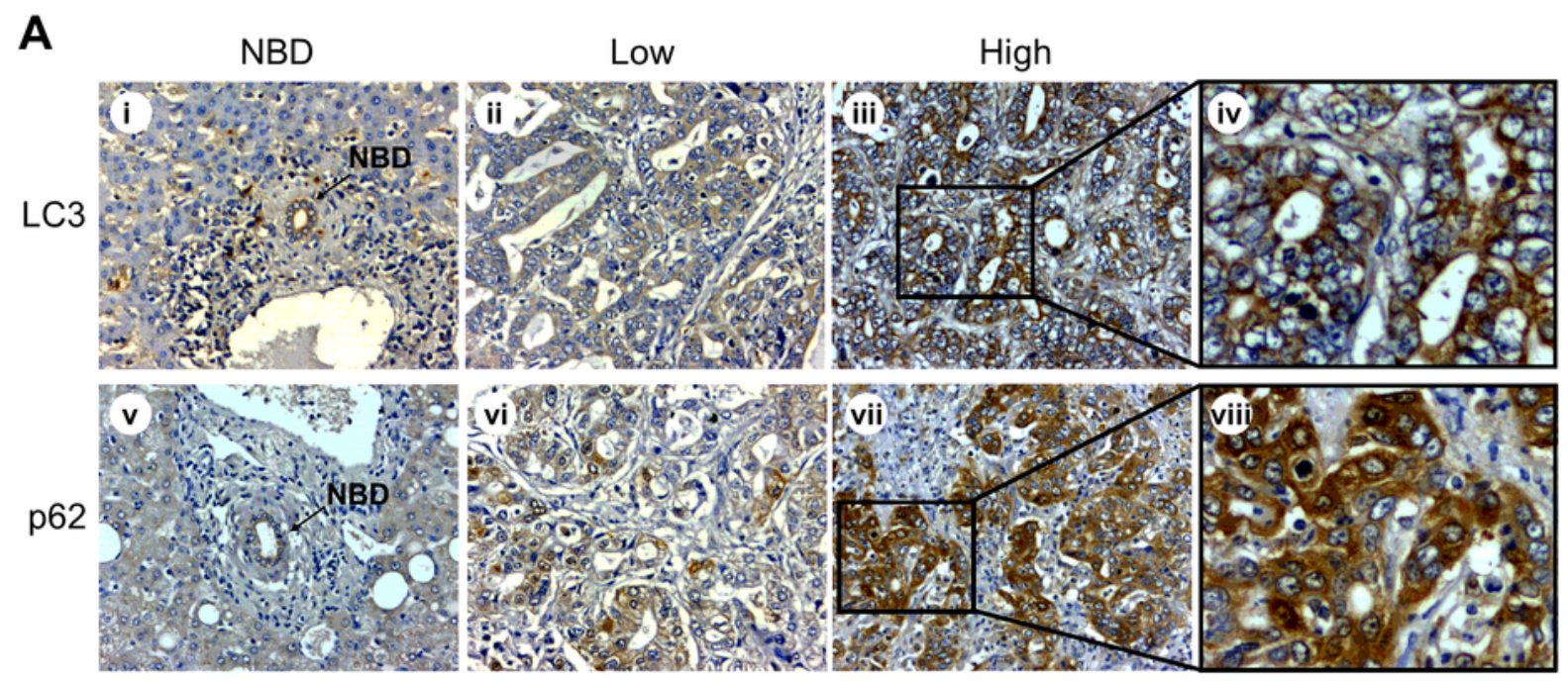

B

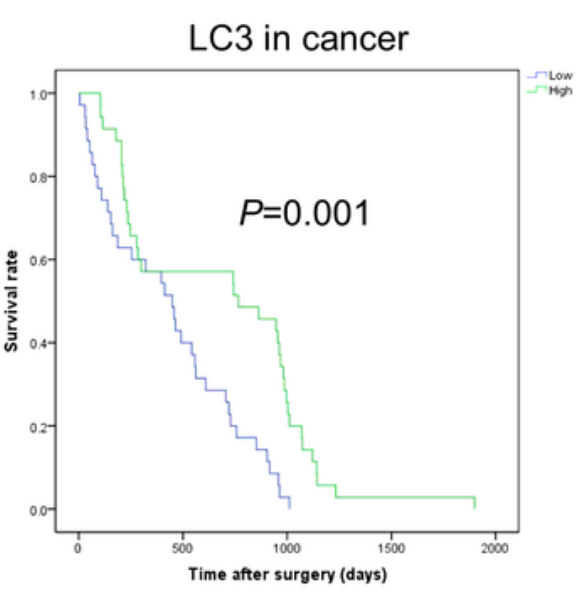

D

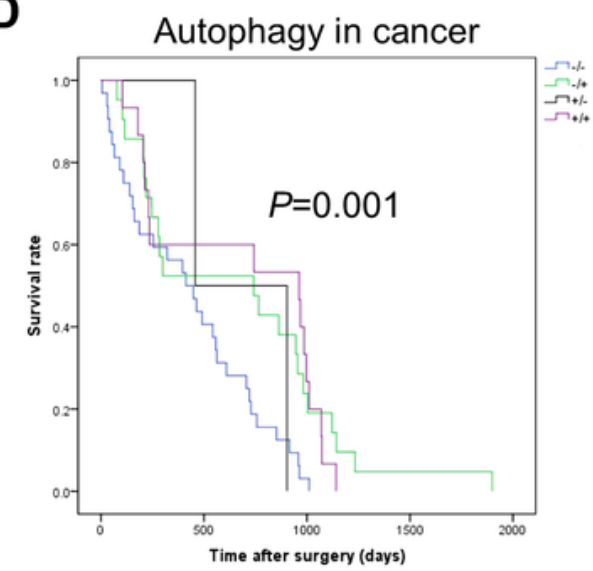

C

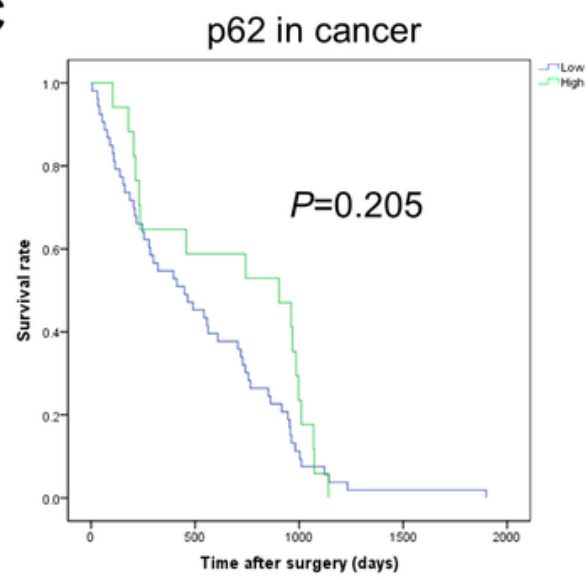

E

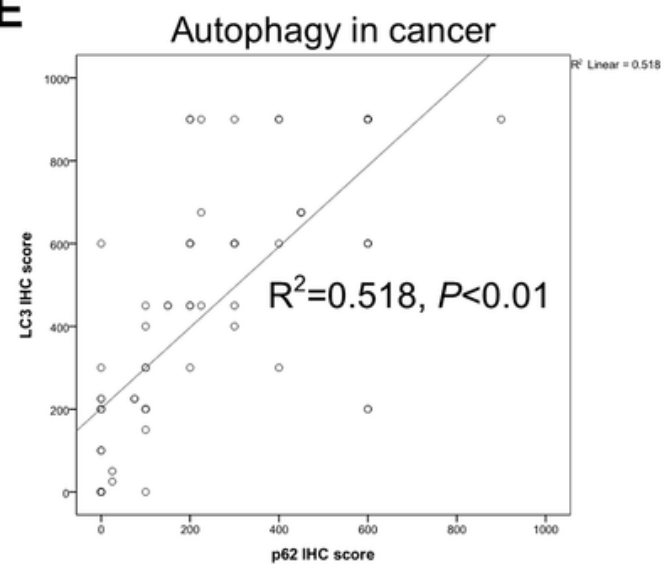

Figure 2

Immunohistochemistry expression of LC3 and p62 in cancer cells and its correlation with overall survival of CCA patients. a Representative cases showing LC3 and p62 immunostaining in normal bile ducts (NBD) (panels $i$ and v) and in cancer areas (panels ii-iv and vi-viii). Note that p62 staining is at all times 
higher than that of LC3 in the cancer area considered. Original magnification is $\times 200$. b-d Cumulative survival analysis shows a significant longer survival rate of CCA patients with high LC3 and low p62 expression, indicative of ongoing autophagy. e The relationship between LC3B and cytoplasmic p62 expression exhibits positively correlation. (Magnification 20X and 40x).

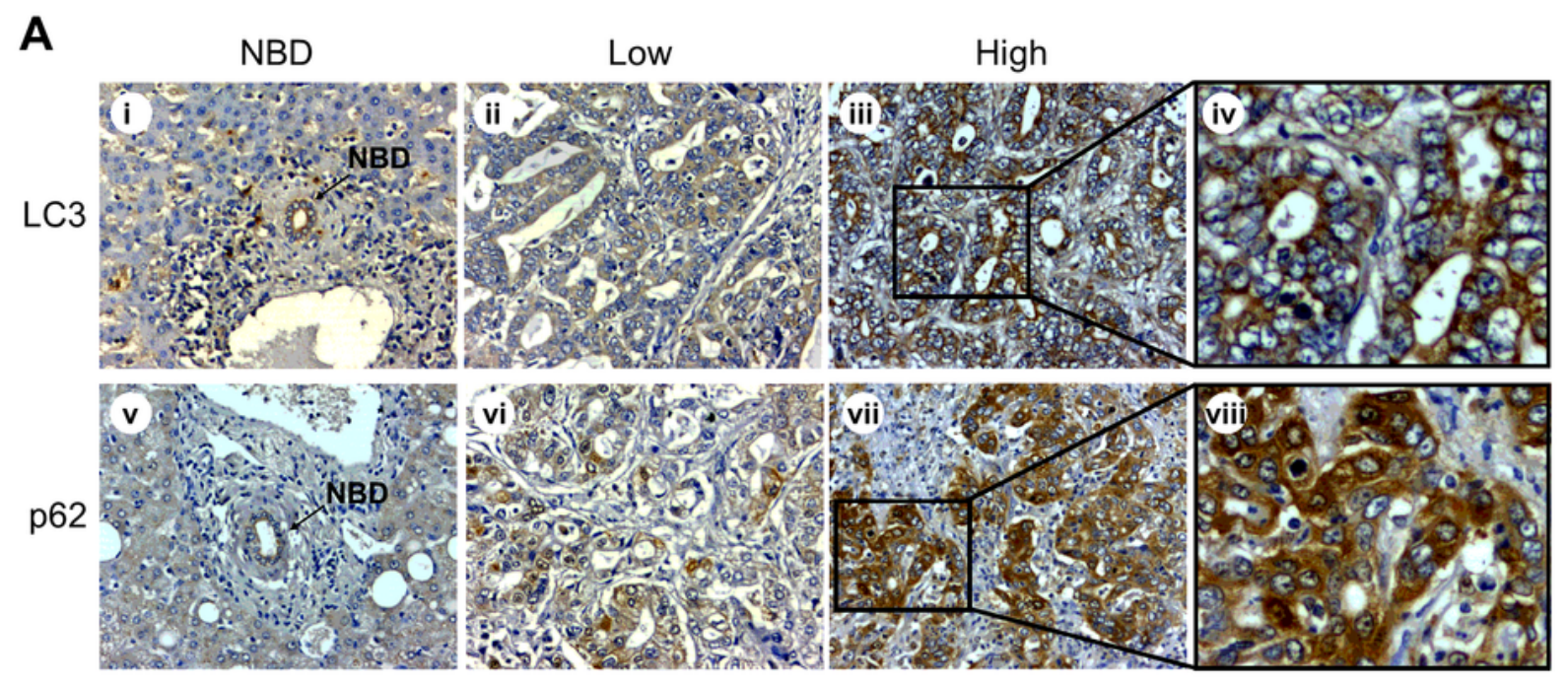

B

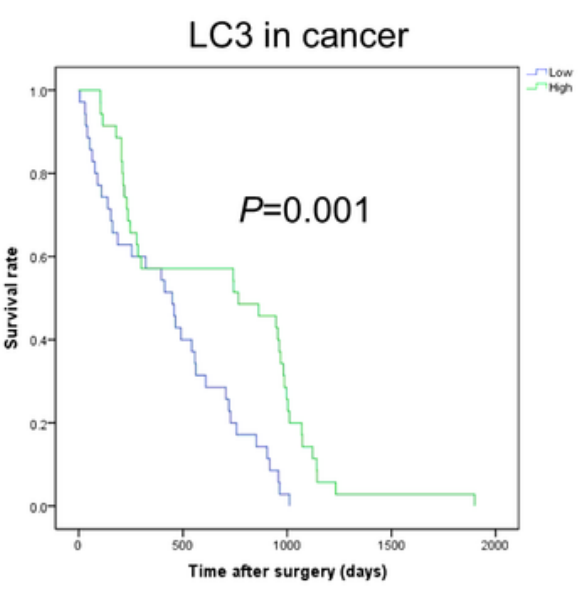

D

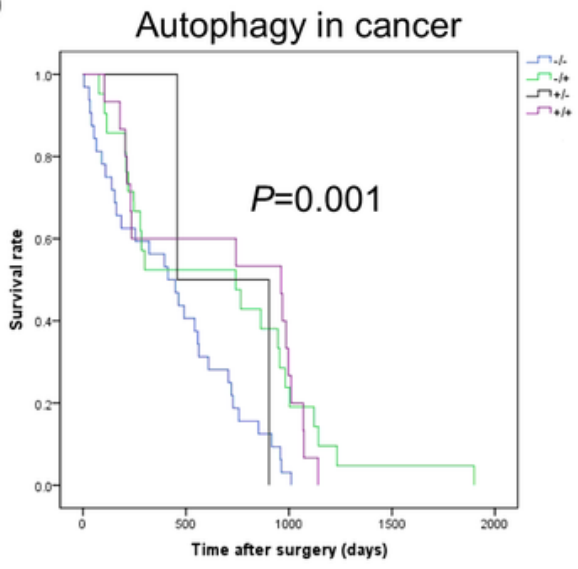

C

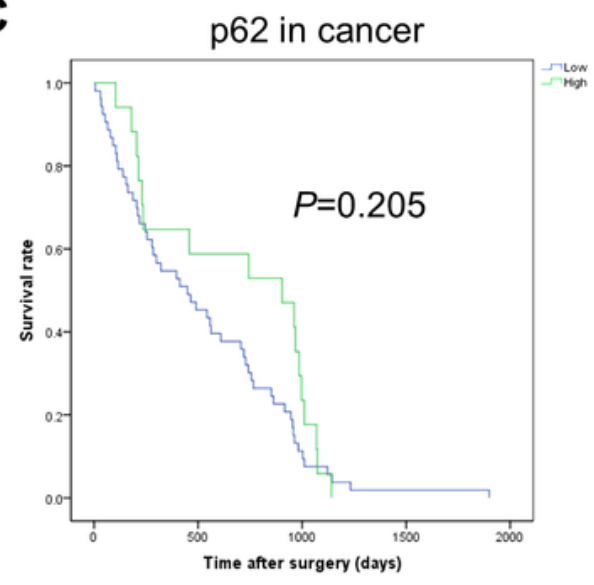

E

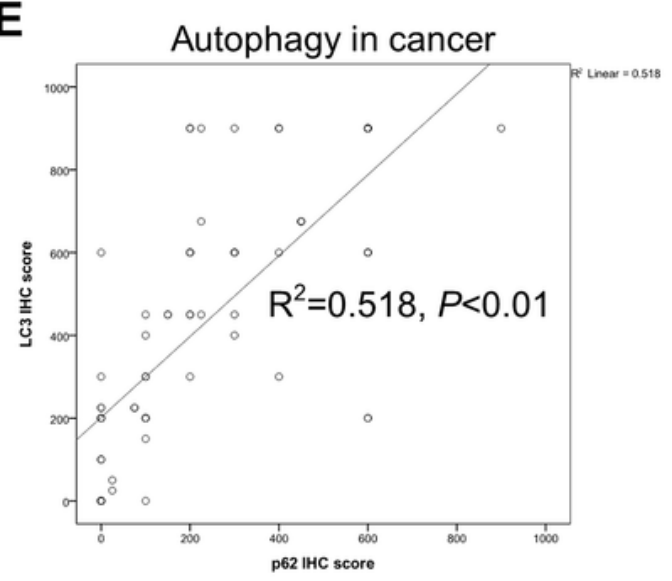

Figure 2 
Immunohistochemistry expression of LC3 and p62 in cancer cells and its correlation with overall survival of CCA patients. a Representative cases showing LC3 and p62 immunostaining in normal bile ducts (NBD) (panels $i$ and v) and in cancer areas (panels ii-iv and vi-viii). Note that $p 62$ staining is at all times higher than that of LC3 in the cancer area considered. Original magnification is $\times 200$. b-d Cumulative survival analysis shows a significant longer survival rate of CCA patients with high LC3 and low p62 expression, indicative of ongoing autophagy. e The relationship between LC3B and cytoplasmic p62 expression exhibits positively correlation. (Magnification 20X and 40x).

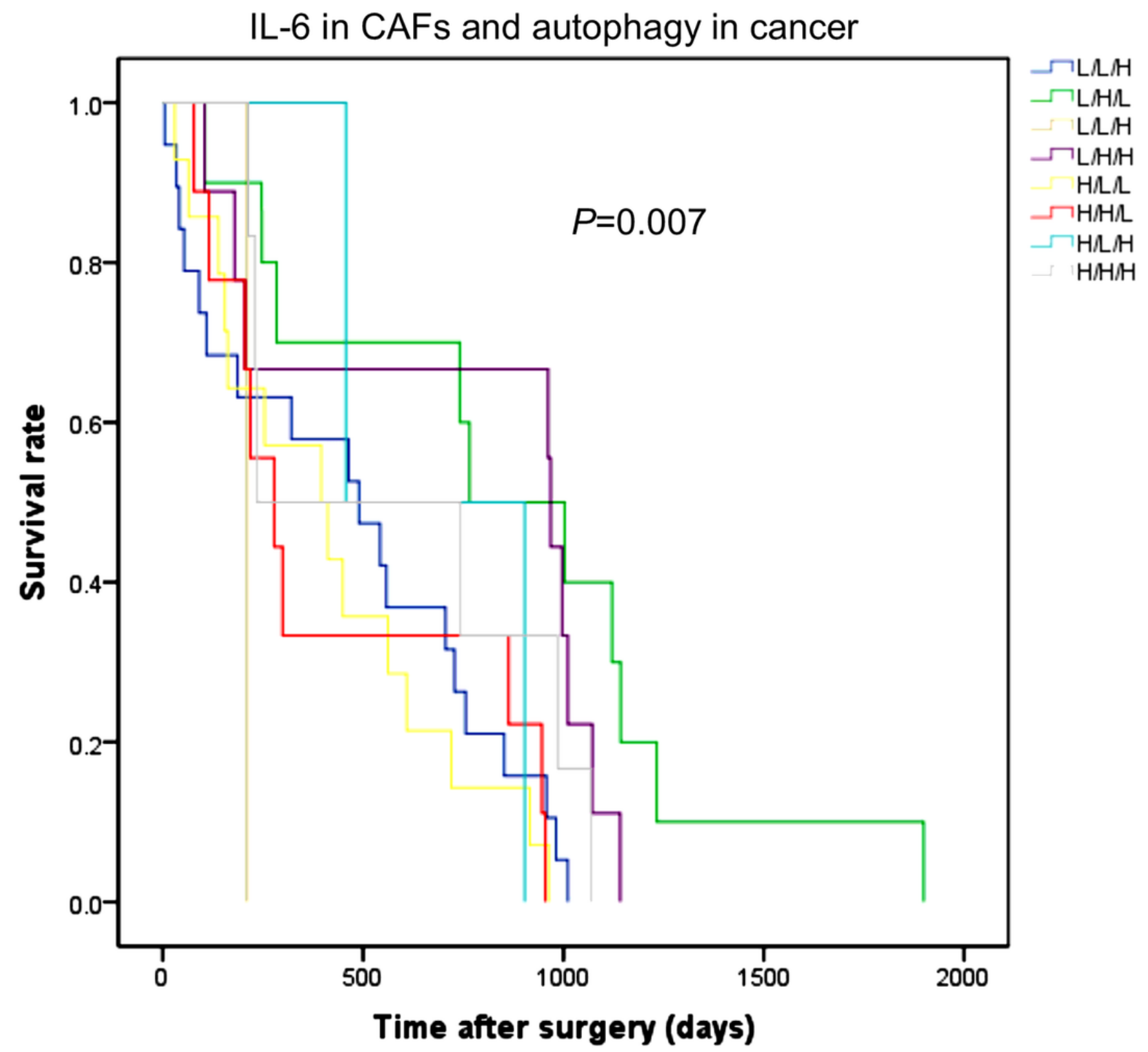

Figure 3 
The Kaplan-Meier curves of overall survival rates according to IL-6 in fibroblasts and LC3 and p62 expression in CCA epithelial cells. Combination of IHC expression of IL-6 in stromal fibrotic areas and of autophagy markers (LC3 and p62) in cancer cells were classified into 8 patterns as indicated in the text. Low IL-6, high LC3, and low p62 (L/H/L) expression significantly correlate with better overall survival rates (green line; $P=0.007$, log-rank test).

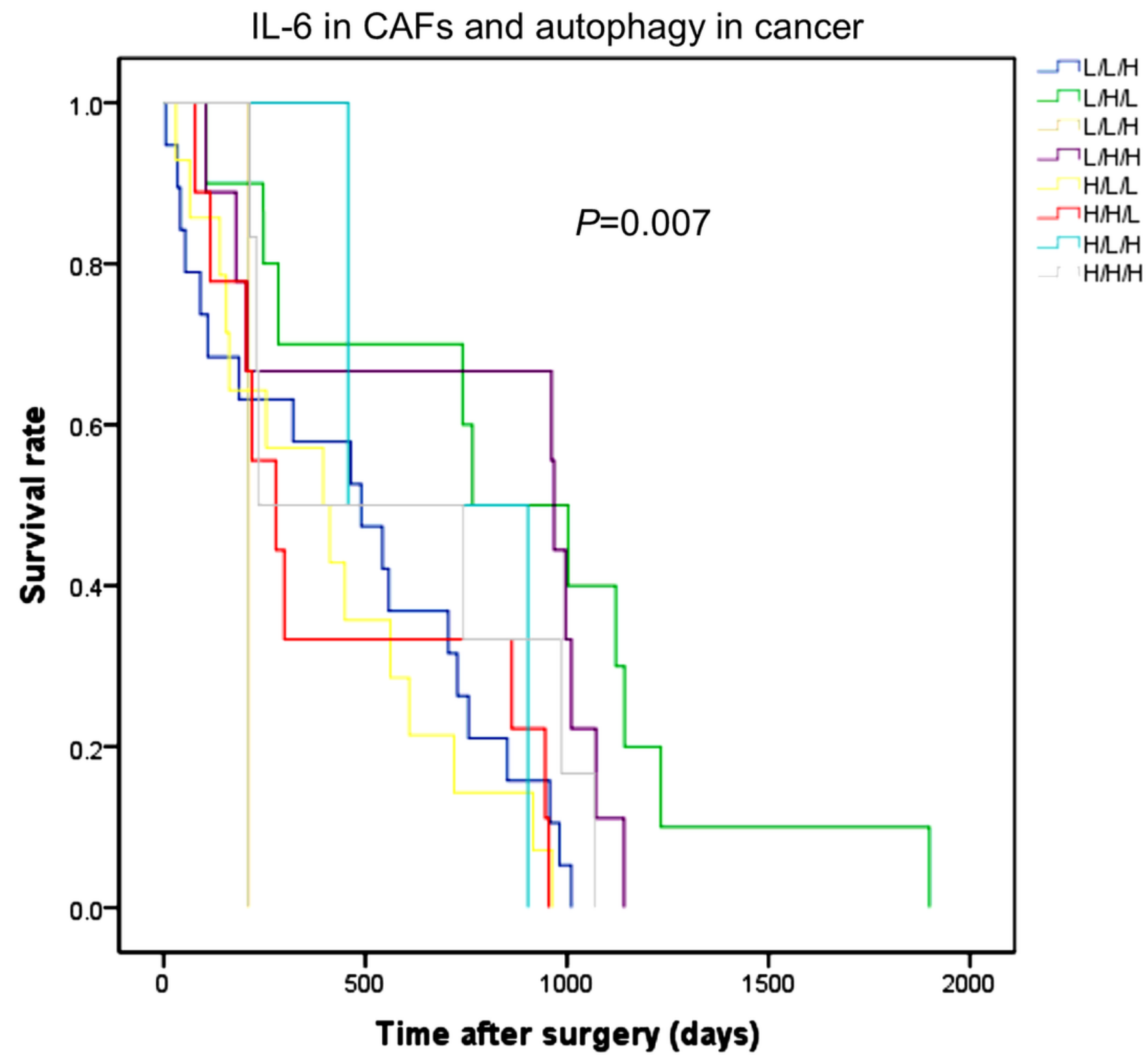

Figure 3

The Kaplan-Meier curves of overall survival rates according to IL- 6 in fibroblasts and LC3 and p62 expression in CCA epithelial cells. Combination of IHC expression of IL-6 in stromal fibrotic areas and of autophagy markers (LC3 and p62) in cancer cells were classified into 8 patterns as indicated in the text. 
Low IL-6, high LC3, and low p62 (L/H/L) expression significantly correlate with better overall survival rates (green line; $\mathrm{P}=0.007$, log-rank test).

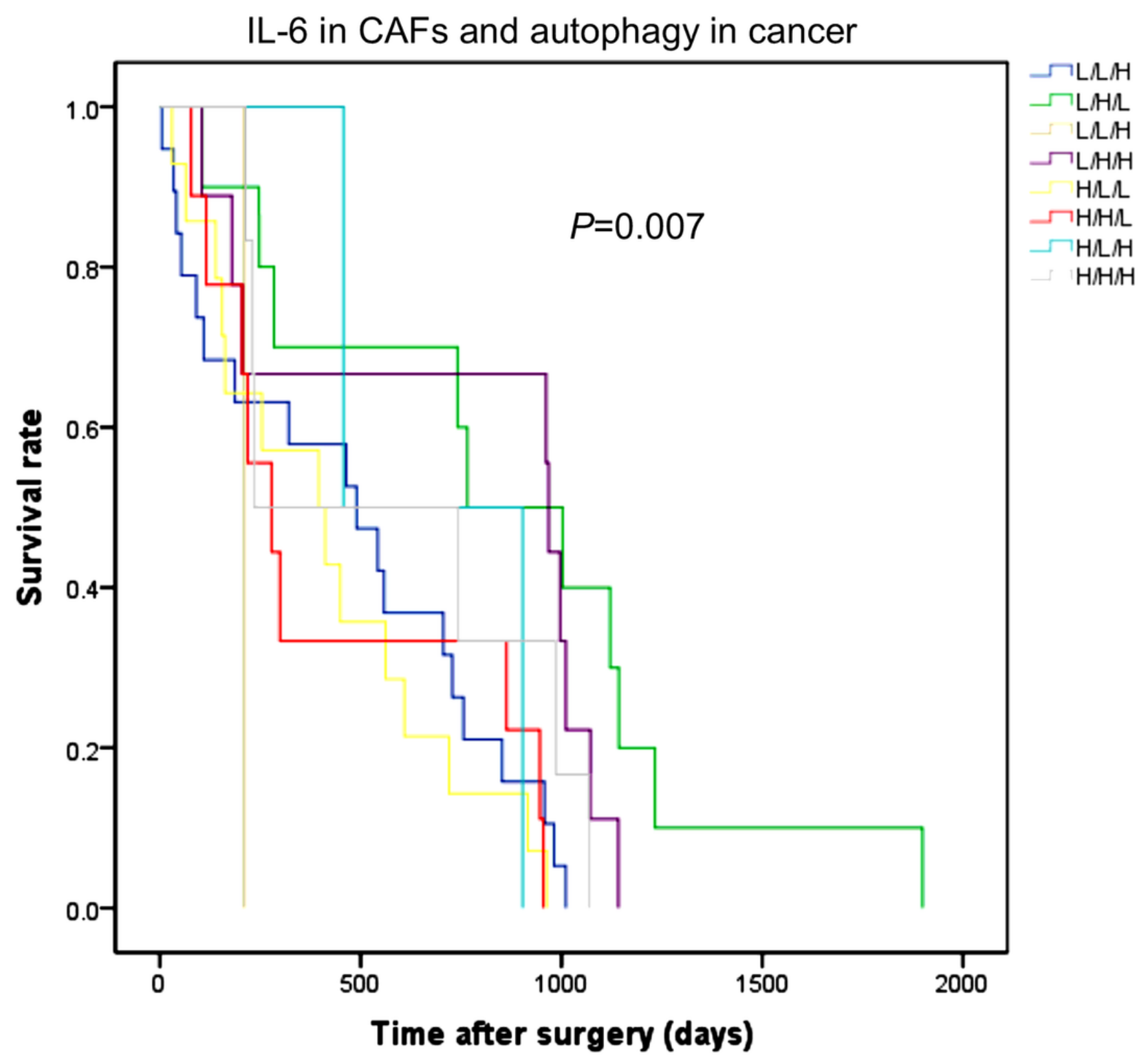

Figure 3

The Kaplan-Meier curves of overall survival rates according to IL-6 in fibroblasts and LC3 and p62 expression in CCA epithelial cells. Combination of IHC expression of IL-6 in stromal fibrotic areas and of autophagy markers (LC3 and p62) in cancer cells were classified into 8 patterns as indicated in the text. Low IL-6, high LC3, and low p62 (L/H/L) expression significantly correlate with better overall survival rates (green line; $P=0.007$, log-rank test). 


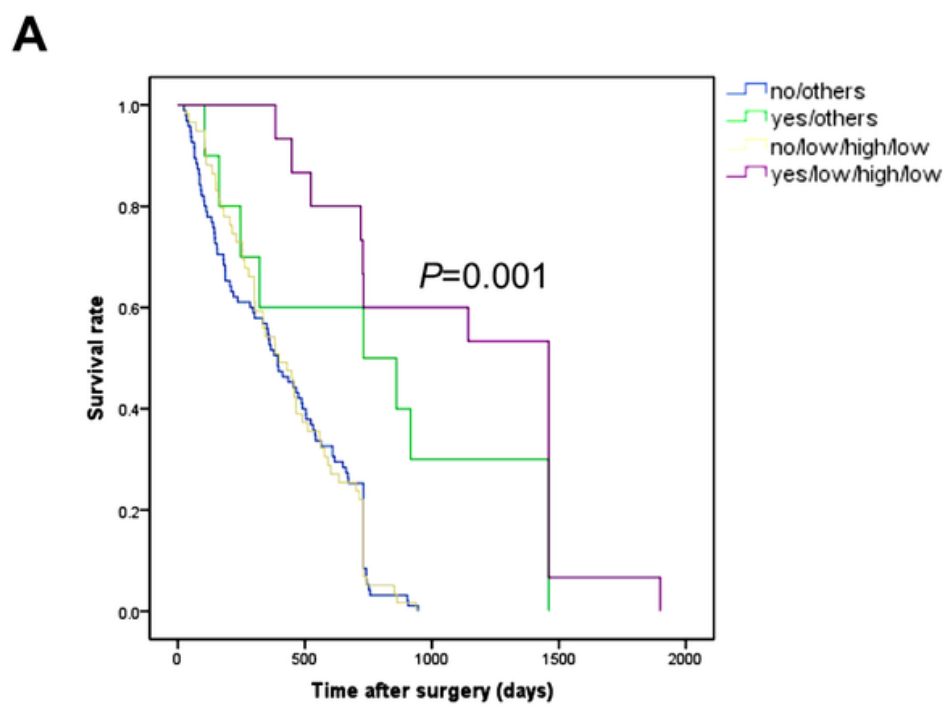

B

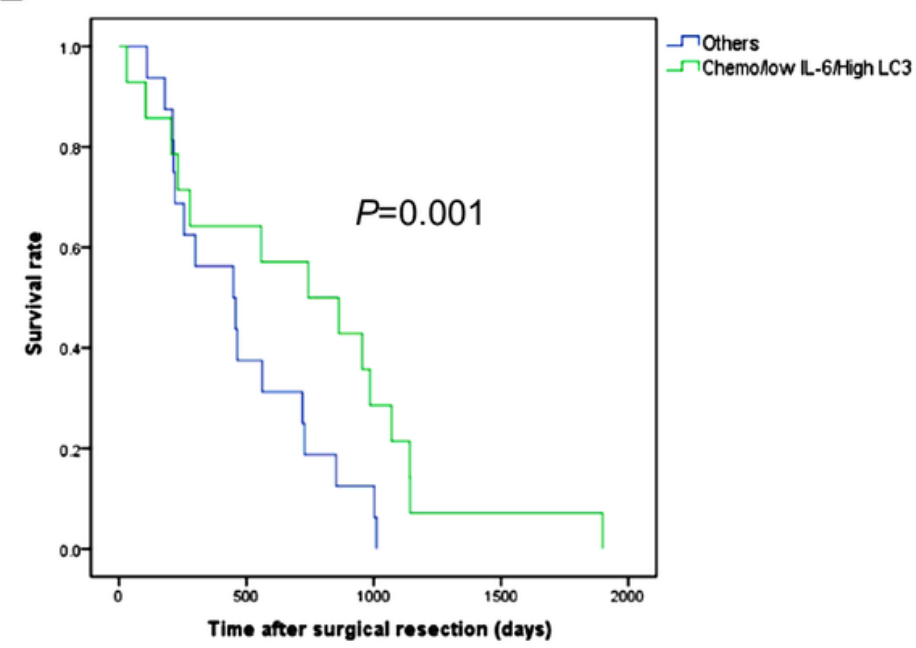

C

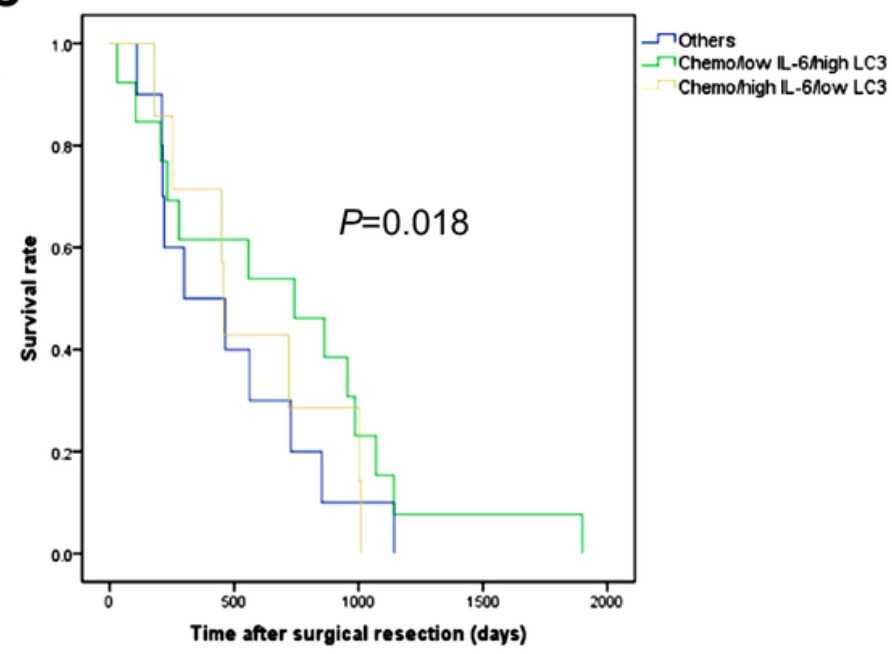

Figure 4

The Kaplan-Meier curves for overall survival rates according to adjuvant chemotherapy status, IL-6 in fibrotic area, and LC3 and p62 expression in cancer. The 70 patients were categorized based on having been or not subjected to chemotherapy and the respective level of expression of II-6, LC3 and p62 as detailed in Fig. S1 (see also the text for details). a It is apparent that the patients who received adjuvant chemotherapy and bearing a CCA with protein pattern of low IL-6, high LC3, and low p62 (L/H/L) expression (purple line) had an overall survival rate much better than any other combination; $P=0.01$. $b$ and $\mathrm{c}$ In the cohort of 30 patients subjected to chemotherapy, the group bearing a CCA with high LC3 and low stromal IL-6 (regardless of p62 expression) showed a better prognosis respect to the group with other combinations (panel $b$, green line vs blue line; $P=0.001$, log-rank test) and to the groups bearing a tumor with high stromal IL-6 and low cancer cell LC3 (panel c, green line vs yellow line; $P=0.018$, log-rank test ) or a tumor with any other combination (panel c, green line vs blue line; $P=0.018$, log-rank test). 
A

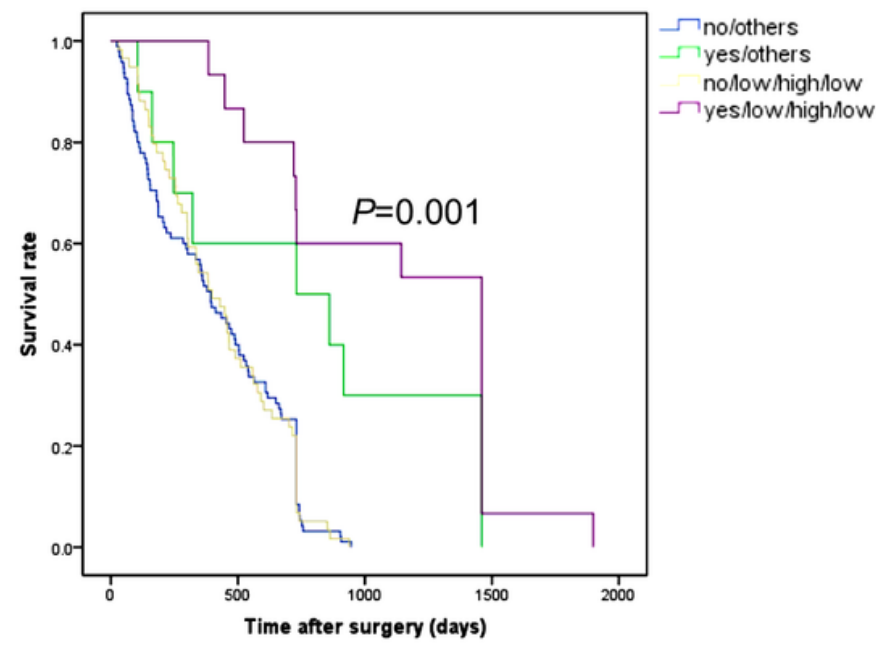

B

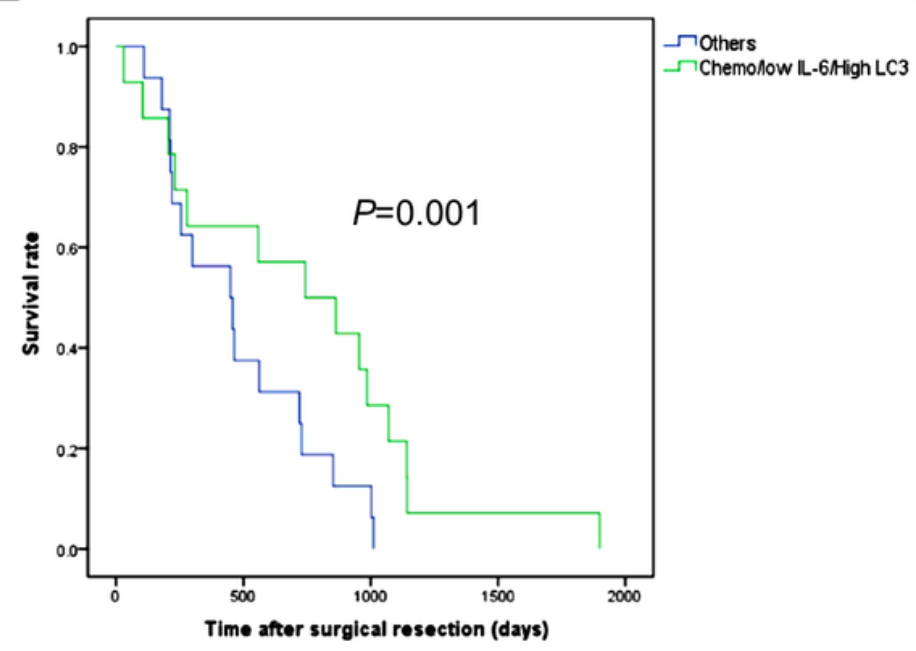

C

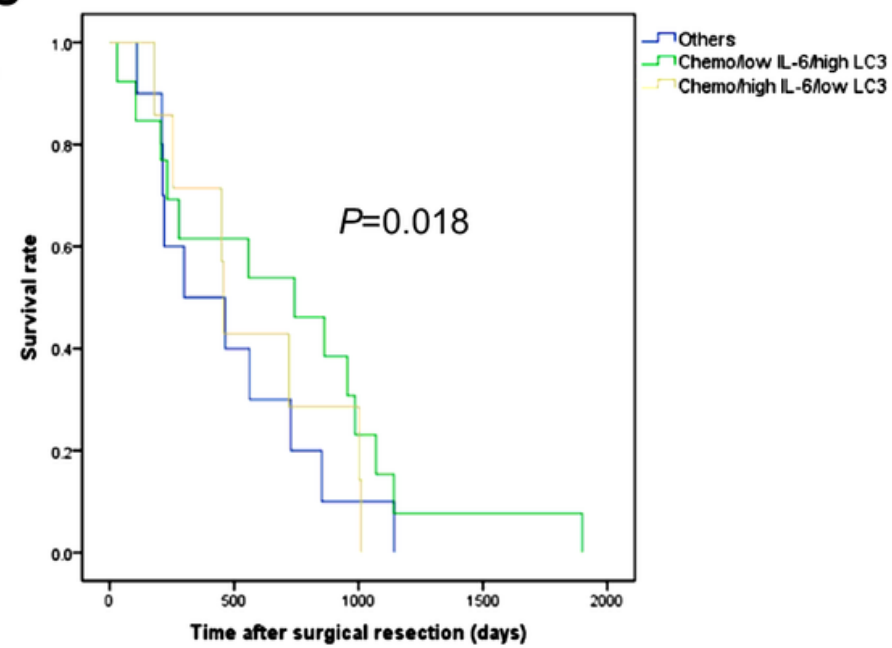

Figure 4

The Kaplan-Meier curves for overall survival rates according to adjuvant chemotherapy status, IL-6 in fibrotic area, and LC3 and p62 expression in cancer. The 70 patients were categorized based on having been or not subjected to chemotherapy and the respective level of expression of II-6, LC3 and p62 as detailed in Fig. S1 (see also the text for details). a It is apparent that the patients who received adjuvant chemotherapy and bearing a CCA with protein pattern of low IL-6, high LC3, and low p62 (L/H/L) expression (purple line) had an overall survival rate much better than any other combination; $P=0.01$. $b$ and $\mathrm{c}$ In the cohort of 30 patients subjected to chemotherapy, the group bearing a CCA with high LC3 and low stromal IL-6 (regardless of p62 expression) showed a better prognosis respect to the group with other combinations (panel $b$, green line vs blue line; $P=0.001$, log-rank test) and to the groups bearing a tumor with high stromal IL-6 and low cancer cell LC3 (panel c, green line vs yellow line; $P=0.018$, log-rank test ) or a tumor with any other combination (panel c, green line vs blue line; $P=0.018$, log-rank test). 
A

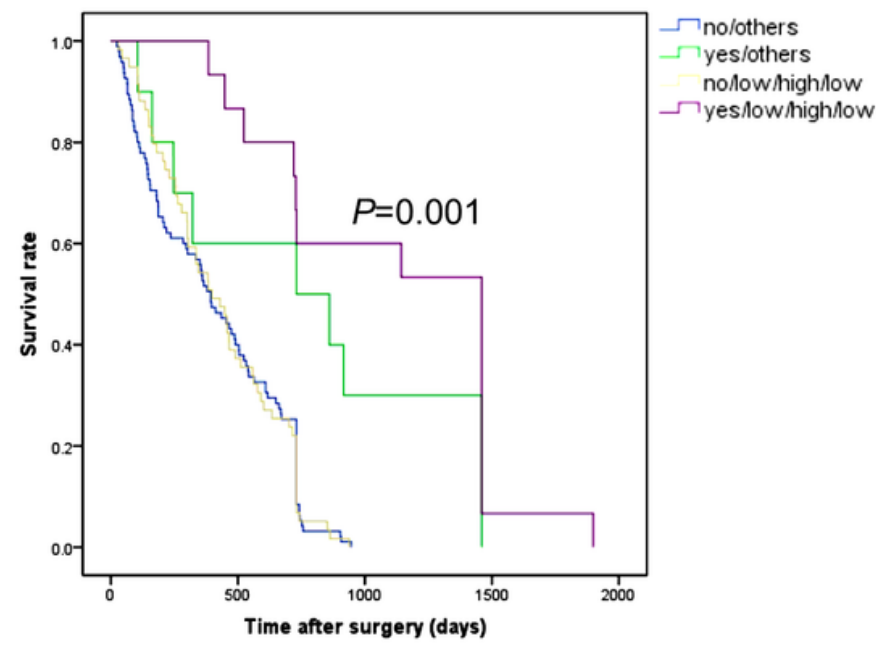

B

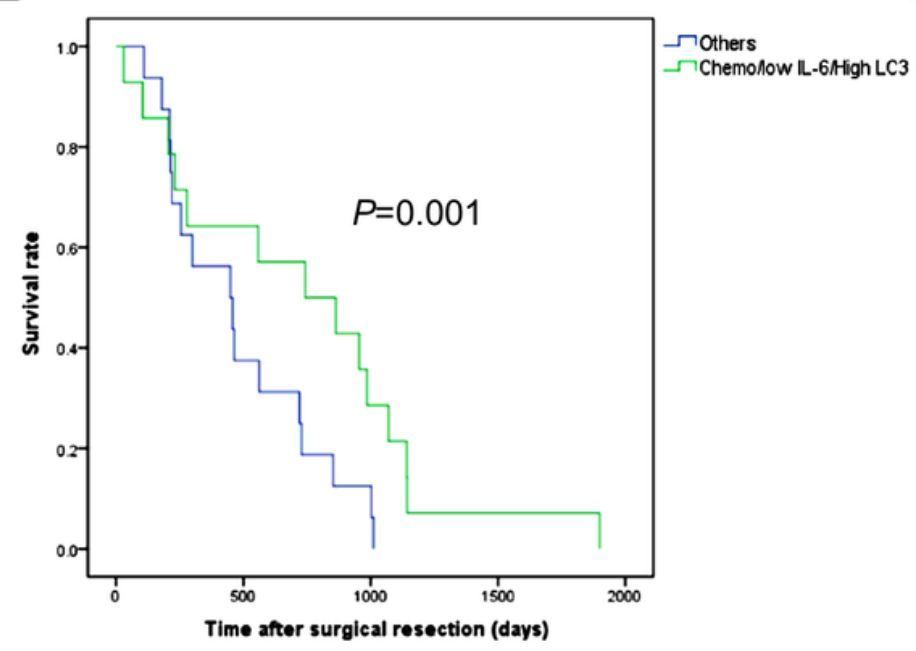

C

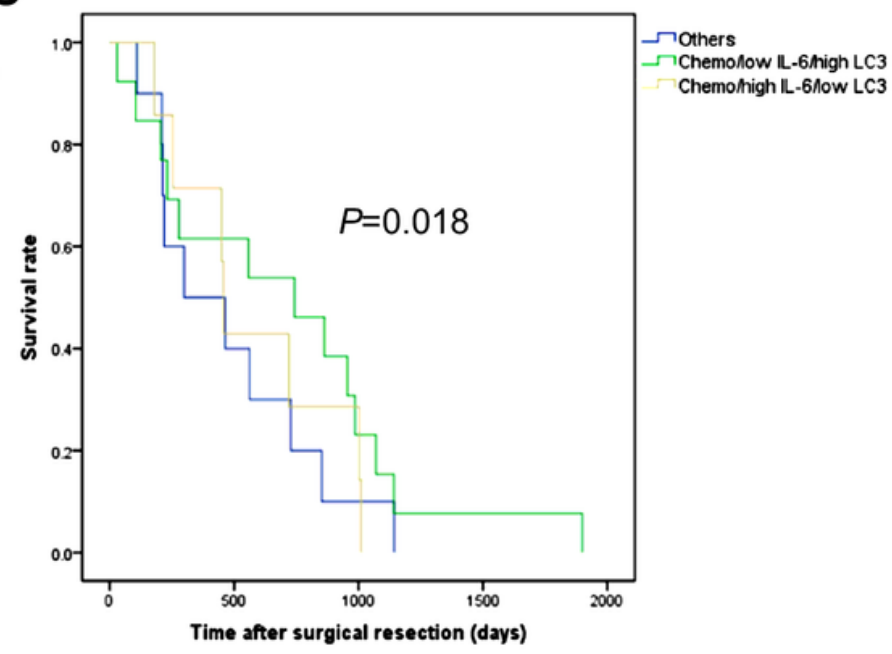

Figure 4

The Kaplan-Meier curves for overall survival rates according to adjuvant chemotherapy status, IL-6 in fibrotic area, and LC3 and p62 expression in cancer. The 70 patients were categorized based on having been or not subjected to chemotherapy and the respective level of expression of II-6, LC3 and p62 as detailed in Fig. S1 (see also the text for details). a It is apparent that the patients who received adjuvant chemotherapy and bearing a CCA with protein pattern of low IL-6, high LC3, and low p62 (L/H/L) expression (purple line) had an overall survival rate much better than any other combination; $P=0.01$. $b$ and $\mathrm{c}$ In the cohort of 30 patients subjected to chemotherapy, the group bearing a CCA with high LC3 and low stromal IL-6 (regardless of p62 expression) showed a better prognosis respect to the group with other combinations (panel $b$, green line vs blue line; $P=0.001$, log-rank test) and to the groups bearing a tumor with high stromal IL-6 and low cancer cell LC3 (panel c, green line vs yellow line; $P=0.018$, log-rank test ) or a tumor with any other combination (panel c, green line vs blue line; $P=0.018$, log-rank test). 
A

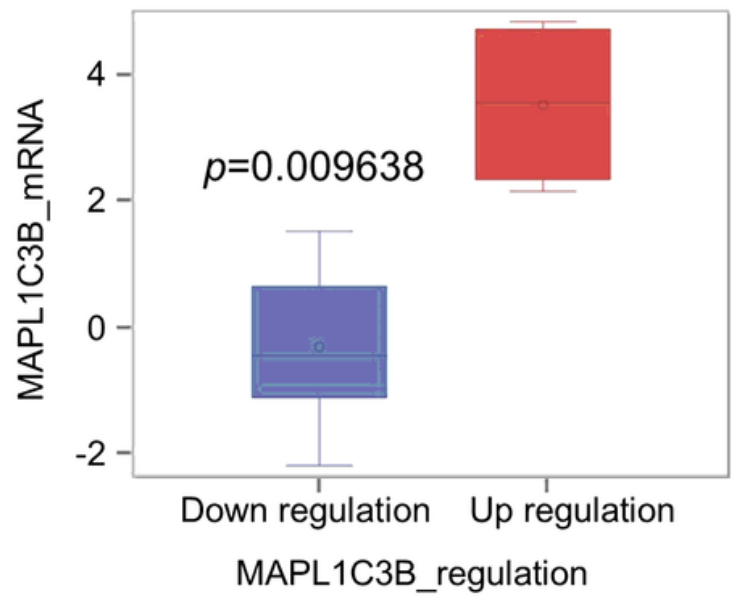

B

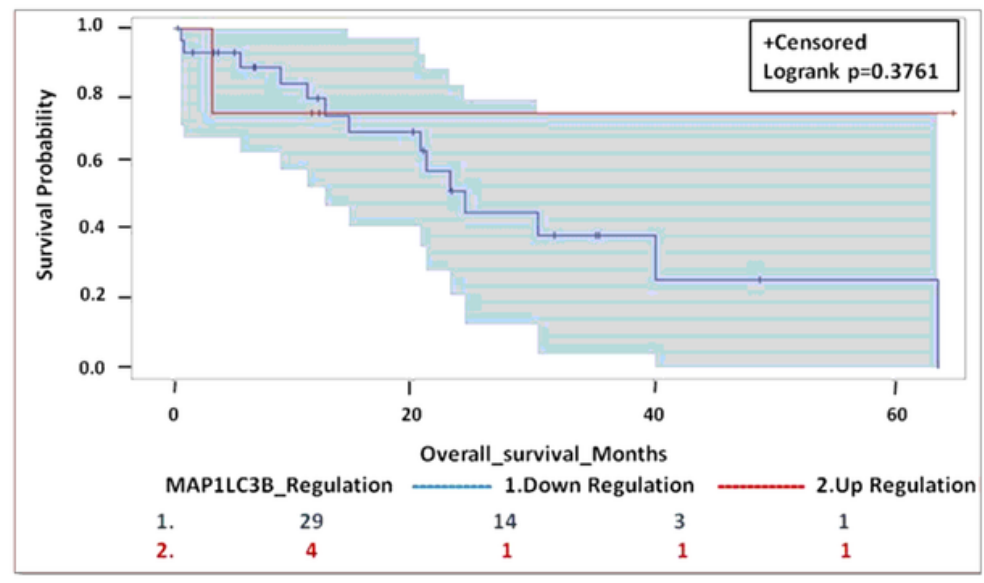

\section{Figure 5}

The prognostic value of autophagy marker LC3 mRNA in a cohort of CCA patients in the TCGA database. a Categorization of the CCA based on the level of MAP1LC3B (LC3) mRNA expression. b Patients bearing a CCA expressing high level of MAP1LC3B tend to have a longer overall survival $(P=0.3761)$.

A

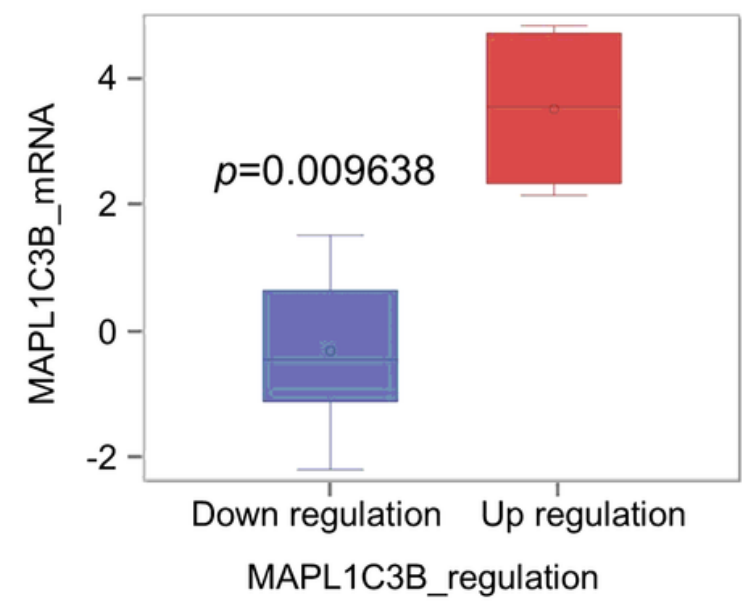

B

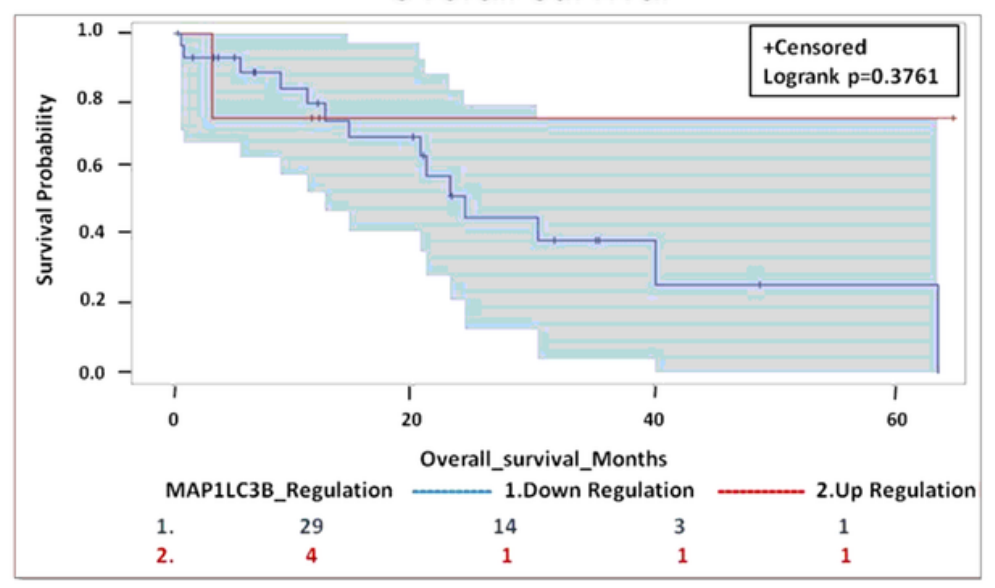

Figure 5

The prognostic value of autophagy marker LC3 mRNA in a cohort of CCA patients in the TCGA database. a Categorization of the CCA based on the level of MAP1LC3B (LC3) mRNA expression. b Patients bearing a CCA expressing high level of MAP1LC3B tend to have a longer overall survival $(P=0.3761)$. 
A

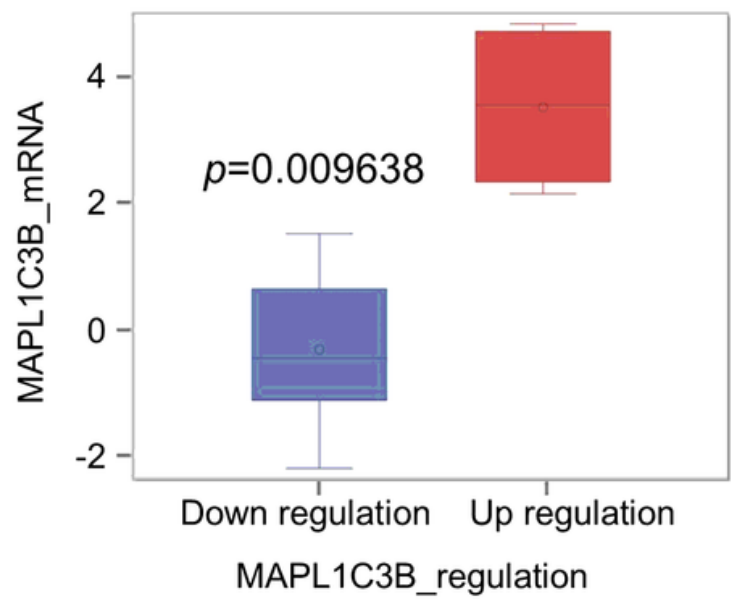

B

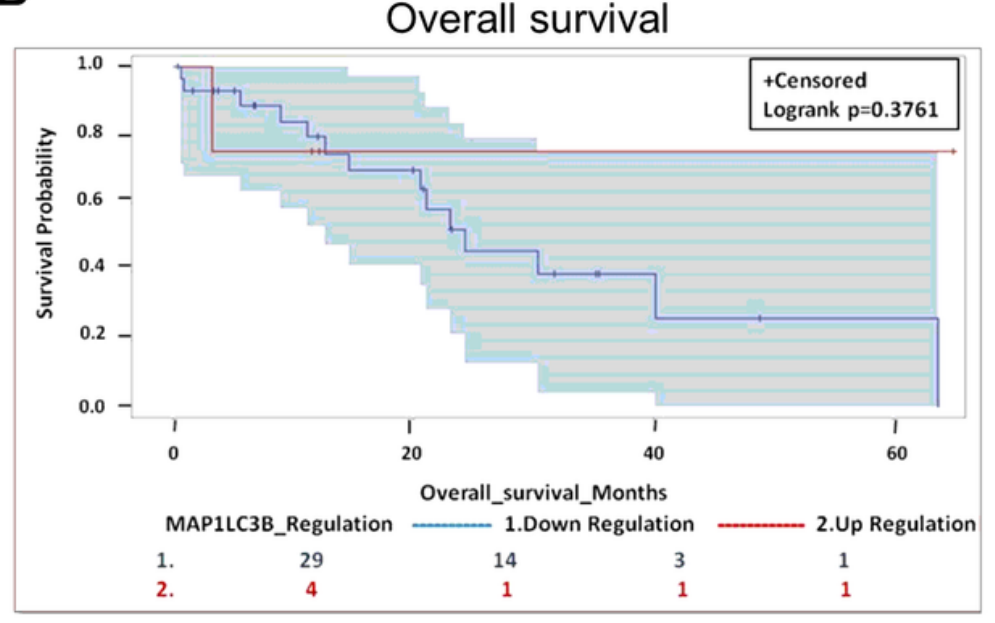

\section{Figure 5}

The prognostic value of autophagy marker LC3 mRNA in a cohort of CCA patients in the TCGA database. a Categorization of the CCA based on the level of MAP1LC3B (LC3) mRNA expression. b Patients bearing a CCA expressing high level of MAP1LC3B tend to have a longer overall survival $(P=0.3761)$. 
A

a-SMA

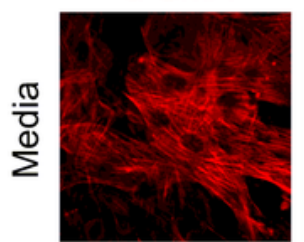

IL-6

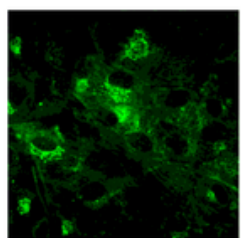

DAPI Merge
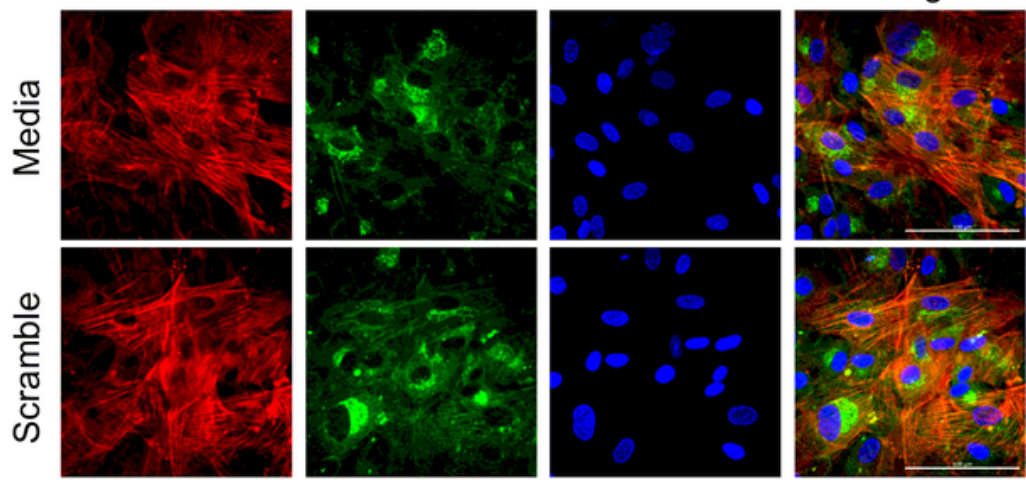

$\frac{1}{\frac{1}{\infty}}$
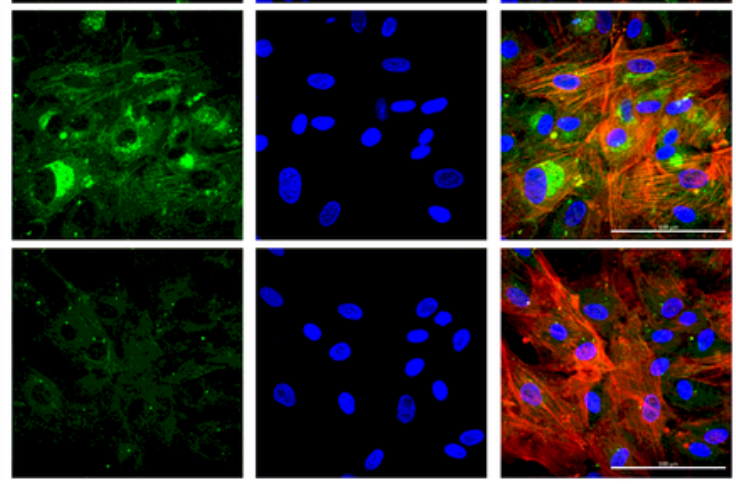

B

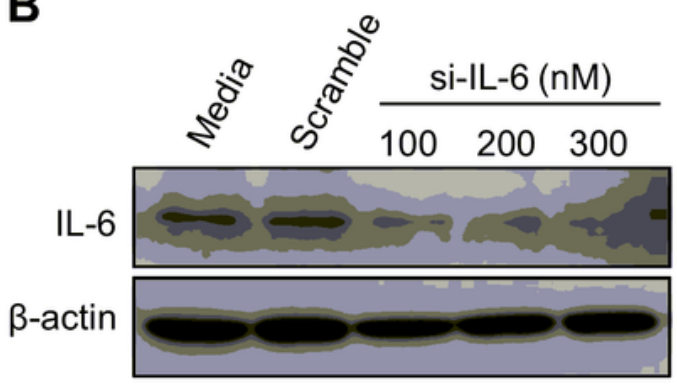

C $\quad 0.6$

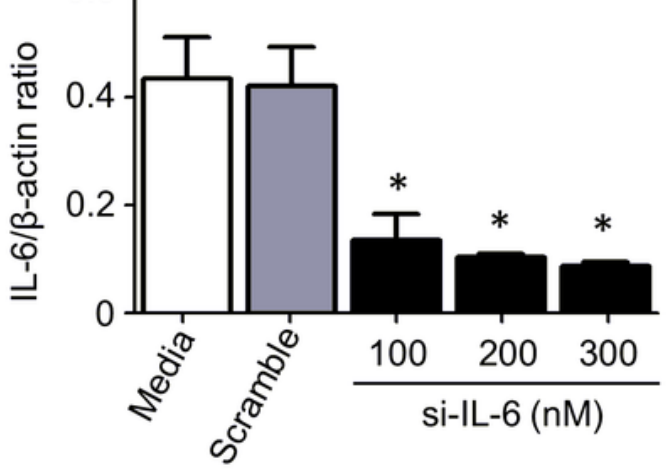

D

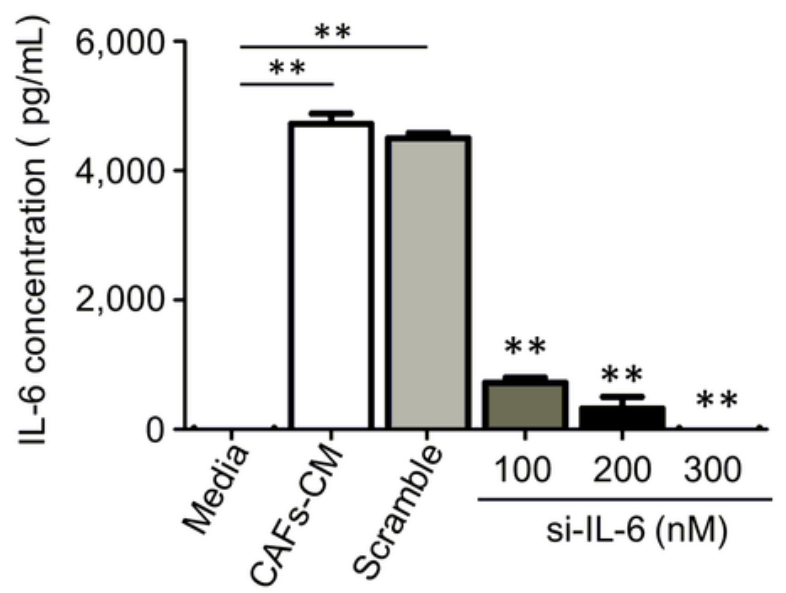

\section{Figure 6}

Efficacy of post-translational silencing of IL-6 in CAFs. Primary CAFs isolated from fresh CCA biopsies were transiently transfected with an IL-6 specific small interfering (si-) RNA and the efficacy of protein knockdown was tested by several methods including immunofluorescence (a), western blotting (b) and ELISA (c). a The cells were stained for alpha-SMA (red fluorescence; marker of CAFs), IL-6 (green fluorescence), and Hoechst (blue fluorescence; nuclei label). It is shown that si-RNA transfected CAFs 
express very low level of IL-6. (Magnification 63X). b CAFs were treated with control medium, scramble siRNA, or 100, 200, or $300 \mathrm{nM}$ of IL-6-specific si-RNA. The Western blotting shows a si-RNA dose-dependent reduction of cellular IL-6. The filter was stripped and re-probes for $\beta$-actin to verify protein loading. Densitometry is shown in c. d ELISA kit was used to analyze the levels of IL-6 in CAFs-CM after (or not) treatment with scramble or IL- 6 specific si-RNA. Values correspond to means $\pm S D$ of triplicates obtained in three independent experiments. ${ }^{\star} P<0.05$, ${ }^{\star *} P<0.01$, compared with scramble.
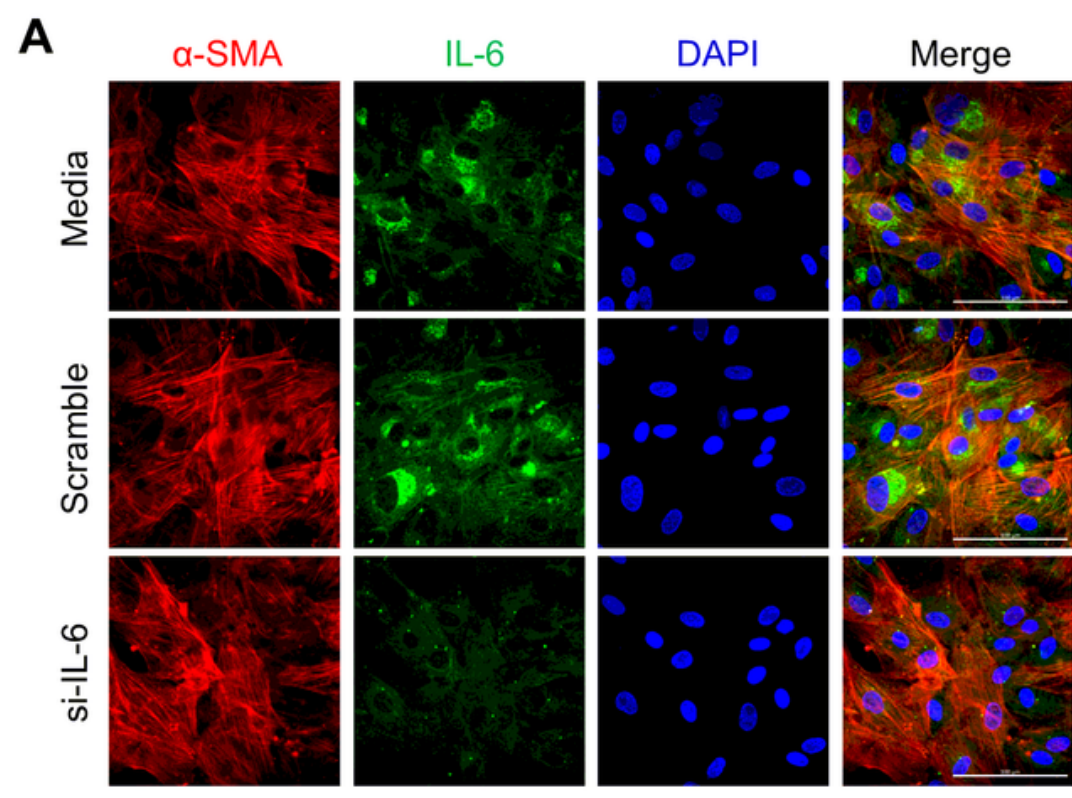

B
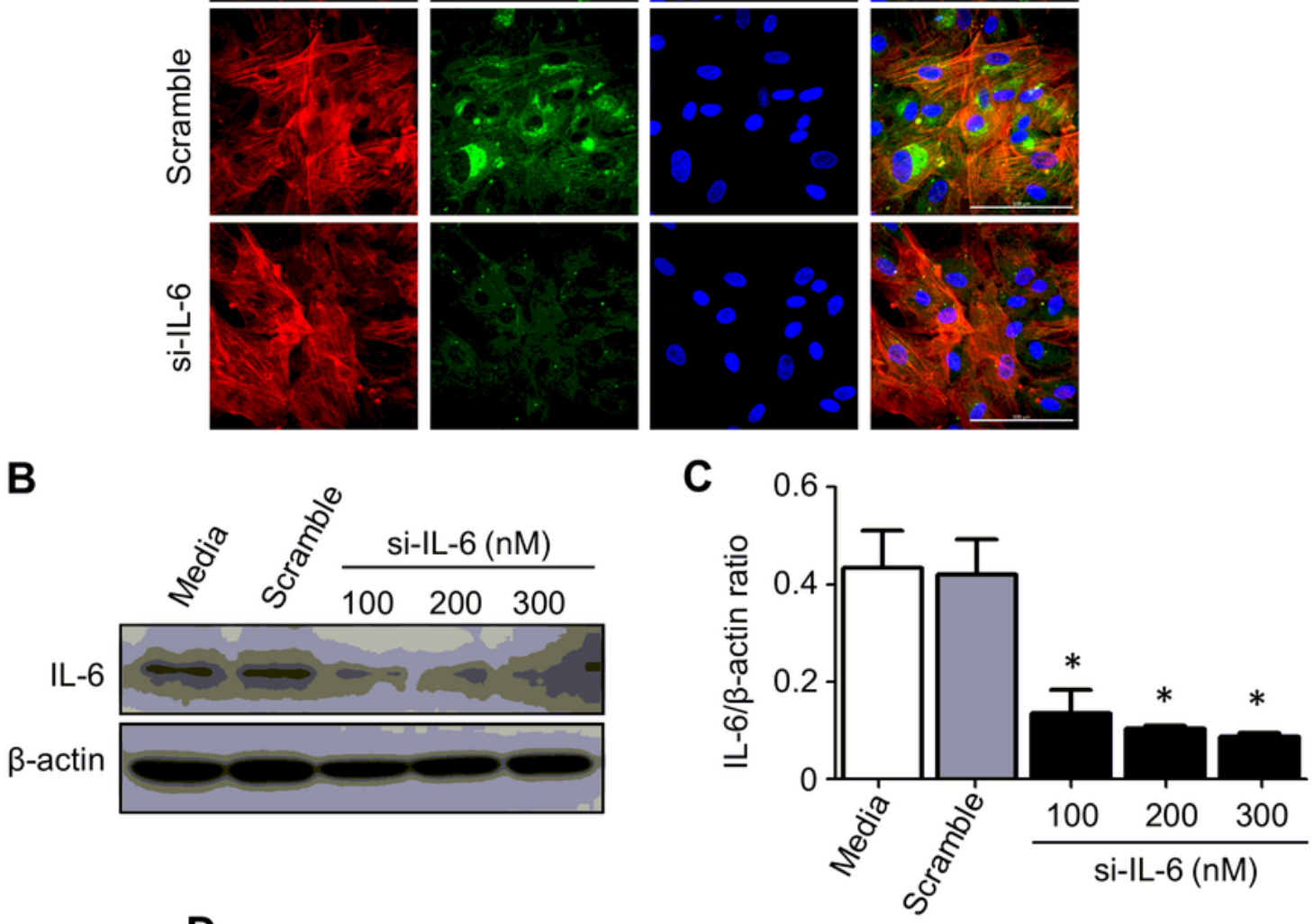

D

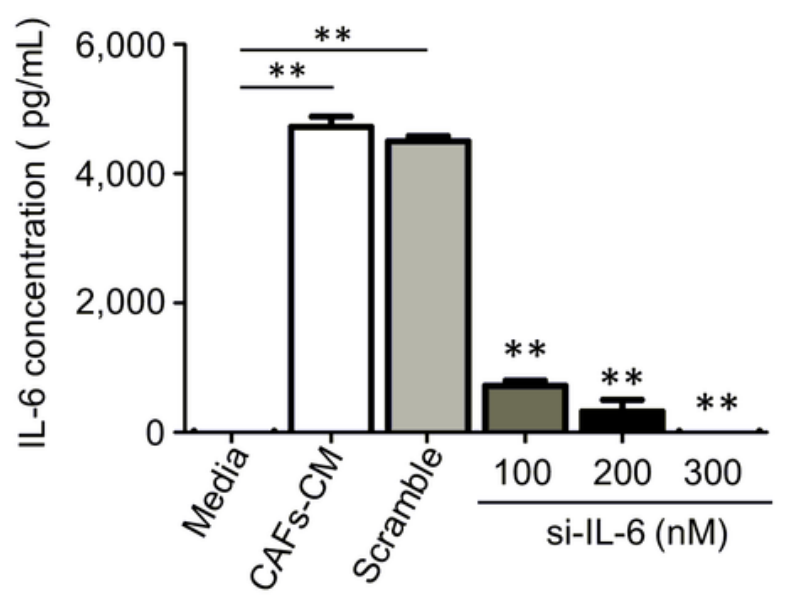

Figure 6 
Efficacy of post-translational silencing of IL- 6 in CAFs. Primary CAFs isolated from fresh CCA biopsies were transiently transfected with an IL-6 specific small interfering (si-) RNA and the efficacy of protein knockdown was tested by several methods including immunofluorescence (a), western blotting (b) and ELISA (c). a The cells were stained for alpha-SMA (red fluorescence; marker of CAFs), IL-6 (green fluorescence), and Hoechst (blue fluorescence; nuclei label). It is shown that si-RNA transfected CAFs express very low level of IL-6. (Magnification 63X). b CAFs were treated with control medium, scramble siRNA, or 100, 200, or $300 \mathrm{nM}$ of IL-6-specific si-RNA. The Western blotting shows a si-RNA dose-dependent reduction of cellular IL-6. The filter was stripped and re-probes for $\beta$-actin to verify protein loading. Densitometry is shown in c. $d$ ELISA kit was used to analyze the levels of IL-6 in CAFs-CM after (or not) treatment with scramble or IL- 6 specific si-RNA. Values correspond to means \pm SD of triplicates obtained in three independent experiments. ${ }^{\star} P<0.05$, ${ }^{\star} \mathrm{P}<0.01$, compared with scramble. 
A

a-SMA

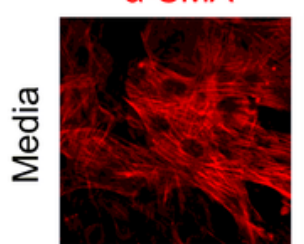

IL-6

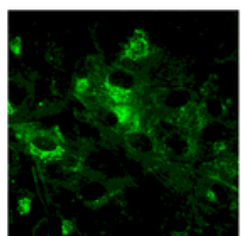

DAPI

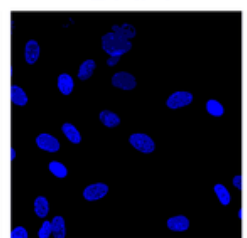

Merge
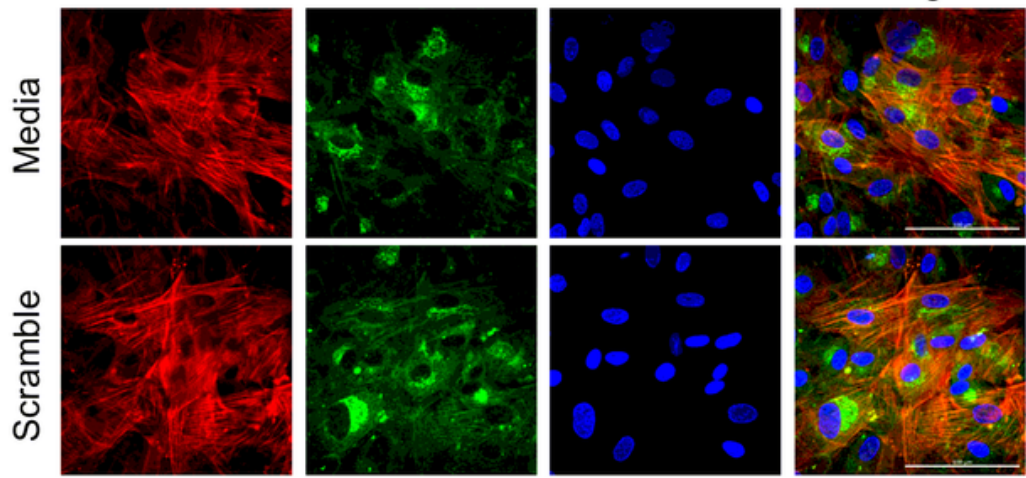

$\frac{1}{\frac{1}{4}}$
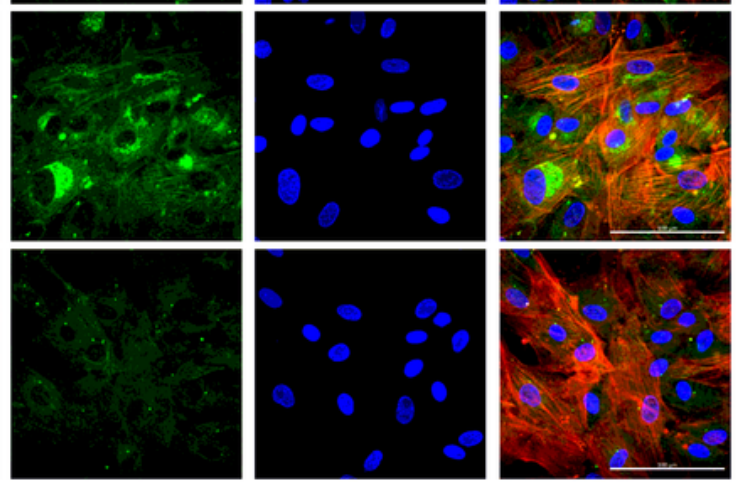

B

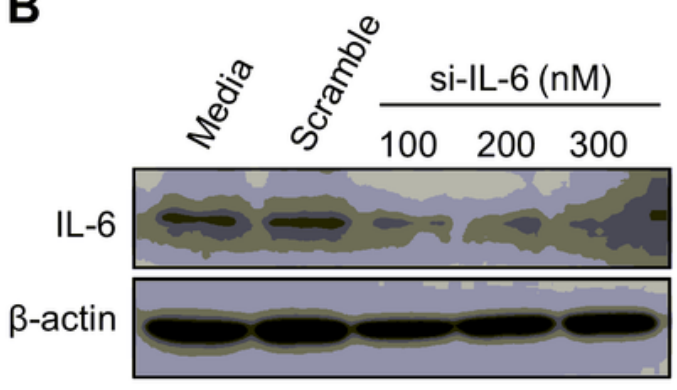

C $\quad 0.6$

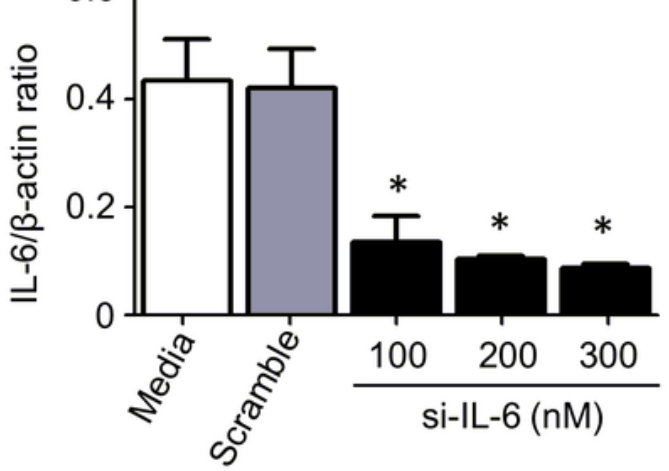

D

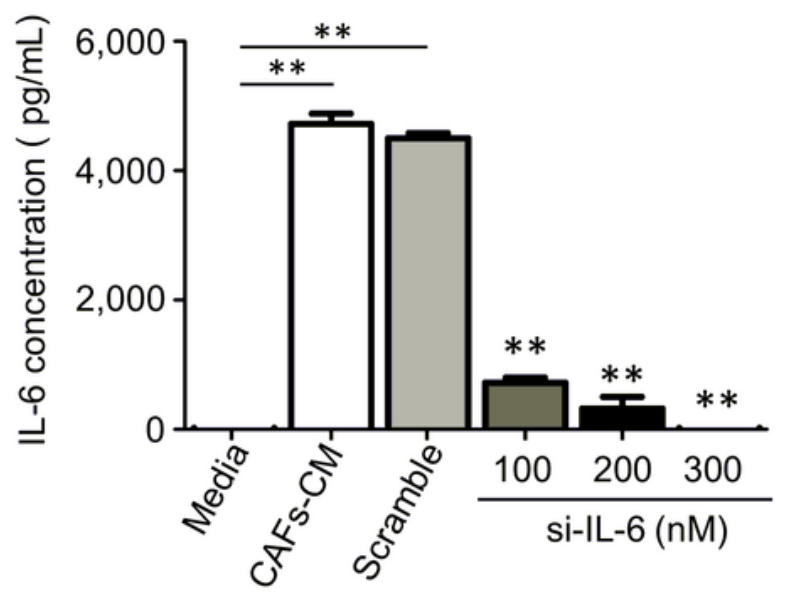

\section{Figure 6}

Efficacy of post-translational silencing of IL-6 in CAFs. Primary CAFs isolated from fresh CCA biopsies were transiently transfected with an IL-6 specific small interfering (si-) RNA and the efficacy of protein knockdown was tested by several methods including immunofluorescence (a), western blotting (b) and ELISA (c). a The cells were stained for alpha-SMA (red fluorescence; marker of CAFs), IL-6 (green fluorescence), and Hoechst (blue fluorescence; nuclei label). It is shown that si-RNA transfected CAFs 
express very low level of IL-6. (Magnification 63X). b CAFs were treated with control medium, scramble siRNA, or 100, 200, or 300 nM of IL-6-specific si-RNA. The Western blotting shows a si-RNA dose-dependent reduction of cellular IL-6. The filter was stripped and re-probes for $\beta$-actin to verify protein loading. Densitometry is shown in c. d ELISA kit was used to analyze the levels of IL-6 in CAFs-CM after (or not) treatment with scramble or IL- 6 specific si-RNA. Values correspond to means \pm SD of triplicates obtained in three independent experiments. ${ }^{\star} P<0.05,{ }^{\star *} P<0.01$, compared with scramble.

A

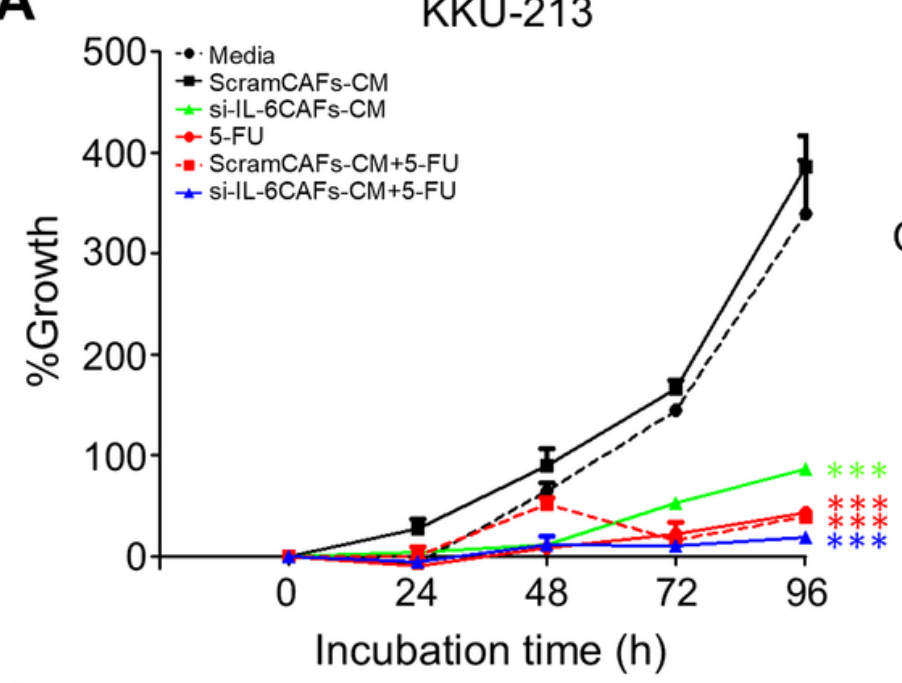

C

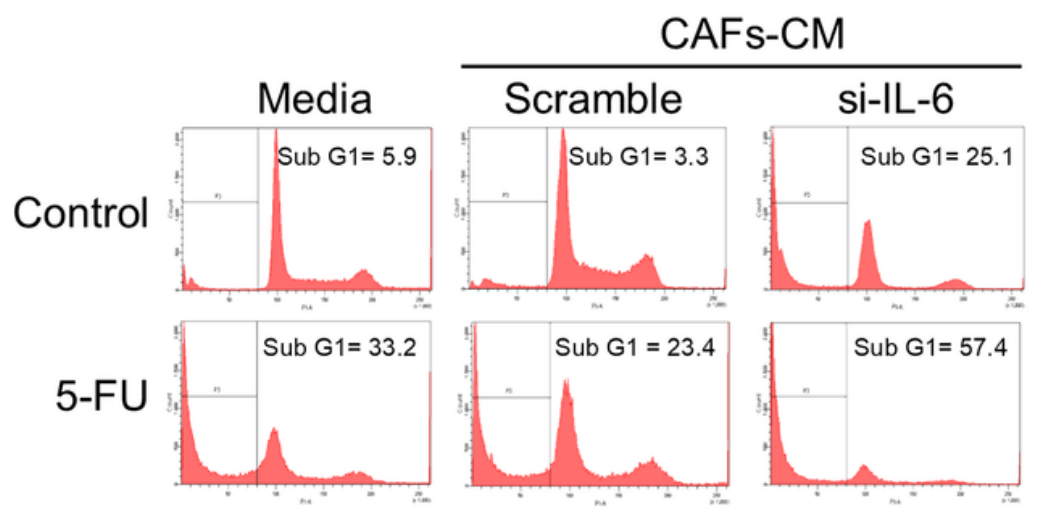

B

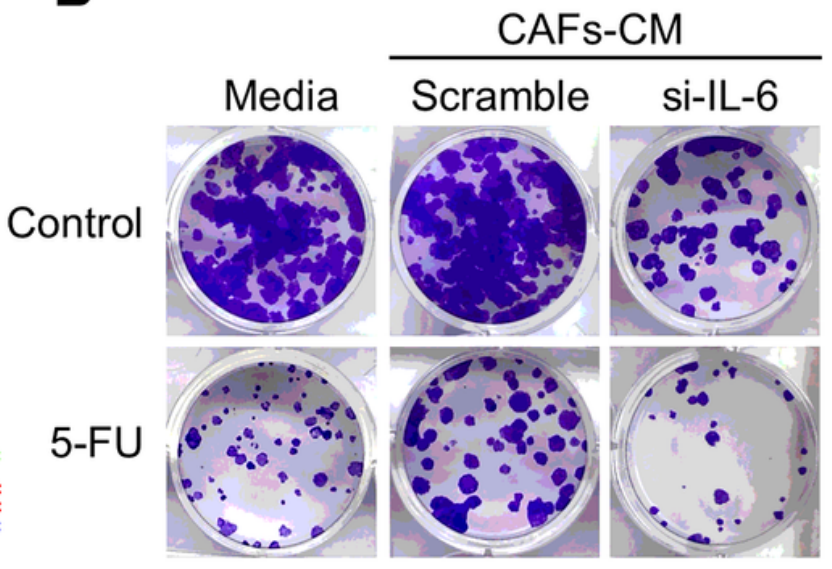

D

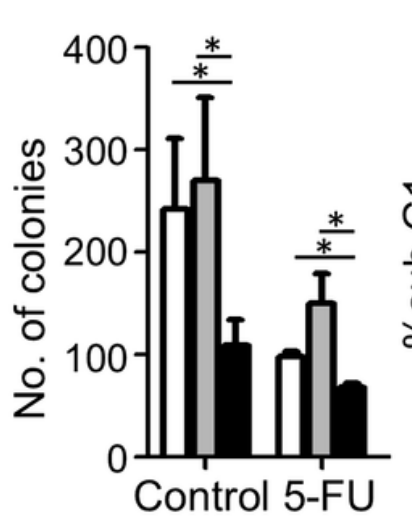

E
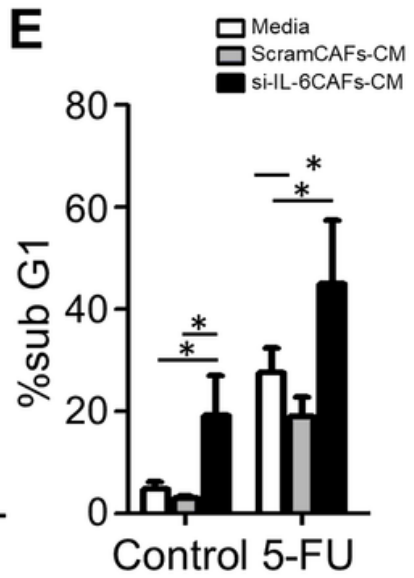

\section{Figure 7}

Effect of CAFs medium containing or not IL- 6 on chemosensitivity of CCA cells. KKU-213 cells were incubated in control medium or in conditioned medium (CM) from CAFs previously transfected with scramble or IL-6 specific si-RNA and then exposed or not to the chemotherapeutic drug 5-FU, as indicated in the panels. a Cell survival as determined by SRB assay after $48 \mathrm{~h}$ of culture. $\mathrm{b}$ Clonogenic assay and crystal violet staining. c Cytofluorometric analysis of the sub G1 population after staining with propidium iodide $(\mathrm{PI})$ of the cells treated as indicated. Data represent the mean \pm S.D. of at least three different experiments run in triplicate. ${ }^{*} \mathrm{P}<0.05$ compared to control or medium alone. 
A

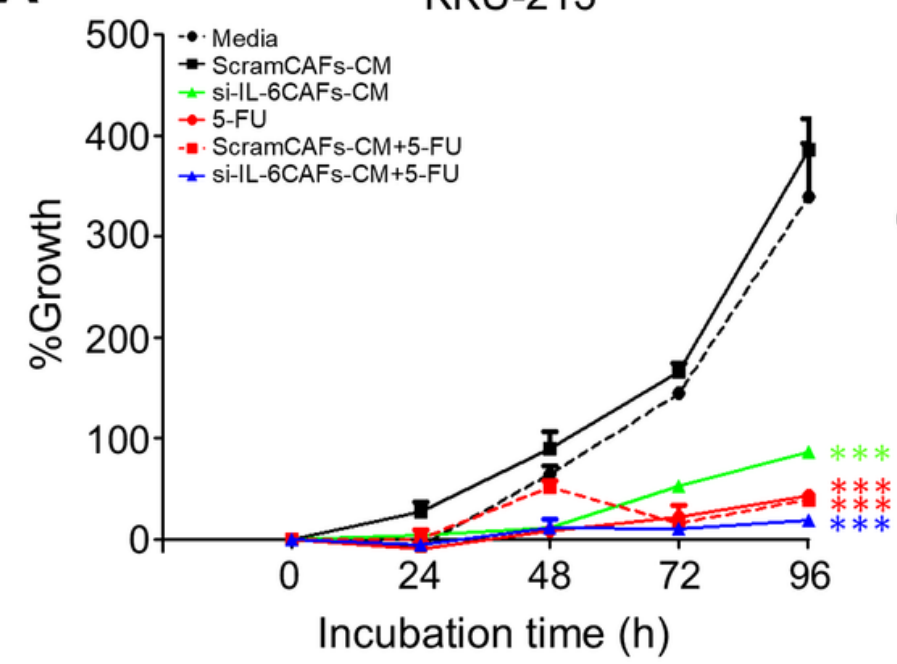

C

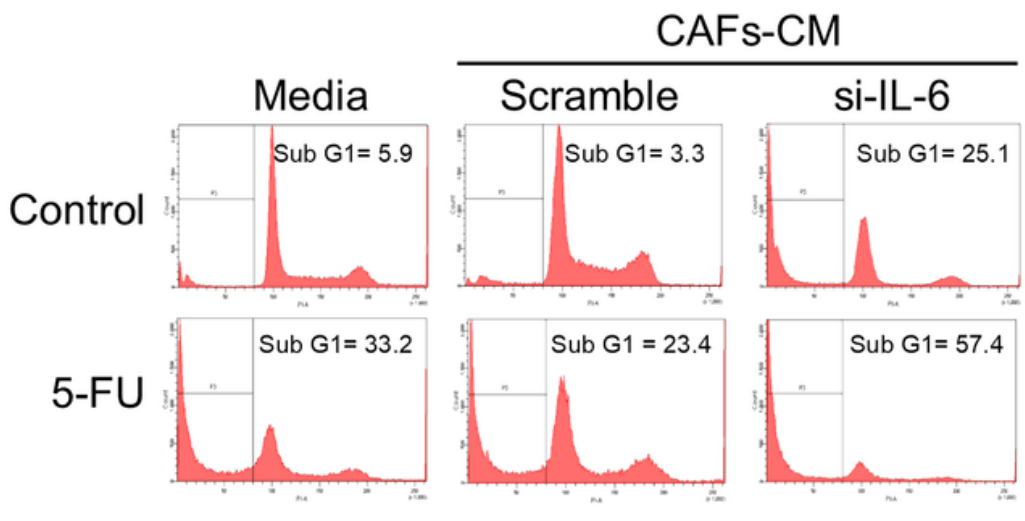

B

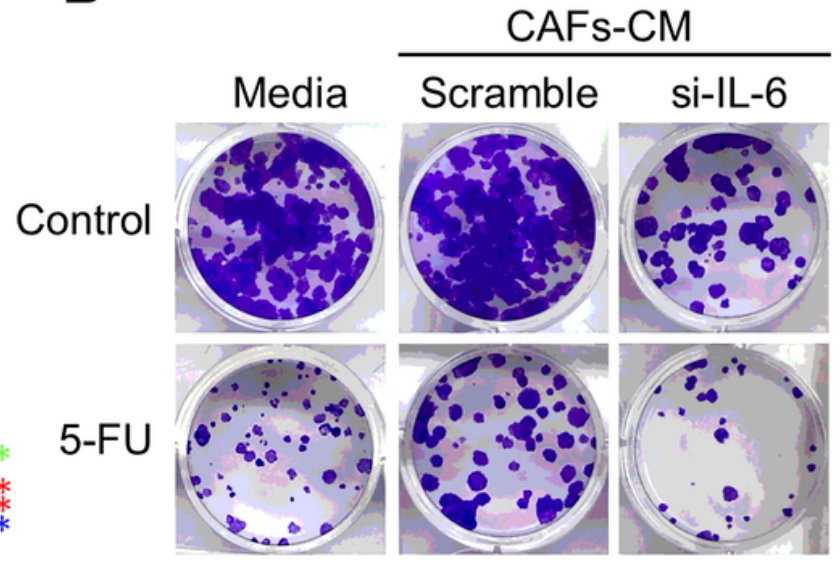

D

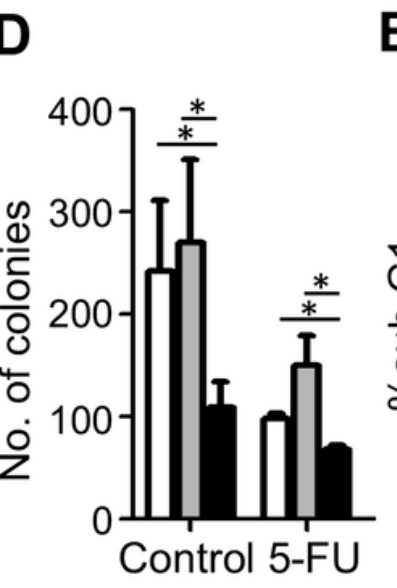

E $\quad$ 口edia $80 \operatorname{Q}_{\text {sill-6CAFs-CM }}^{\text {ScramCAFs-CM }}$

Figure 7

Effect of CAFs medium containing or not IL-6 on chemosensitivity of CCA cells. KKU-213 cells were incubated in control medium or in conditioned medium (CM) from CAFs previously transfected with scramble or IL-6 specific si-RNA and then exposed or not to the chemotherapeutic drug 5-FU, as indicated in the panels. a Cell survival as determined by SRB assay after $48 \mathrm{~h}$ of culture. $\mathrm{b}$ Clonogenic assay and crystal violet staining. c Cytofluorometric analysis of the sub G1 population after staining with propidium iodide $(\mathrm{PI})$ of the cells treated as indicated. Data represent the mean \pm S.D. of at least three different experiments run in triplicate. ${ }^{*} \mathrm{P}<0.05$ compared to control or medium alone. 
A

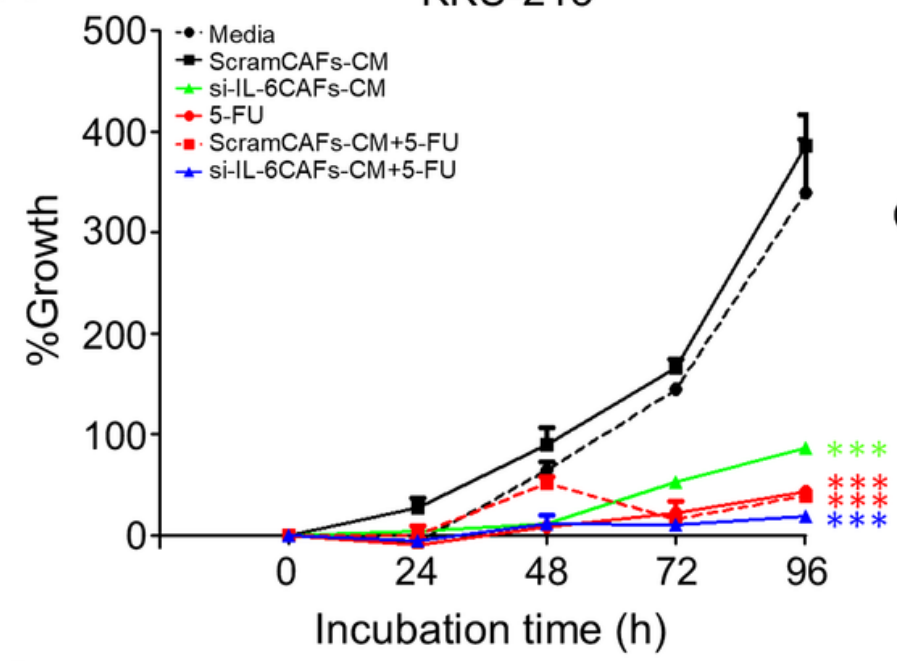

C

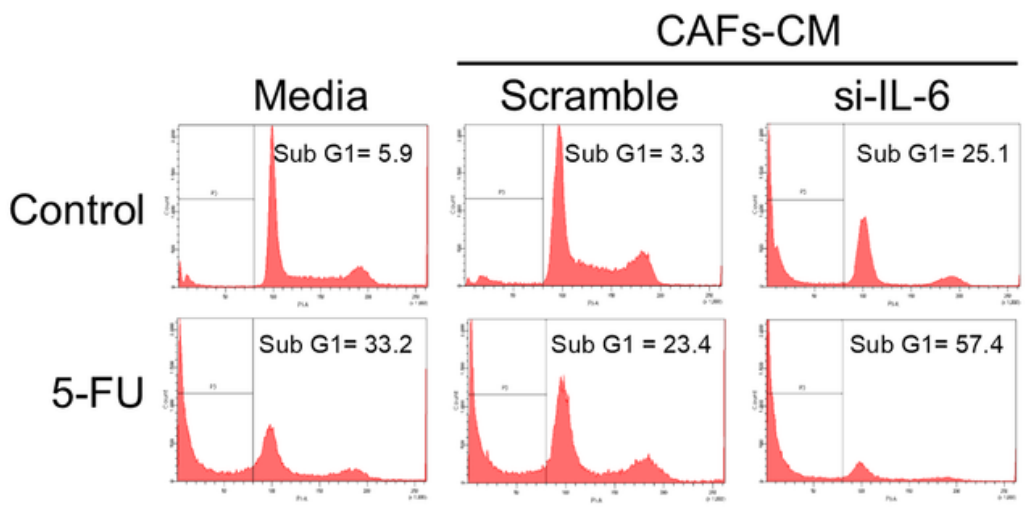

B

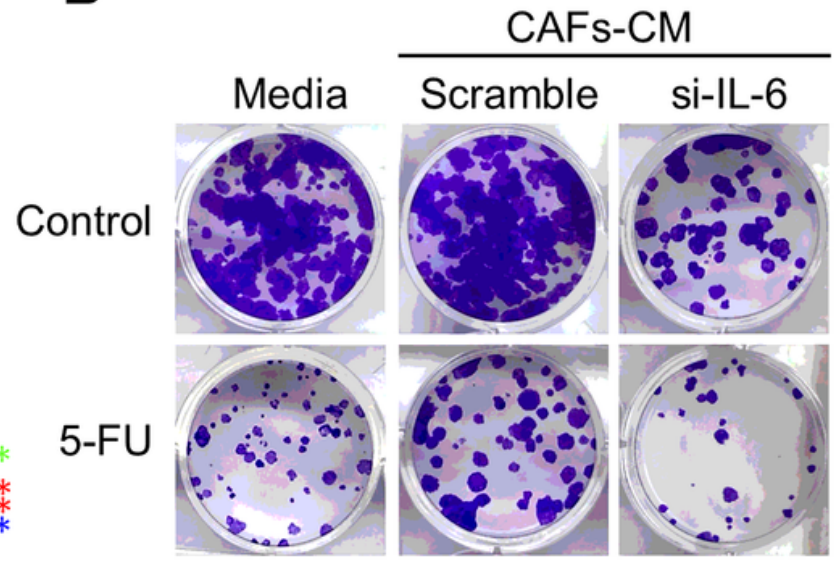

D

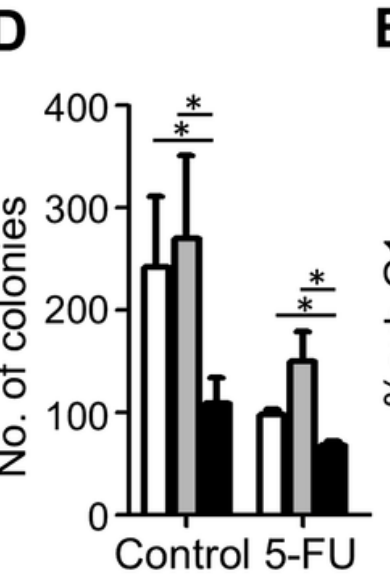

E 807 Di-lL-6CAFs-CM

Figure 7

Effect of CAFs medium containing or not IL-6 on chemosensitivity of CCA cells. KKU-213 cells were incubated in control medium or in conditioned medium (CM) from CAFs previously transfected with scramble or IL-6 specific si-RNA and then exposed or not to the chemotherapeutic drug 5-FU, as indicated in the panels. a Cell survival as determined by SRB assay after $48 \mathrm{~h}$ of culture. $\mathrm{b}$ Clonogenic assay and crystal violet staining. c Cytofluorometric analysis of the sub G1 population after staining with propidium iodide $(\mathrm{PI})$ of the cells treated as indicated. Data represent the mean \pm S.D. of at least three different experiments run in triplicate. ${ }^{*} \mathrm{P}<0.05$ compared to control or medium alone. 
A

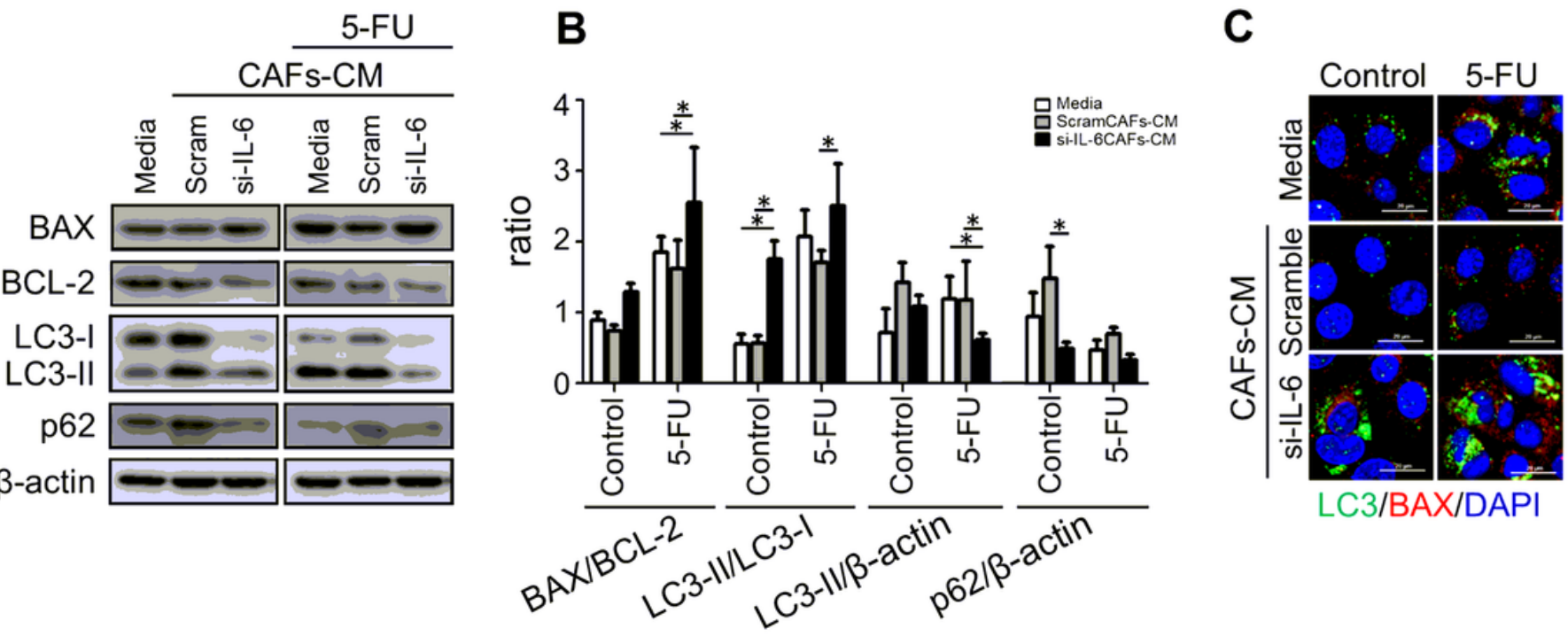

Figure 8

Expression apoptosis- and of autophagy-related proteins in KKU-213 cells exposed to CAFs medium and 5 -FU. KKU cells were incubated in control or conditioned medium (CM) from CAFs previously transfected with scramble or IL-6 specific si-RNA and then exposed or not for $48 \mathrm{~h}$ to the chemotherapeutic drug 5-FU. At the end, expression of relevant proteins involved in apoptosis (BAX and BCL-2) and autophagy (LC3 and p62) were determined by western blotting (panel a) or immunofluorescence (panel c; Scale bar=20 $\mu \mathrm{M}$; magnification 63X). Western blotting and immunofluorescence images are representative of three independent experiments. Densitometry of western blotting data are reported as mean \pm SD in panel $B$. Statistical significance * $\mathrm{P}<0.05$ compared to control.
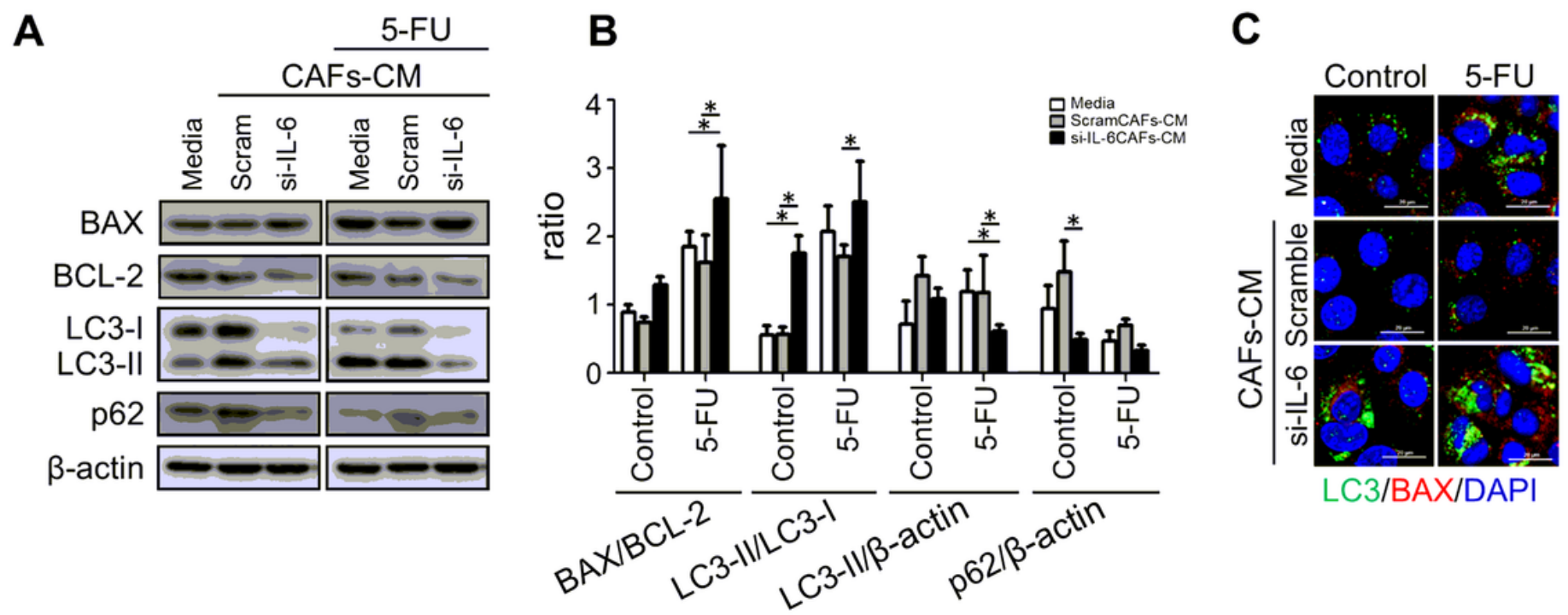

Figure 8 
Expression apoptosis- and of autophagy-related proteins in KKU-213 cells exposed to CAFs medium and 5 -FU. KKU cells were incubated in control or conditioned medium (CM) from CAFs previously transfected with scramble or IL-6 specific si-RNA and then exposed or not for $48 \mathrm{~h}$ to the chemotherapeutic drug 5-FU. At the end, expression of relevant proteins involved in apoptosis (BAX and BCL-2) and autophagy (LC3 and p62) were determined by western blotting (panel a) or immunofluorescence (panel c; Scale bar=20 $\mu \mathrm{M}$; magnification $63 \mathrm{X}$ ). Western blotting and immunofluorescence images are representative of three independent experiments. Densitometry of western blotting data are reported as mean \pm SD in panel $B$. Statistical significance * $\mathrm{P}<0.05$ compared to control.

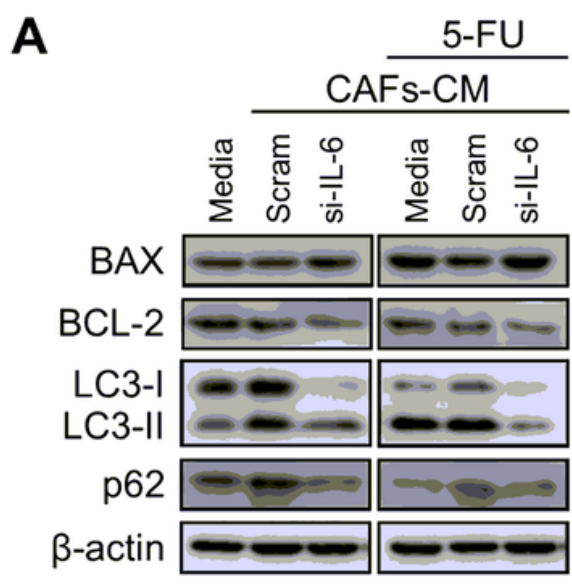

B

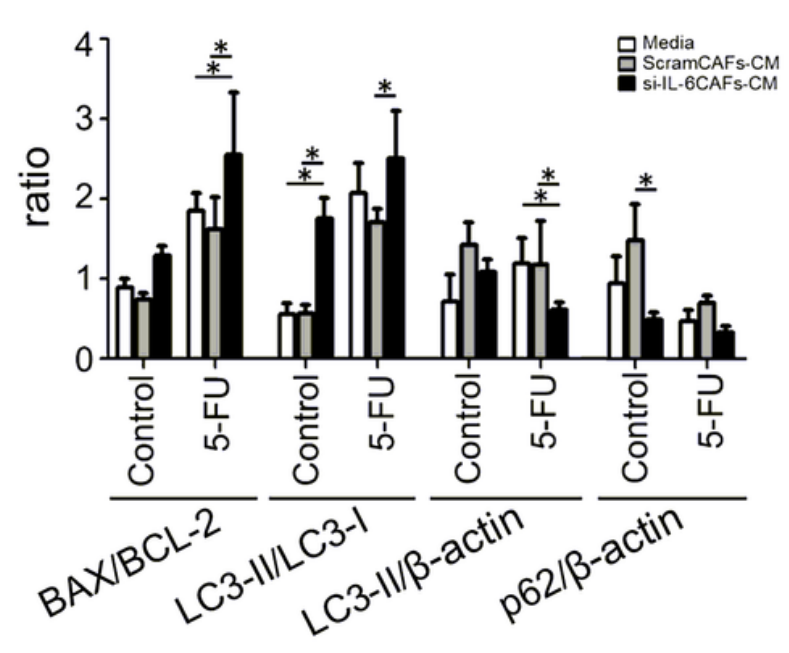

C

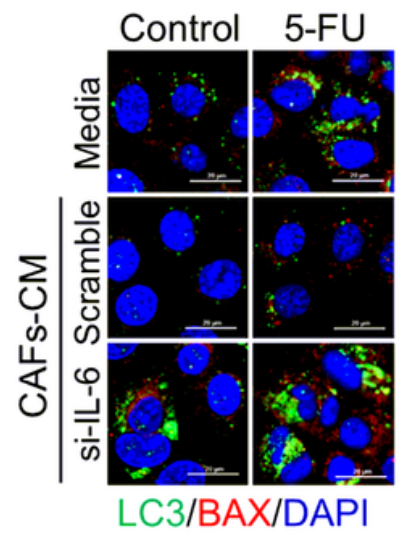

\section{Figure 8}

Expression apoptosis- and of autophagy-related proteins in KKU-213 cells exposed to CAFs medium and 5 -FU. KKU cells were incubated in control or conditioned medium (CM) from CAFs previously transfected with scramble or IL-6 specific si-RNA and then exposed or not for $48 \mathrm{~h}$ to the chemotherapeutic drug 5-FU. At the end, expression of relevant proteins involved in apoptosis (BAX and BCL-2) and autophagy (LC3 and p62) were determined by western blotting (panel a) or immunofluorescence (panel c; Scale bar=20 $\mu \mathrm{M}$; magnification 63X). Western blotting and immunofluorescence images are representative of three independent experiments. Densitometry of western blotting data are reported as mean \pm SD in panel $B$. Statistical significance * $\mathrm{P}<0.05$ compared to control.

\section{Supplementary Files}

This is a list of supplementary files associated with this preprint. Click to download.

- ThongchotSAdditionalfile1.docx

- ThongchotSAdditionalfile1.docx

- ThongchotSAdditionalfile1.docx 
- ThongchotSAdditionalfile2.docx

- ThongchotSAdditionalfile2.docx

- ThongchotSAdditionalfile2.docx 